\title{
A new hadron spectroscopy
}

\author{
Stephen Lars Olsen \\ Center for Underground Physics, Institute of Basic Science, Daejeon 305-811, Korea \\ E-mail: solensnu@gmail.com \\ Received August 13, 2014; accepted September 16, 2014
}

\begin{abstract}
QCD-motivated models for hadrons predict an assortment of "exotic" hadrons that have structures that are more complex than the quark-antiquark mesons and three-quark baryons of the original quark-parton model. These include pentaquark baryons, the six-quark $H$-dibaryon, and tetraquark, hybrid and glueball mesons. Despite extensive experimental searches, no unambiguous candidates for any of these exotic configurations have been identified. On the other hand, a number of meson states, one that seems to be a proton-antiproton bound state, and others that contain either charmedanticharmed quark pairs or bottom-antibottom quark pairs, have been recently discovered that neither fit into the quark-antiquark meson picture nor match the expected properties of the QCDinspired exotics. Here I briefly review results from a recent search for the $H$-dibaryon, and discuss some properties of the newly discovered states -the proton-antiproton state and the so-called $X Y Z$ mesons- and compare them with expectations for conventional quark-antiquark mesons and the predicted QCD-exotic states.
\end{abstract}

Keywords quarks, charmonium, pentaquarks, di-baryons, baryonium, tetraquarks

PACS numbers 14.40.Gx, 12.39.Mk, 13.20.He

\section{Contents}

1 Introduction

2 Pentaquarks and $H$-dibaryons

2.1 Belle $H$-dibaryon search

3 What we do see

3.1 Baryonium in radiative $J / \psi \rightarrow \gamma p \bar{p}$ decays?

3.2 The $X Y Z$ mesons

3.2.1 Charmoniumlike mesons

3.2.2 Bottomoniumlike mesons

3.3 Comments

3.3.1 Molecules?

3.3.2 Tetraquarks?

3.3.3 QCD-hybrids?

3.3.4 Hadrocharmonium?

3.3.5 A unified model?

4 Summary

Acknowledgements

References and notes

\section{Introduction}

The strongly interacting particles of the Standard Model are colored quarks and gluons. In contrast, the strongly interacting particles in nature are color-singlet (i.e., white) mesons and baryons. In the theory, quarks and gluons are related to mesons and baryons by the longdistance regime of Quantum Chromodynamics (QCD), which remains the least understood aspect of the theory. Since first-principle lattice-QCD (LQCD) calculations are still not practical for most long-distance phenomena, a number of models motivated by the color structure of QCD have been proposed. However, so far at least, predictions of these QCD-motivated models that pertain to the spectrum of hadrons have not had great success.

For example, it is well known that combining a $q=$ $u, d, s$ light-quark triplet with a $\bar{q}=\bar{u}, \bar{d}, \bar{s}$ antiquark antitriplet gives the familiar meson octet of flavor- $S U(3)$. Using similar considerations based on QCD, two quark triplets can be combined to form a "diquark" antitriplet of antisymmetric $q q$ states and a sextet of symmetric states as illustrated in Fig. 1(a). In QCD, these diquarks have color: combining a red triplet with a blue triplet - as shown in the figure - produces a magenta (anti-green) diquark and, for the antisymmetric triplet configurations, the color force between the two quarks is expected to be attractive. Likewise, green-red and blue-green diquarks form yellow (anti-blue) and cyan (anti-red) antitriplets as shown in Fig. 1(b).

Since these diquarks are not color-singlets, they cannot exist as free particles but, on the other hand, the 
anticolored diquark antitriplets should be able to combine with other colored objects in a manner similar to antiquark antitriplets, thereby forming multiquark colorsinglet states with a more complex substructure than the $q \bar{q}$ mesons and $q q q$ baryons of the original quark model [1]. These so-called "exotic" states include pentaquark baryons, six-quark $H$-dibaryons and tetraquark mesons, as illustrated in Fig. 1(c). Other proposed exotic states are glueballs, which are mesons made only from gluons, hybrids formed from a $q, \bar{q}$ and a gluon, and deuteronlike bound states of color-singlet "normal" hadrons, commonly referred to as molecules. These are illustrated in Fig. 1(d). Glueball and hybrid mesons are motivated by QCD; molecules are a generalization of classical nuclear physics to systems of subatomic partics.

(a)



(b)


(c)

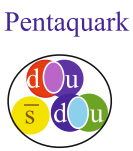

diquark-diquarkantiquark



diquark-diquarkdiquark

(d)

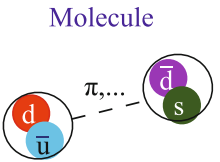

perimental investigations during the four decades that have elapsed since QCD was first formulated. This activity peaked in 2003 when the LEPS experiment at the SPring-8 electron ring in Japan reported the observation of a peak in the $K^{+} n$ invariant mass distribution in $\gamma n \rightarrow K^{+} K^{-} n$ reactions on a carbon target [2], with properties close to those that had been predicted for the $S=+1 \Theta_{5}^{+}$pentaquark [3]. This created a lot of excitement at the time [4] but subsequent, high-statistics experiments $[5,6]$ did not confirm the LEPS result and, instead, gave negative results. The current "conventional wisdom" is that pentaquarks do not exist [7], or at least have yet to be found.

The six-quark $H$-dibaryon, subsequently referred to as $H$, was predicted by Jaffee in 1977 to be a doubly strange, tightly bound six-quark structure (uuddss) with isospin zero and $J^{P}=0^{+}$[8]. An $S=-2$ state with baryon number $B=2$ and mass below $2 m_{\Lambda}$ could only decay via weak interactions and, thus, would be longlived. Although Jaffe's original prediction that the $H$ would be $\sim 80 \mathrm{MeV}$ below the $2 m_{\Lambda}$ threshold was ruled out by the observation of double- $\Lambda$ hypernuclei, most notably the famous "Nagara" event [9] that limited the allowed $H$ region to masses above $2 m_{\Lambda}-7.7 \mathrm{MeV}$, the theoretical case for an $H$-dibaryon with mass near $2 m_{\Lambda}$ continues to be strong, and has been recently strengthened by two independent LQCD calculations, both of which find an $H$-dibaryon state with mass near $2 m_{\Lambda}[10$, 11].

\subsection{Belle $H$-dibaryon search}

The Belle experiment recently reported results of a search for production of an $H$-dibaryon with mass near $2 m_{\Lambda}$ in inclusive $\Upsilon(1 S)$ and $\Upsilon(2 S)$ decays [12]. Decays of narrow $\Upsilon(n S)(n=1,2,3)$ bottomonium $(b \bar{b})$ resonances are particularly well suited for searches for multiquark states with non-zero strangeness. The $\Upsilon(n S)$ states are flavor- $S U(3)$ singlets that primarily decay via annihilation into three gluons. The gluons materialize into $u \bar{u}, d \bar{d}$ and $s \bar{s}$ pairs with nearly equal probabilities, plus additional gluons that also subsequently materialize as $q \bar{q}$ pairs. This creates final states with a high density of quarks and antiquarks in a limited volume of phase space. A benchmark rate for multiquark-state production in these decays is set by the measured inclusive decay branching fractions to antideuterons $(\bar{D})^{1)}$ : $\mathcal{B}(\Upsilon(1 S) \rightarrow \bar{D}+X)=(2.9 \pm 0.3) \times 10^{-5}$ and $\mathcal{B}(\Upsilon(2 S) \rightarrow$

All of the above-mentioned candidates for exotic states have been the subject of numerous theoretical and ex-

\footnotetext{
1) The rates for deuteron and antideuteron production in $\Upsilon(n S)$ decays are almost certainly equal. However, in $e^{+} e^{-}$experiments, background deuterons are copiously produced by particle interactions in the beam pipe and other material in the inner part of the detector, and these make experimental measurements of their true rate quite difficult. Therefore the rate is quoted for antideuterons, which, because they do not suffer from these backgrounds, are easier to measure.
} 
$\bar{D}+X)=(3.4 \pm 0.6) \times 10^{-5}[13]$. If the six-quark $H$ dibaryon is produced at a rate that is similar to that for six-quark antideuterons, there should be many thousands of them in the 102 million $\Upsilon(1 S)$ and 158 million $\Upsilon(2 S)$ event samples collected by Belle.

For $H$ masses below $2 m_{\Lambda}$, Belle searched for $H \rightarrow$ $\Lambda p \pi^{-}\left(\& \bar{H} \rightarrow \bar{\Lambda} \bar{p} \pi^{+}\right)$signals in the inclusive $\Lambda p \pi^{-}$invariant mass distribution. For masses above $2 m_{\Lambda}$, the $H \rightarrow \Lambda \Lambda(\& \bar{H} \rightarrow \bar{\Lambda} \bar{\Lambda})$ mode was used. Figure 2 shows the measured $\Lambda p \pi^{-}$(a) \& $\bar{\Lambda} \bar{p} \pi^{+}$(b) invariant mass spectra for masses below $2 m_{\Lambda}$ and the $\Lambda \Lambda$ (c) and $\bar{\Lambda} \bar{\Lambda}$ (d) mass spectra for masses above $2 m_{\Lambda}$. Here results from the $\Upsilon(1 S)$ and $\Upsilon(2 S)$ data samples are combined. No signal is observed. The solid red curves show results of a background-only fit to the data; the dashed curve shows the MC expectations for an $H$-dibaryon produced at $1 / 20$ th of the antideuteron rate. Upper limits on the inclusive branching ratios that are at least a factor of twenty below that for antideuterons are set over the entire $\left|M_{H}-2 m_{\Lambda}\right|<30 \mathrm{MeV}$ mass interval.

Neither pentaquarks nor the $H$-dibaryon are seen in spite of the stong theoretical motivation for their exis-

tence. The absence of pentaquarks led Wilczek to remark "The story of the pentaquark shows how poorly we understand QCD" [14]. The absence of any evidence for the $H$-dibaryon (among other things) led Jaffe to observe that "The absence of exotics is one of the most obvious features of QCD" [15].

\section{What we do see}

Although forty years of experimental searches has failed to come up with compelling evidence for specifically QCD-motivated exotic hadrons, strong evidence for mesons that do not fit into the simple $q \bar{q}$ scheme of the original quark model has been steadily accumulating during the past decade. These include a candidate for a bound state of a proton and antiproton from the BESII experiment [16], so-called "baryonium", an idea that has been around for a long, long time [17], and the $X Y Z$ mesons, charmoniumlike and bottomoniumlike states that do not fit into any of the remaining unfilled states in the $c \bar{c}$ - and $b \bar{b}$-meson level schemes [18].

(a)

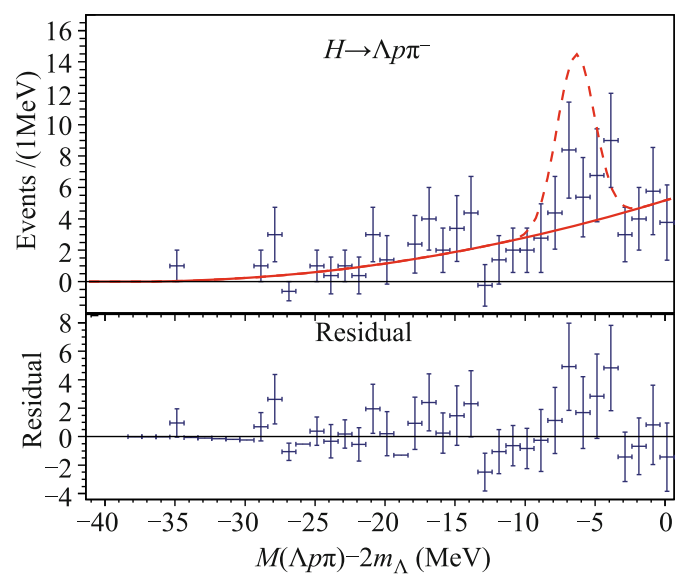

(c)

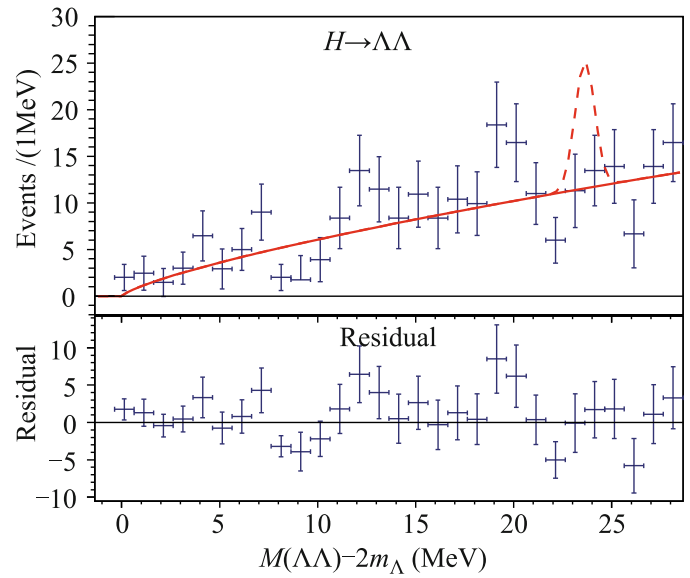

(b)

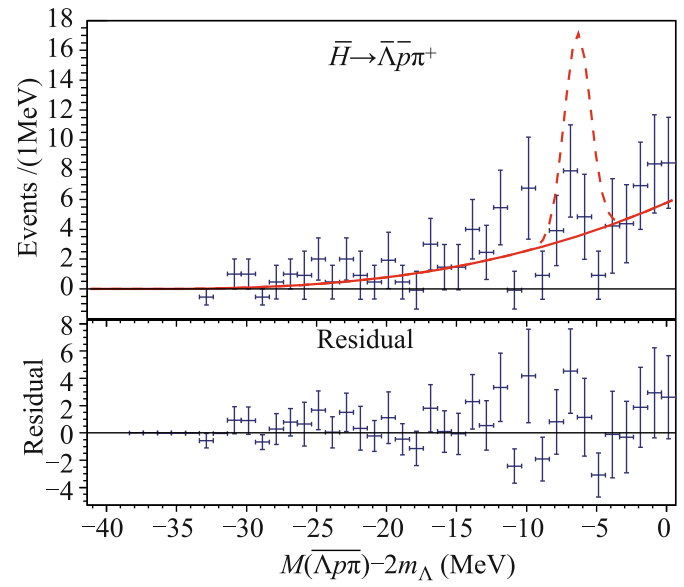

(d)

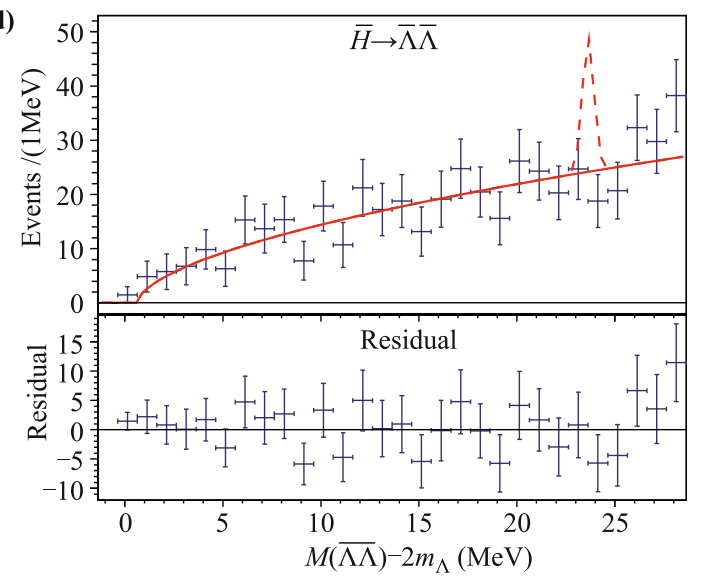

Fig. 2 (a)-(d) The $\Lambda p \pi^{-}, \bar{\Lambda} \bar{p} \pi^{+}, \Lambda \Lambda$ and $\bar{\Lambda} \bar{\Lambda}$ invariant mass distributions. The solid curves show background-only fit results and the lower panels show the fit residuals. The dashed curves are expected signals for an $H$ production rate that is $1 / 20$ th that for $\bar{D}$ 's. Reproduced from Ref. [12]. 


\subsection{Baryonium in radiative $J / \psi \rightarrow \gamma p \bar{p}$ decays?}

In 2003, the BESII experiment reported the observation of a dramatic near-threshold mass enhancement in the $p \bar{p}$ invariant mass spectrum in radiative $J / \psi \rightarrow \gamma p \bar{p}$ decays, shown in the top panel of Fig. 3(a) [16]. The lower panel in Fig. 3(a) shows the $M(p \bar{p})$ spectrum with the effects of phase-space divided out (assuming an $S$-wave $p \bar{p}$ system). It seems apparent from the phase-space-corrected plot that the dynamical source for this enhancement, whatever it may be, is at or below the $2 m_{p}$ mass threshold. A fit with a Breit-Wigner (BW) line shape modified by a kinematic threshold factor yielded a peak mass of $1859_{-27}^{+6} \mathrm{MeV}$, about $18 \mathrm{MeV}$ below $2 m_{p}$, and an upper limit of $\Gamma<30 \mathrm{MeV}$ on the width ${ }^{2)}$. It was subsequently pointed out that the BW form used by BESII should be modified to include the effect of final-state-interactions on the shape of the $p \bar{p}$ mass spectrum $[19,20]$. When this was done, it was found that the effects of FSI are not sufficient to explain the observed structure and the peak mass of the BW term shifted downward, from 1859 $\mathrm{MeV}$ to $1831 \pm 7 \mathrm{MeV}$ while the range of allowed widths increased to $\Gamma<153 \mathrm{MeV}$.

Soon after the BESII publication appeared, Yan and Ding proposed a Skyrme-like model for protonantiproton interactions in which the BESII $p \bar{p}$ massthreshold enhancement is an $S$-wave $p \bar{p}$ bound state with binding energy around $20 \mathrm{MeV}$ [21]. Since the $p$ and the $\bar{p}$ in such a system would annihilate whenever they came within close proximity of each other, such a state would have a finite width, creating a situation illustrated by the cartoon in panel (b) of Fig. 3: for masses above the $2 m_{p}$ threshold, the state would decay essentially $100 \%$ of the time by "falling apart" into a $p$ and $\bar{p}$; for masses below $2 m_{p}$, the decay would proceed via $p \bar{p}$ annihilation into mesons. Since a preferred channel for low-energy $S$ wave $p \bar{p}$ annihlation is $\pi^{+} \pi^{-} \eta^{\prime}$, Yan and Ding advocated a search for $\pi^{+} \pi^{-} \eta^{\prime}$ decays of this same state in radiative $J / \psi \rightarrow \gamma \pi^{+} \pi^{-} \eta^{\prime}$ decays. A subsequent BESII study of $J / \psi \rightarrow \gamma \pi^{+} \pi^{-} \eta^{\prime}$ decays found a distinct peak at $1834 \pm 7$ $\mathrm{MeV}$ and width $68 \pm 21 \mathrm{MeV}$ as shown in panel (c) of Fig. 3 [22], in good agreement with the mass and width results from the FSI-corrected fit to the $p \bar{p}$ mass spectrum.

The $p \bar{p}$ mass-threshold enhancement was confirmed at the same mass with much higher statistics by BESIII [23]. The signal, shown in panel (d) of Fig. 3, has a significance that is $>30 \sigma^{3)}$. The large BESIII event sam- ple permitted the application of a partial wave analysis (PWA) that established the $J^{P C}=0^{-+}$quantum number assignment, in agreement with baryonium expectations. The $\pi^{+} \pi^{-} \eta^{\prime}$ peak in $J / \psi \rightarrow \pi^{+} \pi^{-} \eta^{\prime}$ decays was also confirmed and the production-angle distribution was found to be consistent with a $J^{P C}=0^{-+}$assignment. However, the situation still remains unclear. The BESIII measurements find a much larger width for the $\pi^{+} \pi^{-} \eta^{\prime}$ peak than that found for the $p \bar{p}$ peak in the BESIII partial wave analysis: $\Gamma_{\pi^{+} \pi^{-} \eta^{\prime}}=190 \pm 38 \mathrm{MeV}$ versus $\Gamma_{p \bar{p}}<76 \mathrm{MeV}$. Another puzzling feature is the lack of any evidence for the $p \bar{p}$ threshold enhancement in any other channels, such as $J / \psi \rightarrow \omega p \bar{p}[25], \Upsilon(1 S) \rightarrow \gamma p \bar{p}$ [26] or in $B$ decays [27]. BESIII is actively looking at various other radiative $J / \psi$ decay channels for evidence for or against other signs of resonance behaviour near 1835 $\mathrm{MeV}[28]$.

\subsection{The $X Y Z$ mesons}

The $X Y Z$ mesons are a class of (somewhat haphazardly named) hadrons that are seen to decay to final states that contain a heavy quark and a heavy antiquark, i.e., $Q$ and $\bar{Q}$, where $Q$ is either a $c$ or $b$ quark, but cannot be easily accommodated in an unassigned $Q \bar{Q}$ level. Since the $c$ and $b$ quarks are heavy, their production from the vacuum in the fragmentation process is heavily suppressed and, thus, any heavy quarks seen among the decay products of a hadron must have been present among its original constituents. In addition, the heavy quarks in conventional $Q \bar{Q}$ "quarkonium" mesons are slow and can be described reasonably well by non-relativistic quantum mechanics. Indeed it was the success of non-relativistic charmonium potential-model descriptions of the $\psi$ and $\chi_{c}$ states in the mid-1970's that led to the general acceptance of the reality of quarks and the validity of the quark model. Quarkonium models specify the allowed states of a $Q \bar{Q}$ system; if a meson decays to a final state containing a $Q$ and a $\bar{Q}$ but does not match the expected properties of any of the unfilled levels in the associated $Q \bar{Q}$ spectrum, it is necessarily exotic.

\subsubsection{Charmoniumlike mesons}

Charmoniumlike $X Y Z$ mesons were first observed starting in 2003 and continue to be found at a rate of about one or more new ones every year. There is a huge theoretical and experimental literature on this subject that

\footnotetext{
2) This report only lists quadrature sums of statistical and systematic errors. Refer to the cited papers for details.

3) With apologies to Tommaso Dorigo: http://www.science20.com/a_quantum_diaries_survivor/a_useful_approximation_for_the_tail_of _a_gaussian-141353
} 
(a)

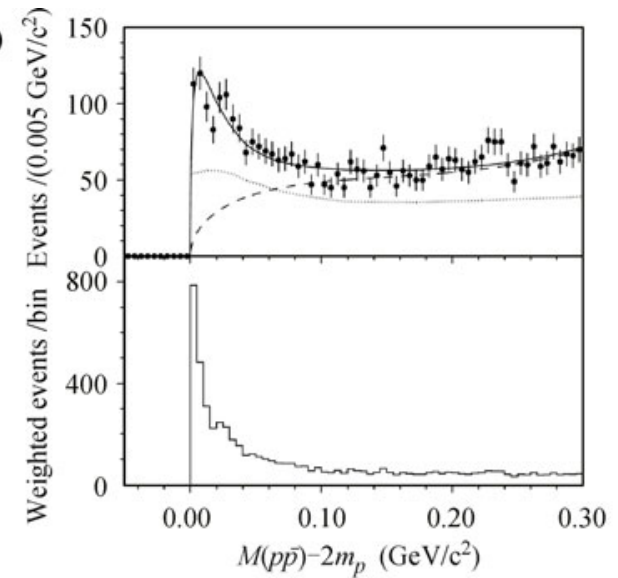

(c)

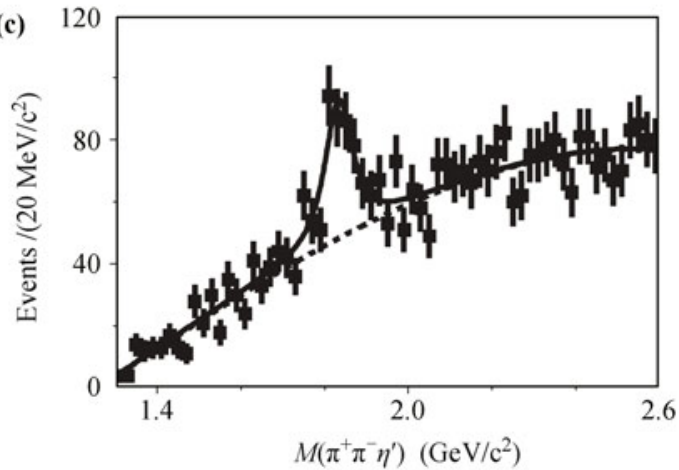

(b)

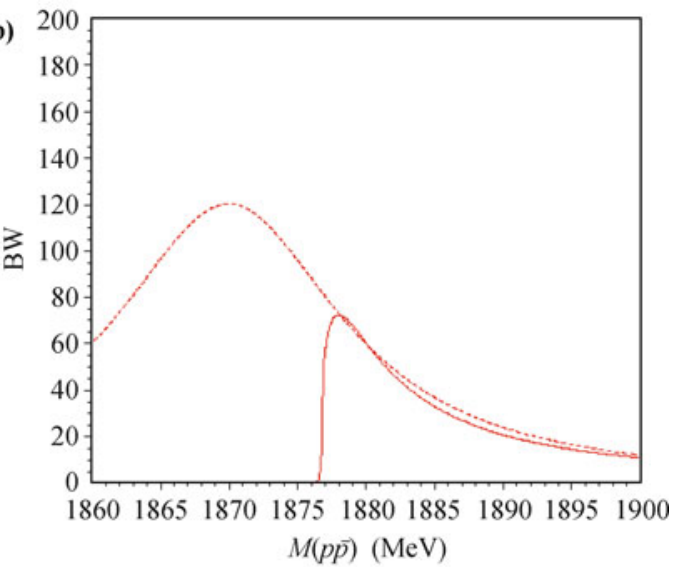

(d)

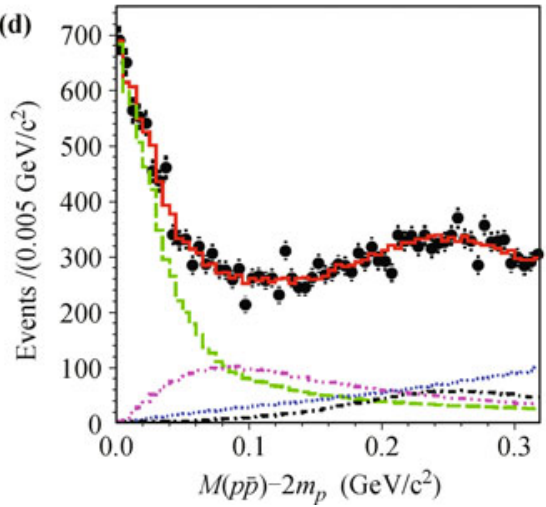

Fig. 3 (a) The upper panel shows the $M(p \bar{p})$ distribution for $J / \psi \rightarrow \gamma p \bar{p}$ decays from BESII [16]. The lower panel shows the same distribution with the kinematic threshold suppression factor removed. (b) A cartoon showing a BW line shape and a threshold-attenuated BW line-shape for a hypothesized baryonium state. The solid curve shows the expected $p \bar{p}$ line shape. Below threshold, where the dashed curve dominates, the state decays via $p \bar{p}$ annihilation into mesons. (c) The $M\left(\pi^{+} \pi^{-} \eta^{\prime}\right)$ mass distribution for $J / \psi \rightarrow \gamma \pi^{+} \pi^{-} \eta^{\prime}$ decays from Ref. [22]. (d) The $M(p \bar{p})$ distribution for $J / \psi \rightarrow \gamma p \bar{p}$ from BESIII [23] with PWA results shown as histograms.

will not be repeated here $[29,30]$. Instead I restrict myself to a few remark on subsets of the measurements and outstanding issues.

Figure 4 shows the current state of the charmonium and charmoniumlike meson spectrum below $4500 \mathrm{MeV}$. Here the yellow boxes indicate established charmonium states. All of the (narrow) states below the $2 m_{D}$ opencharm threshold have been established and found to have properties that are well described by the charmonium model. In addition, all of the $J^{P C}=1^{--}$states above the $2 m_{D}$ open-charm threshold have also been identified. The gray boxes show the remaining predicted, but still unassigned, charmonium states. The red boxes show electrically neutral $X$ and $Y$ mesons and the purple boxes show the charged $Z$ mesons, aligned according to my best guess at their $J^{P C}$ quantum numbers. In the following, I briefly comment on each of the $X Y Z$ entries in (roughly) clockwise order, starting at the left with the $X(3940)$ and $X(4160)$.

$\boldsymbol{X}(\mathbf{3 9 4 0 )}$ and $\boldsymbol{X}(\mathbf{4 1 6 0 )}$ The $X(3940)$ was first seen by Belle [31] as a peak in the distribution of masses $\left(M_{X}\right)$ recoiling against a $J / \psi$ in inclusive $e^{+} e^{-} \rightarrow J / \psi X$ annihilations at $\sqrt{s} \simeq 10.6 \mathrm{GeV}$ shown in panel (a) of Fig. 5. In this figure, there are four distinct peaks: the lower three are due to the exclusive processes $e^{+} e^{-} \rightarrow J / \psi \eta_{c}$, $e^{+} e^{-} \rightarrow J / \psi \chi_{c 0}$ and $e^{+} e^{-} \rightarrow J / \psi \eta_{c}^{\prime}$. The fourth peak near $3940 \mathrm{MeV}$ cannot be associated with any known or expected charmonium state and has been named the $X(3940)$. The curve shows results of a fit that includes the three known charmonium states plus a fourth state; the fit returned a mass $M=3943 \pm 6 \mathrm{MeV}$ and an upper limit on the total width of $\Gamma \leqslant 52 \mathrm{MeV}$.

Belle also did a study of exclusive $e^{+} e^{-} \rightarrow$ $J / \psi D^{(*)} \bar{D}^{(*)}$ decays in the same energy region. Here, to compensate for the low detetction efficiency for $D$ and $D^{*}$ mesons, a partial reconstruction technique was used that required the reconstruction of the $J / \psi$ and only one $D$ or $D^{*}$ meson ${ }^{4}$, and determined the presence of the $\bar{D}$ or $\bar{D}^{*}$ from energy momentum conservation [32]. With this technique, the $X(3940)$ was seen in the $D \bar{D}^{*}$

\footnotetext{
4) In the remainder of this report, the inclusion of charge conjugate states is always implied.
} 


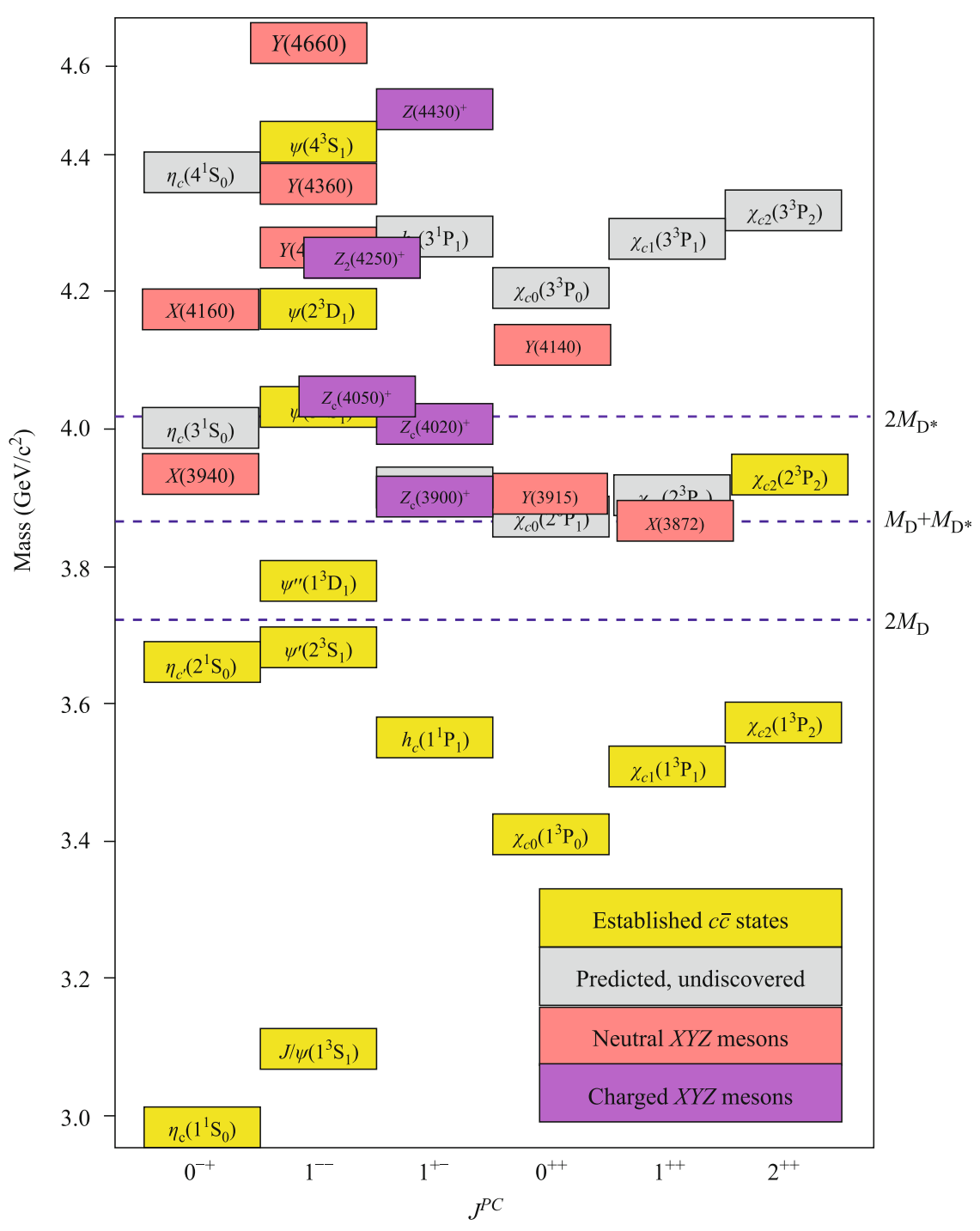

Fig. 4 The spectrum of charmonium and charmoniumlike mesons

invariant mass distribution in $e^{+} e^{-} \rightarrow J / \psi D \bar{D}^{*}$ annihilations [32], as can be seen in the lower panel of Fig. 5(b) and the upper panel in Fig. 5(c). Here the fits shown in the plots return a mass and width of $M=3942 \pm 9 \mathrm{MeV}$ and $\Gamma=37_{-17}^{+27} \mathrm{MeV}$. The $e^{+} e^{-} \rightarrow J / \psi D^{*} \bar{D}^{*}$ study uncovered another, higher mass state decaying to $D^{*} \bar{D}^{*}$ as can be seen in the lower panel of Fig. 5(c). The fitted mass and width of this state, which is called the $X(4160)$, is $M=4156 \pm 27 \mathrm{MeV}$ and $\Gamma=139_{-65}^{+113} \mathrm{MeV}$ [32].

Neither the $X(3940)$ nor the $X(4160)$ show up in the $D \bar{D}$ invariant mass distribution for exclusive $e^{+} e^{-} \rightarrow$ $J / \psi D \bar{D}$ at the same energies. Instead, the $M(D \bar{D})$ spectrum exhibits a broad excess of events over an equally broad background as shown in the upper panel of Fig. 5(b). A fit to a resonant shape, shown as a curve in the figure, returns a signal of marginal significance $(3.8 \sigma)$ with a peak mass of $M=3780 \pm 48 \mathrm{MeV}$ and a width $\Gamma=347_{-143}^{+316} \mathrm{MeV}$. Since the fitted values are unstable under variations of the background shape parameterization and the bin size, Belle makes no claims about this distribution other than that it is inconsistent with phase space or pure background. On the other hand, Chao [33] suggests that this may be the $\chi_{c 0}^{\prime}$, which is discussed below in conjunction with the $X(3915)$.

The absence of signals for any of the known spin nonzero charmonium states in the inclusive spectrum of Fig. 5 (a) provides circumstantial evidence for $J=0$ assignments for the $X(3940)$ and $X(4160)$. The $X(3940) \rightarrow$ $D^{*} \bar{D}$ decay mode then ensures that its $J^{P C}$ values are $0^{-+}$. The absence of any signal for $X(4160) \rightarrow D \bar{D}$ decay supports a $0^{-+}$assignment for this state as well. In both cases, the measured masses are far below expectations for the only available $0^{-+}$charmonium levels: the $\eta_{c}(3 S)$ and $\eta_{c}(4 S)$. Since there are no strong reasons to doubt the generally accepted identifications of the $\psi(4040)$ peak seen in the inclusive cross section for 

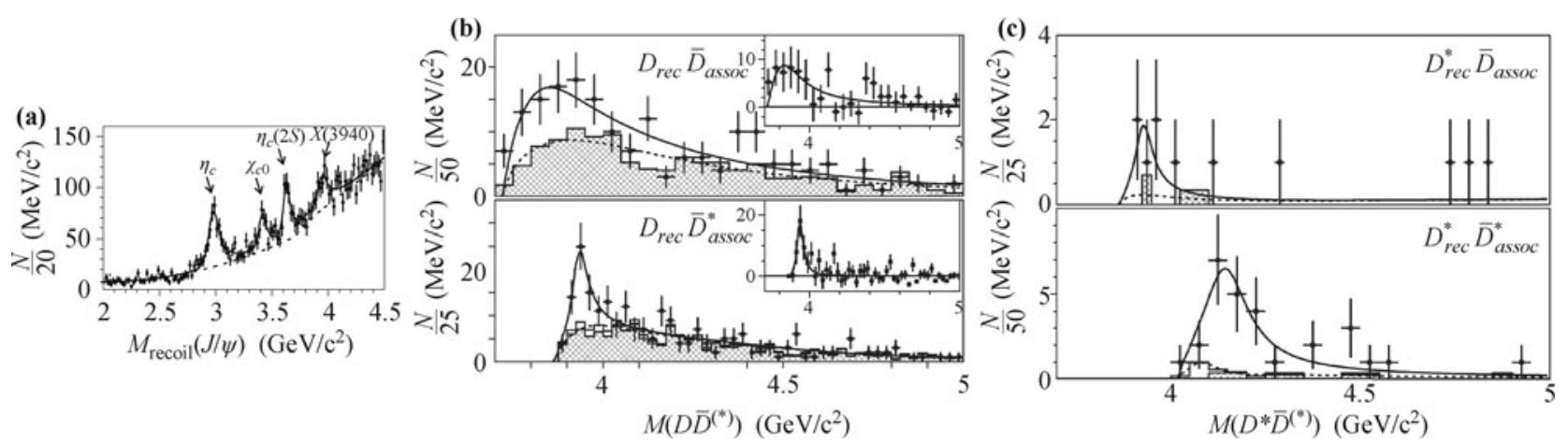

Fig. 5 (a) The distribution of masses recoiling from a $J / \psi$ in inclusive $e^{+} e^{-} \rightarrow J / \psi X$ annihilations near $\sqrt{s} \simeq 10.6 \mathrm{GeV}$. The curve is the result of a fit described in the text. (b) The $D \bar{D}$ (upper) and $D \bar{D}^{*}$ (lower) invariant mass distributions from $e^{+} e^{-} \rightarrow J / \psi D \bar{D}^{(*)}$ annihilations near $\sqrt{s} \simeq 10.6 \mathrm{GeV}$. Here the $J / \psi$ and a $D$ meson are reconstructed, and the presence of the $\bar{D}$ or $\bar{D}^{*}$ is inferred from kinematics. (c) The $D^{*} \bar{D}$ (upper) and $D^{*} \bar{D}^{*}$ (lower) invariant mass distributions from $e^{+} e^{-} \rightarrow J / \psi D^{*} \bar{D}^{(*)}$ annihilations near $\sqrt{s} \simeq 10.6 \mathrm{GeV}$. Here the $J / \psi$ and a $D^{*}$ are reconstructed and the presence of the $\bar{D}$ or $\bar{D}^{*}$ is inferred from kinematics. The hatched histograms in the center and right panels show backgrounds estimated from the $J / \psi$ and $D^{(*)}$ mass sidebands; the insets in the center panels show background-subtracted fits.

$e^{+} e^{-} \rightarrow$ hadrons as the $\psi(3 S)$ and the $\psi(4415)$ peak as the $\psi(4 S)[34,35]$, these assignments would imply hyperfine $n^{3} S-n^{1} S$ mass splittings that increase from the measured value of $47.2 \pm 1.2 \mathrm{MeV}$ for $n=2$ [13], to $\sim 100$ $\mathrm{MeV}$ for $n=3$ and $\sim 350 \mathrm{MeV}$ for $n=4$ [33]. This pat- tern conflicts with expectations from potential models, where hyperfine splittings are proportional to the square of the radial wavefunction at $r=0$ and decrease with increasing $n$ [36].

$\boldsymbol{Y}(4260), \quad \boldsymbol{Y}(4360)$ and $\quad \boldsymbol{Y}(4660): \quad$ BaBar
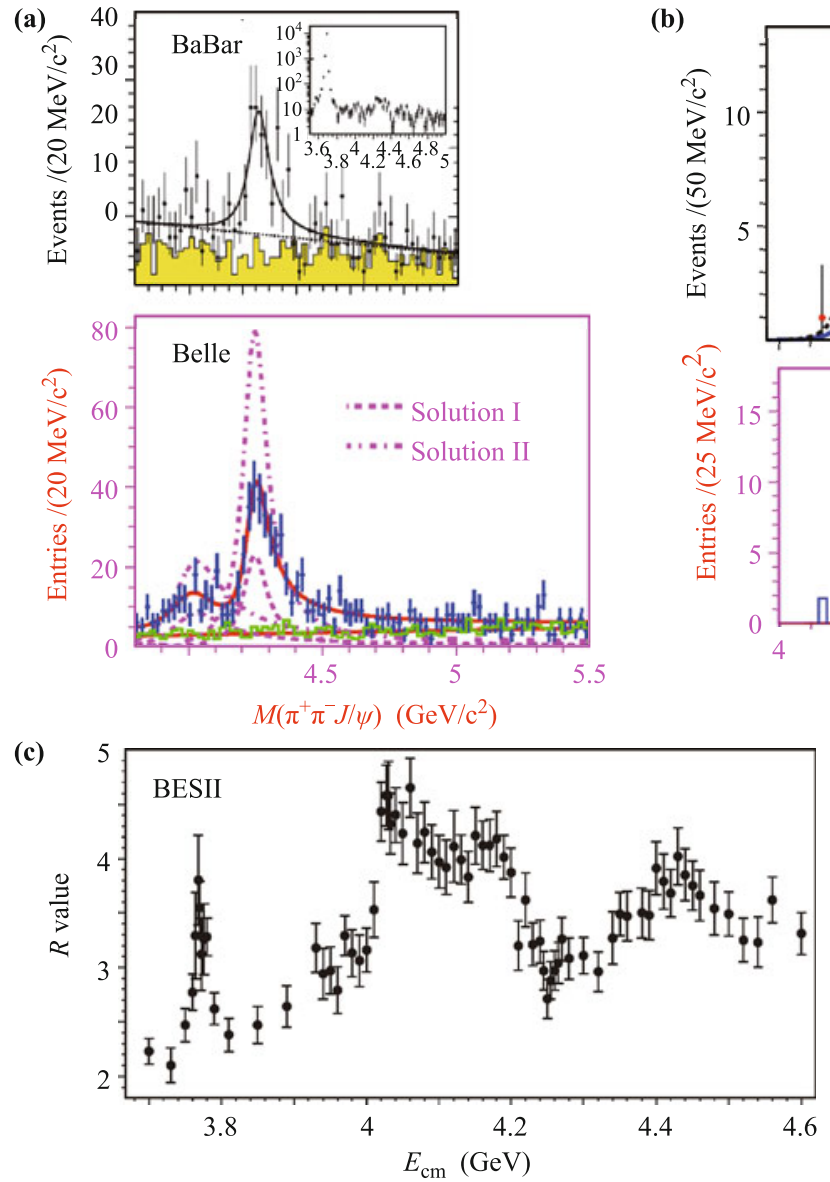

(b)

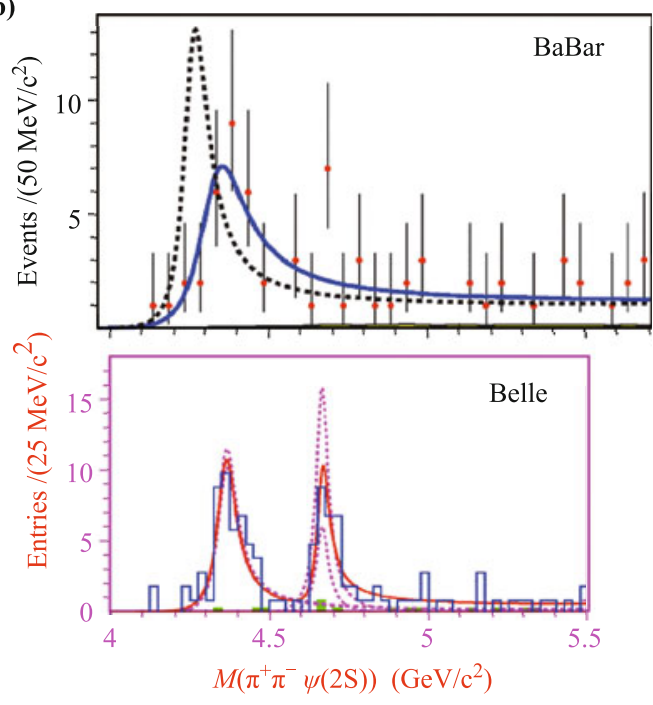

Fig. 6 (a) The $M\left(\pi^{+} \pi^{-} J / \psi\right)$ from $e^{+} e^{-} \rightarrow \gamma_{\text {isr }} \pi^{+} \pi^{-} J / \psi$ events from BaBar (upper) and Belle (lower). (b) The $M\left(\pi^{+} \pi^{-} \psi^{\prime}\right)$ from $e^{+} e^{-} \rightarrow \gamma_{\mathrm{isr}} \pi^{+} \pi^{-} \psi^{\prime}$ events from BaBar (upper) and Belle (lower). (c) The total inclusive Born cross section for $e^{+} e^{-} \rightarrow$ hadrons in units of $\sigma_{\mathrm{QED}}\left(e^{+} e^{-} \rightarrow \mu^{+} \mu^{-}\right)$from BESII. 
discovered the $Y(4260)$ as a peak near $4260 \mathrm{MeV}$ in the $M\left(\pi^{+} \pi^{-} J / \psi\right)$ distribution from initial-state-radiation $e^{+} e^{-} \rightarrow \gamma_{\text {isr }} \pi^{+} \pi^{-} J / \psi$ events [37] shown in the upper panel of Fig. $6(\mathrm{a})$, and the $Y(4360)$ in the $\pi^{+} \pi^{-} \psi^{\prime}$ system produced via $e^{+} e^{-} \rightarrow \gamma_{\mathrm{isr}} \pi^{+} \pi^{-} \psi^{\prime}$ [38], shown in the upper panel of Fig. 6(b). These states were confirmed by Belle, as shown in the lower panels of the same figures $[39,40]$. Belle found another peak, the $Y(4660)$, at higher mass in the $M\left(\pi^{+} \pi^{-} \psi^{\prime}\right)$ distribution. There is no sign of the $Y(4260)$ in the $M\left(\pi^{+} \pi^{-} \psi^{\prime}\right)$ distributions: the expected shape for $Y(4260) \rightarrow \pi^{+} \pi^{-} \psi^{\prime}$ is shown as a dashed curve in the upper panel of Fig. 6(b). Neither is there any sign of the $Y(4360)$ or $Y(4660)$ in the $M\left(\pi^{+} \pi^{-} J / \psi\right)$ distributions of Fig. 6(a).

These states have to be considered exotic because their production mechanism ensures that $J^{P C}=1^{--}$and all of the $1^{--} c \bar{c}$ states near their masses have already been assigned. Moreover, there is no evidence for them in any exclusive [41-44] or the inclusive [34, 35] charmed-meson production cross section, where there is a pronounced dip at $\sqrt{s} \simeq 4.26 \mathrm{GeV}$ and no striking feature near $4.36 \mathrm{GeV}$, as can be seen in BESII cross section data shown in Fig. $6(\mathrm{c})[34,35]$. This implies large partial decay widths to $\pi^{+} \pi^{-} J / \psi\left(\psi^{\prime}\right)$; for example: a specific analysis for the $Y(4260)$ finds $\Gamma\left(Y(4260) \rightarrow \pi^{+} \pi^{-} J / \psi\right)>1 \mathrm{MeV}$ [45], which is huge by charmonium standards. Some authors have proposed that the $Y(4260)$ is a $c \bar{c}$-gluon hybrid state $[46,47]$ while others have suggested that it is a molecule-like $D \bar{D}_{1}(2420)$ bound-state [48]. The $Y(4260)$ is discussed in more detail below.

The electrically charged $Z_{c}(3900), Z_{c}(4020)$, $Z_{1}$ (4050), $Z_{2}(4250)$ and $Z(4430)$ states: Since the $Z_{c}(3900)[49,50], Z_{c}(4020)[51,52], Z_{1}(4050)$ [53], $Z_{2}(4250)$ [53] and $Z(4430)$ [54] are electrically charged and decay to hidden charm final states, their minimal quark structure is a $c \bar{c} u \bar{d}$ four-quark combination and, therefore, if they are mesons, they must be exotic.

The $Z$ (4430): The first electrically charged, charmoniumlike state to be reported was the $Z$ (4430), which was found by Belle in 2008 in a study of $B \rightarrow K \pi^{+} \psi^{\prime}$ decays [54]. An $M^{2}\left(\pi^{+} \psi^{\prime}\right)$ vs. $M^{2}\left(K \pi^{+}\right)$Dalitz plot for these decays is shown in Fig. 7(a). There, the most prominent feature is a strong vertical band near $M^{2}\left(K \pi^{+}\right) \simeq 0.8$ $\mathrm{GeV}^{2}$ corresonding to $B \rightarrow K^{*}(890) \psi^{\prime}$ decays. A second vertical band near $M^{2}\left(K \pi^{+}\right) \simeq 2 \mathrm{GeV}^{2}$ corresponds to $B \rightarrow K_{2}^{*}(1430) \psi^{\prime}$. To reduce the influence of these structures, Belle studied events with $M\left(K \pi^{+}\right)$more than 100 $\mathrm{MeV}$ away from the $K^{*}(890)$ and $K_{2}^{*}(1430)$ peak mass values (the $K^{*}$ veto). The $\pi^{+} \psi^{\prime}$ invariant mass distri- bution in $B \rightarrow K \pi^{+} \psi^{\prime}$ decay events that survive this $K^{*}$ veto, shown an open histogram in Fig. 7(b), exhibits a distinct peak near $M\left(\pi^{+} \psi^{\prime}\right) \simeq 4.43 \mathrm{GeV}$. A fit to a smooth background plus a BW signal function to describe the peak returns a mass and width of $M=4433 \pm 5$ $\mathrm{MeV}$ and $\Gamma=45_{-18}^{+35} \mathrm{MeV}$; the statistical significance of the peak is $6.5 \sigma$. However, a 2009 analysis by BaBar failed to confirm the Belle signal [55]. Figure 7(c), taken from the BaBar paper, presents a direct comparison between the Belle and BaBar results with the same $K^{*}$ veto requirements. A BW fit by BaBar that used Belle's $Z$ (4430) mass and width values found a signal with a statistical significance of only $\simeq 2 \sigma$.

One concern about the $Z(4430)$ was that neither the BaBar nor the 2008 Belle analysis considered the possibility of interference between $B \rightarrow K Z(4430)$ and $B \rightarrow K^{*} \psi^{\prime}$ amplitudes. Because of this, Belle did two subsequent analyses that explicitly accounted for the possibility of such interference $[56,57]$. The decay chain $B \rightarrow K \pi^{+} \psi^{\prime} ; \psi^{\prime} \rightarrow \ell^{+} \ell^{-}$can be completely described by four variables ${ }^{5)}$, which can be taken as $M\left(\pi^{+} \psi^{\prime}\right)$, $M\left(K \pi^{+}\right)$, the $\psi^{\prime}$ helicity angle $\theta$ and the angle between the $K \pi^{+}$and $\ell^{+} \ell^{-}$planes in the $B$ rest frame $\phi$. Belle's first amplitude analysis integrated over $\theta$ and $\phi$ and only considered the $K \pi^{+}$and $\pi^{+} \psi^{\prime}$ masses [56]; the second analysis used all four variables [57]. Both amplitude analyses found strong signals (6.4 $\sigma$ in both cases) for a resonance in the $\pi^{+} \psi^{\prime}$ channel, with a somewhat higher peak mass and much broader width than that reported in the 2008 Belle paper; the four-dimensional analysis, based on $2010 \pm 50 B \rightarrow K \pi^{+} \psi^{\prime}$ signal events, reported $M=4485 \pm_{-25}^{+36} \mathrm{MeV}$ and $\Gamma=200_{-58}^{+49} \mathrm{MeV}$. These amplitude analyses show strong interference between the $Z \rightarrow \pi^{+} \psi^{\prime}$ and $K^{*} \rightarrow K \pi^{+}$channels, as is evident in Fig. 8(a) where the $M^{2}\left(\pi^{+} \psi^{\prime}\right)$ distribution for the data surviving the $K^{*}$ veto (points with errors) are shown with projections of the four-dimensional fit results superimposed $^{6)}$. The fit that includes the $Z^{+} \rightarrow \pi^{+} \psi^{\prime}$ resonance is shown as a solid histogram and the best fit with no $Z^{+}$resonance is shown as a dashed curve. For masses below the $Z^{+}$resonance peak, the intereference is constructive and there is a clear positive enhancement; for masses above the peak the interference is destructive and produces a depletion of events. The original Belle result, which was based on a one-dimensional fit to the $M\left(\pi^{+} \psi^{\prime}\right)$ distribution, only fit the low-mass positive lobe and this resulted in a lower mass and a narrower width. The four-dimensional analysis strongly favored the $J^{P}=1^{+}$quantum number assignment for

\footnotetext{
5) This neglects the finite width of the $\psi^{\prime}$ resonance.

6) For the four-dimensional analysis, only the $\psi^{\prime} \rightarrow \ell^{+} \ell^{-}$decay channel was used.
} 

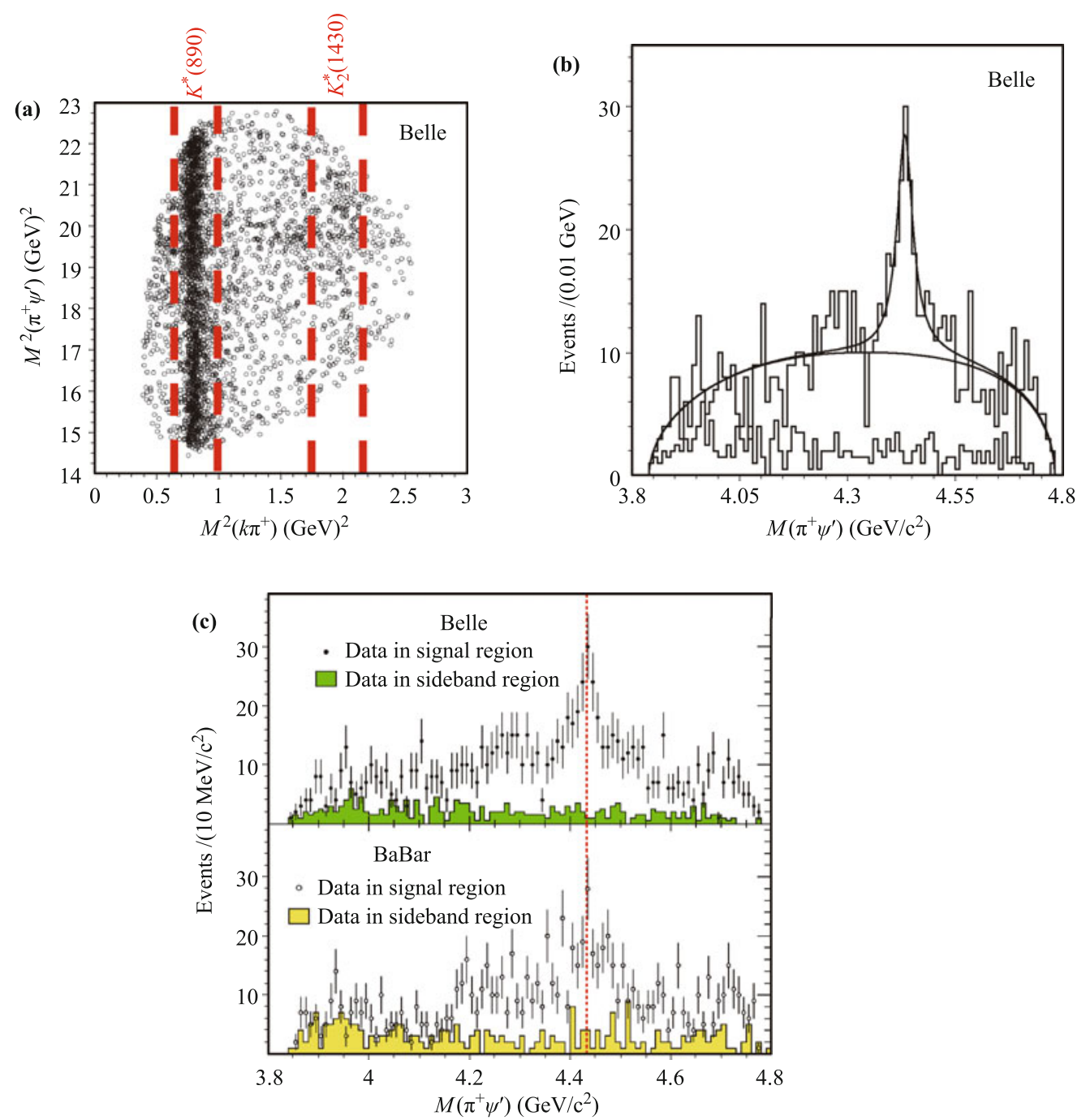

Fig. 7 (a) An $M^{2}\left(\pi^{+} \psi^{\prime}\right)$ (vertical) vs. $M^{2}\left(K \pi^{+}\right)$(horizontal) Dalitz plot for $B \rightarrow K \pi^{+} \psi^{\prime}$ events from Belle. The vertical dashed red lines indicate the boundaries of $K^{*}$ veto requirement described in the text. (b) The $\pi^{+} \psi^{\prime}$ invariant mass distribution from $B \rightarrow K \pi^{+} \psi^{\prime}$ decays from Belle [54] for events with the $K^{*}$ veto requirement applied is shown as the open histogram. The shaded histogram is non- $\psi^{\prime}$ background, estimated from the $\psi^{\prime}$ mass sidebands. The curves represent results of a fit described in the text. (c) A comparison of the Belle data (upper) and BaBar data (lower) [55] with the the $K^{*}$ and $K_{2}^{*}$ vetoed.

the $Z(4430)$.

In 2014, the LHCb experiment repeated the Belle fourdimensional analysis with a sample of more than $25 \mathrm{~K}$ $\bar{B}^{0} \rightarrow K^{-} \pi^{+} \psi^{\prime}$ decays [58] and confirmed the Belle signal with a significance greater than $14 \sigma$. A comparison of their $M\left(\pi^{+} \psi^{\prime}\right)$ data distribution with projections of their fit results superimposed is shown in Fig. 8(b), where strong interference effects, similar in character to those reported by Belle, are apparent. The LHCb results for the mass, width and $J^{P}$ values, $M=4475_{-26}^{+17} \mathrm{MeV}$, $\Gamma=172_{-36}^{+39} \mathrm{MeV}$ and $J^{P}=1^{+}$, agree well with the Belle four-dimensional fit results but with smaller errors. With their ten-fold larger event sample, the LHCb group was able to relax the assumption of a BW form for the $Z^{+}$ amplitude and directly measure the real and imaginary parts of the amplitude as a function of mass. The results are shown as data points in Fig. 8(c). There the phase motion near the resonance peak agrees well with expectations for a BW amplitude as indicated by the nearly circular red curve superimposed on the plot. This rapid phase motion is a clear signature for a BW-like resonance behavior but, by itself, does not necessarily rule out explanations of the $Z(4430)$ structure as being due to a rescattering process $[59,60]$.

The Belle and $\mathrm{LHCb}$ product branching fractions for $Z(4430)^{+}$production in $\bar{B}^{0}$ decays are

$$
\begin{aligned}
\mathcal{B}\left(\bar{B}^{0}\right. & \left.\rightarrow K^{-} Z(4430)^{+}\right) \times \mathcal{B}\left(Z(4430)^{+} \rightarrow \pi^{+} \psi^{\prime}\right) \\
& =6.0_{-2.4}^{+3.0} \times 10^{-5} \text { Belle, and }
\end{aligned}
$$




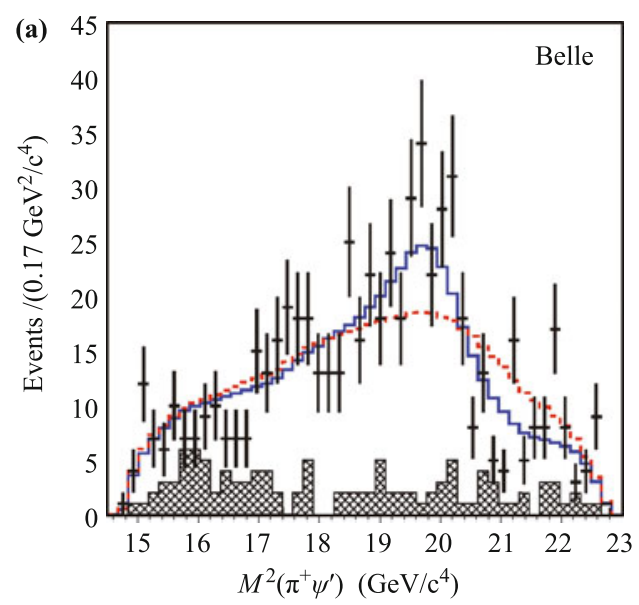

(b)
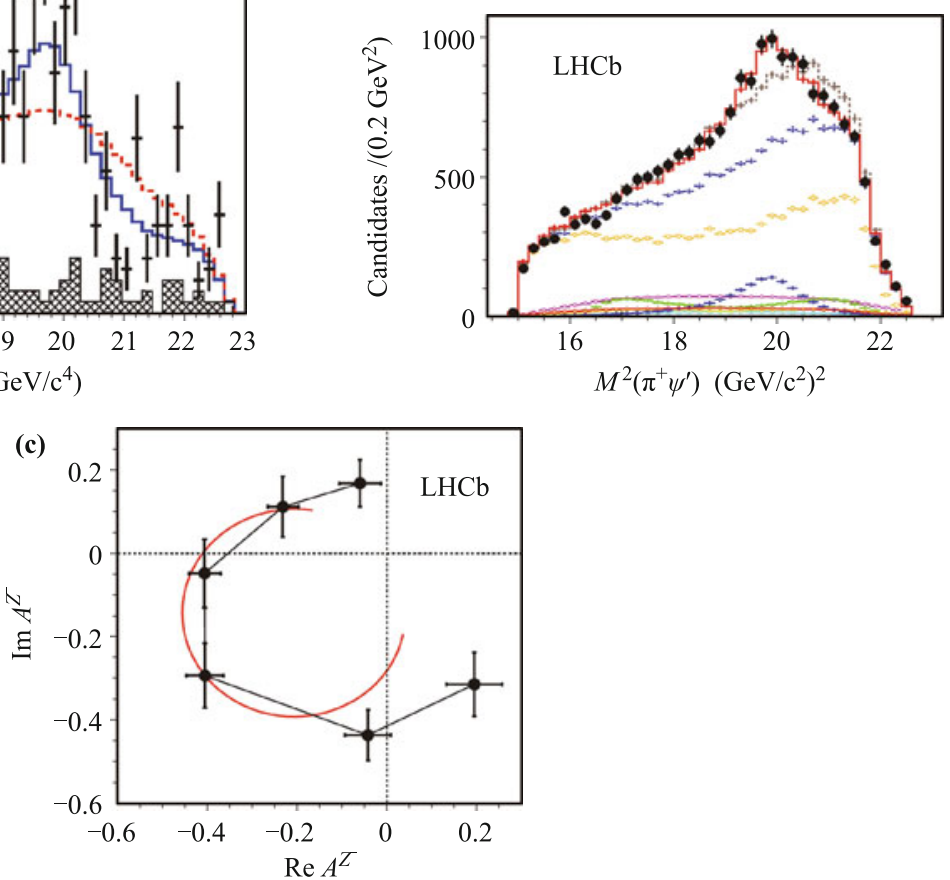

Fig. 8 (a) The data points show the Belle $M^{2}\left(\pi^{+} \psi^{\prime}\right)$ distribution with the $K^{*}$ veto applied. The solid (blue) histogram shows four-dimensional fit results with a $Z^{+} \rightarrow \pi^{+} \psi^{\prime}$ resonance included. The dashed (red) curve shows best fit results with no resonance in the $\pi^{+} \psi^{\prime}$ channel. (b) The LHCb group's $M^{2}\left(\pi^{+} \psi^{\prime}\right)$ distribution for all events (no $K^{*}$ veto), together with projections from the four-dimensional fits. The solid red histogram shows the fit that includes a $Z^{+} \rightarrow \pi^{+} \psi^{\prime}$ resonance term; the dashed brown histogram shows the fit with no resonance in the $\pi^{+} \psi^{\prime}$ channel. (c) The Real (horizontal) and Imaginary (vertical) parts of the $\left(1^{+}\right) Z^{+} \rightarrow \pi^{+} \psi^{\prime}$ amplitude for different mass bins spanning the $4430 \mathrm{MeV}$ mass region from $\mathrm{LHCb}[58]$. The red curve shows expectations for a BW resonance amplitude.

$$
\begin{aligned}
\mathcal{B}\left(\bar{B}^{0}\right. & \left.\rightarrow K^{-} Z(4430)^{+}\right) \times \mathcal{B}\left(Z(4430)^{+} \rightarrow \pi^{+} \psi^{\prime}\right) \\
& =3.4_{-2.4}^{+1.1} \times 10^{-5} \mathrm{LHCb} ;
\end{aligned}
$$

the weighted average is $4.4 \pm 1.7 \times 10^{-5}$. There is no experimental value for $\mathcal{B}\left(\bar{B}^{0} \rightarrow K^{-} Z(4430)^{+}\right)$, although we expect that it cannot be larger than $\mathcal{B}\left(B^{-} \rightarrow\right.$ $K^{-} X(3872)$ ), for which BaBar has set a $90 \%$ CL upper limit of $3.2 \times 10^{-4}$ [61]. This seems reasonable because the leading quark-line diagram for $B \rightarrow K X(3872)$ is a "factorizable" weak interaction process that is favored in $B$ meson decays, while the leading-order diagram for producing a charged charmoniumlike state is "non-factorizable", and expected to be suppressed in $B$ meson decays [63-65]. Thus, it is probably safe to expect that $\mathcal{B}\left(\bar{B}^{0} \rightarrow K Z(4430)\right)<\mathcal{B}\left(B^{-} \rightarrow K^{-} X(3872)\right)<$ $3.2 \times 10^{-4}$. In that case, the Belle-LHCb average given above give a lower limit $\mathcal{B}\left(Z(4430)^{+} \rightarrow \pi^{+} \psi^{\prime}\right)>5.3 \%$ that, when coupled with our average of the Belle and LHCb measurements of the $Z$ total width $\left(\Gamma_{Z(4430)}=\right.$
$181 \pm 31 \mathrm{MeV})$, gives a lower limit on the partial width $\Gamma\left(Z(4430)^{+} \rightarrow \pi^{+} \psi^{\prime}\right)>7.5 \mathrm{MeV}$, which is very large by charmonium standards: for example, the largest measured hadronic transition width between well established charmonium states is $\Gamma\left(\psi^{\prime} \rightarrow \pi^{+} \pi^{-} J / \psi\right)=102 \pm 3 \mathrm{keV}$ $[13]^{7)}$.

Another striking feature of the $Z(4430)$ is that its $\pi^{+} \psi^{\prime}$ partial decay width is much stronger than that for $\pi^{+} J / \psi$. In 2009, BaBar reported $\mathcal{B}\left(\bar{B}^{0} \rightarrow K^{-} Z(4430)\right) \times$ $\mathcal{B}\left(Z(4430) \rightarrow \pi^{+} J / \psi\right)<0.4 \times 10^{-5}$, an order of magnitude lower than the Belle-LHCb average value for $Z(4430) \rightarrow \pi^{+} \psi^{\prime}$. Belle recently reported the first observation of the $Z(4430) \rightarrow \pi^{+} J / \psi$ decay mode in $B \rightarrow K \pi^{+} J / \psi$ decays with a product branching fraction of $5.4_{-1.3}^{+4.1} \times 10^{-6}[62]$, which is near the BaBar upper limit and about an order of magnitude smaller than the corresponding rate for $Z(4430)^{+}$production in the $B \rightarrow K \pi^{+} \psi^{\prime}$ channel.

The $Z_{1}(4050)$ and $Z_{2}(4250)$ : The $Z_{1}(4050)$ and

7) Hadronic transitions between charmonium states are OZI suppressed. This is not the case for four-quark charmoniumlike states. 
$Z_{2}(4250)$ are resonances in the $\pi^{+} \chi_{c 1}$ channel that were found by Belle in a two-dimensional $\left(M\left(K \pi^{+}\right)\right.$ vs. $\left.M\left(\pi^{+} \chi_{c 1}\right)\right)$ amplitude analysis of $B \rightarrow K \pi^{+} \chi_{c 1}$ decays [53]. Figure $9\left(\right.$ a) shows the $M^{2}\left(\pi^{+} \chi_{c 1}\right)$ distribution for events with $1.0 \mathrm{GeV}<M\left(K \pi^{+}\right)<1.32 \mathrm{GeV}$ (upper) and $M\left(K \pi^{+}\right)>1.54 \mathrm{GeV}$ (lower) with the best fit for a model with all known $K \pi$ resonances but no resonance in the $\pi^{+} \chi_{c 1}$ channel. Figure $9(\mathrm{~b})$ shows the same data with a fit that includes one resonance in the $\pi^{+} \chi_{c 1}$ channel. Here the fit quality improves substantially; the statistical significance of the $Z \rightarrow \pi^{+} \chi_{c 1}$ term is more than $10 \sigma$. The use of different parameterizations of the $K \pi$ scalar amplitude or the inclusion of an additional $J^{P}=1^{-}$or $2^{+} K^{*}$ resonance with a mass and width left as free parameters do not reduce the significance of the $Z^{+} \rightarrow \pi^{+} \chi_{c 1}$ term to below $6 \sigma$.

It is evident in Fig. 9(b) that the inclusion of a single $Z \rightarrow \pi \chi_{c 1}$ amplitude does not reproduce the details of the data. Because of this, Belle repeated the fit with two BW amplitudes in the $Z \rightarrow \pi^{+} \chi_{c 1}$ channel. This model fits the data well, as can be seen in Fig. 9(c); the significance of the two $Z$ model relative to the single $Z$ model is $5.7 \sigma$. The masses and widths of the two $Z$ s are

$$
\begin{array}{ll}
Z_{1}(4050): & M_{1}=4051_{-43}^{+24} \mathrm{MeV}, \quad \Gamma_{1}=82_{-28}^{+51} \mathrm{MeV} \\
Z_{2}(4250): & M_{2}=4248_{-45}^{+185} \mathrm{MeV}, \quad \Gamma_{2}=177_{-72}^{+321} \mathrm{MeV} .
\end{array}
$$

A BaBar study of $B \rightarrow K \pi^{+} \chi_{c 1}$ decays did not confirm the Belle claim of resonances in the $\pi^{+} \chi_{c 1}$ channel [66]. Figure 10(a) shows BaBar's background-subtracted $M\left(\pi^{+} \chi_{c 1}\right)$ distribution for events with $1.0 \mathrm{GeV}<$ $M\left(K \pi^{+}\right)<1.32 \mathrm{GeV}$. The dashed curve shows the projection of the BaBar fit with no $Z^{+}$resonances in the $\pi^{+} \chi_{c 1}$ channel. This fit obviously overshoots the data in the $M\left(\pi^{+} \chi_{c 1}\right) \sim 3.9 \mathrm{GeV}$ region and undershoots the data near $M\left(\pi^{+} \chi_{c 1}\right) \sim 4.1 \mathrm{GeV}$. The red curve shows the result when two $Z^{+} \rightarrow \pi^{+} \chi_{c 1}$ resonance terms with the Belle mass and width values are added; this improves the agreement with the data, but not markedly. The BaBar report claims that, overall, the $M\left(\pi^{+} \chi_{c 1}\right)$ distributions in the data [including those in the regions of $M\left(K \pi^{+}\right)$that are not shown in Fig. 10(a)] are adequately described by resonances in the $K \pi$ sector only, and the inclusion of $Z^{+}$resonances does not signficantly improve the fit quality. Based on this they set $90 \% \mathrm{CL}$ product branching fraction upper limits of [66]

$$
\begin{aligned}
\mathcal{B}\left(\bar{B}^{0}\right. & \left.\rightarrow K^{-} Z_{1}(4050)^{+}\right) \times \mathcal{B}\left(Z_{1}(4050)^{+}\right. \\
& \left.\rightarrow \pi^{+} \chi_{c 1}\right)<1.8 \times 10^{-5} \text { and } \\
\mathcal{B}\left(\bar{B}^{0}\right. & \left.\rightarrow K^{-} Z_{2}(4250)^{+}\right) \times \mathcal{B}\left(Z_{2}(4250)^{+}\right. \\
& \left.\rightarrow \pi^{+} \chi_{c 1}\right)<4.0 \times 10^{-5},
\end{aligned}
$$

which are below, but not in strong contradiction with,
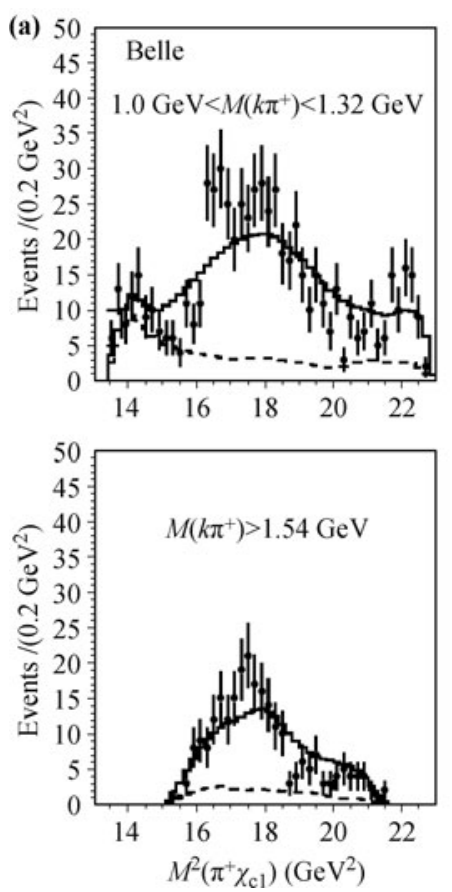
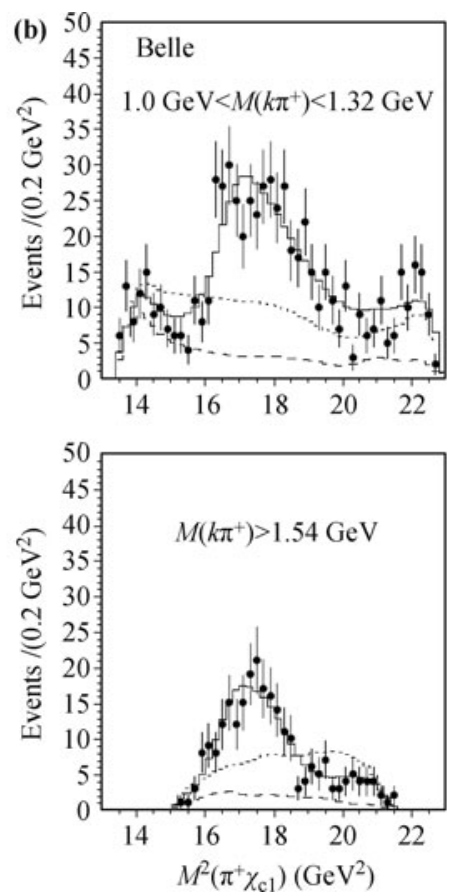
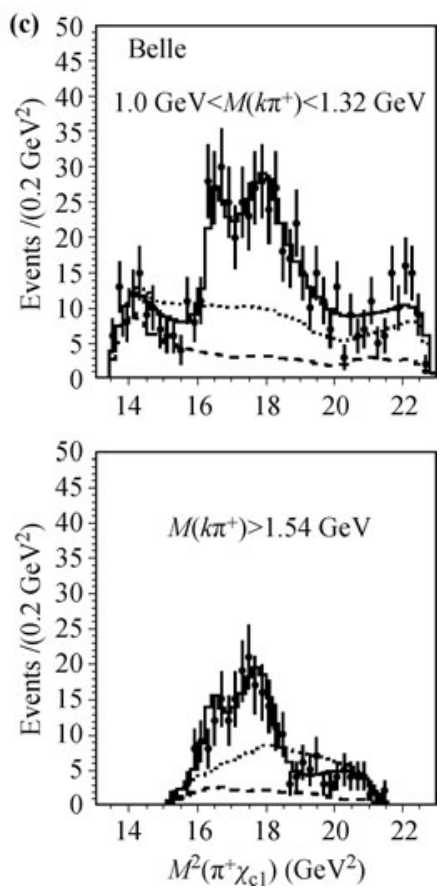

Fig. 9 (a) The $M^{2}\left(\pi^{+} \chi_{c 1}\right)$ distribution for $B \rightarrow K \pi^{+} \chi_{c 1}$ events with $1.0 \mathrm{GeV}<M\left(K \pi^{+}\right)<1.32 \mathrm{GeV}$ (upper) and $M\left(K \pi^{+}\right)>1.54 \mathrm{GeV}$ (lower) from Belle. The solid histograms are projections of the two-dimensional fit with no resonance in the $\pi^{+} \chi_{c 1}$ channel. The dashed histogram indicates the background level determined from the $\chi_{c 1}$ mass sidebands. (b) The same data with results of the fit with a single $Z^{+} \rightarrow \pi^{+} \chi_{c 1}$ resonance amplitude. Here the dotted histogram indicates the sum of all fit components other than the $Z^{+}$. (c) The same data with results of the fit that includes two $Z^{+} \rightarrow \pi^{+} \chi_{c 1}$ resonances with mass and width values given in the text. 
the Belle measurements [53]

$$
\begin{aligned}
\mathcal{B}\left(\bar{B}^{0}\right. & \left.\rightarrow K^{-} Z_{1}(4050)^{+}\right) \times \mathcal{B}\left(Z_{1}(4050)^{+}\right. \\
& \left.\rightarrow \pi^{+} \chi_{c 1}\right)=3.0_{-1.8}^{+4.0} \times 10^{-5} \text { and } \\
\mathcal{B}\left(\bar{B}^{0}\right. & \left.\rightarrow K^{-} Z_{2}(4250)^{+}\right) \times \mathcal{B}\left(Z_{2}(4250)^{+}\right. \\
& \left.\rightarrow \pi^{+} \chi_{c 1}\right)=4.0_{-1.0}^{+19.8} \times 10^{-5} .
\end{aligned}
$$

For comparison, Fig. 10(b), from Ref. [53], shows the Belle data with the same $M\left(K \pi^{+}\right)$requirement and the results of a fit with no $Z^{+}$resonances (dashed blue curve) and the fit with the two $Z^{+}$resonances described above. The Belle and BaBar data, and the results of their no $Z^{+}$fits are qualitatively very similar, while the results of the fits that include $Z^{+}$terms are quite different. This is because in Belle fits, the $Z^{+}$amplitudes are coherent with those for the $K \pi^{+}$channel and, as in the case of the $Z(4430)^{+}$analysis, interference between the $Z^{+}$and $K \pi^{+}$amplitudes is very strong, in this case resulting in strong destructive interference below and above the peak region. A comparison of Figs. 10(a) and (b) clearly shows that by neglecting the possibility of interference between the $Z^{+}$and $K \pi^{+}$amplitudes, the BaBar analysis underestimates the strength of possible $Z_{1}^{+}$and $Z_{2}^{+}$signals in their data.

The $Z_{c}(3900)$ : The discovery and early measurements of the $Y(4260)$ were based on measurements of the initial state radiation process, $e^{+} e^{-} \rightarrow \gamma_{i s r} Y(4260)$ at $\sqrt{s} \simeq 10.6 \mathrm{GeV}$. This reaction requires that either the incident $e^{-}$or $e^{+}$radiates a $\sim 4.5 \mathrm{GeV}$ photon prior to annihilating, which results in a strong reduction in event rate. However, since the PEPII and KEKB $B$-factories ran with such high luminosity $\left(\mathcal{L}>10^{34} \mathrm{~cm}^{-2} \cdot \mathrm{s}^{-1}\right)$, the measurements were feasible. A more efficient way to produce $Y(4260)$ mesons would be to run a high luminosity $e^{+} e^{-}$collider as a "Y(4260) factory," i.e., at a cm energy of $4260 \mathrm{MeV}$, corresponding to the peak mass of the $Y(4260)$. This was done at the upgraded, two-ring Beijing electon-positron collider (BEPCII) [67] in 2013, and large numbers of $Y(4260)$ decays were detected in the new BESIII spectrometer [68].

The first channel to be studied with these data was $e^{+} e^{-} \rightarrow \pi^{+} \pi^{-} J / \psi$, where a distinct peak, called the $Z_{c}(3900)$, was seen near $3900 \mathrm{MeV}$ in the distribution of the larger of the two $\pi^{ \pm} J / \psi$ invariant mass combinations in each event $\left(M_{\max }(\pi J / \psi)\right)$, as can be seen in Fig. 11(a) [49]. A fit using a mass-independent-width BW function to represent the $\pi^{ \pm} J / \psi$ mass peak yielded a mass and width of $M_{Z_{c}(3900)}=3899.0 \pm 6.1 \mathrm{MeV}$ and $\Gamma_{Z_{c}(3900)}=46 \pm 22 \mathrm{MeV}$, which is $\sim 24 \mathrm{MeV}$ above the $m_{D^{*+}}+m_{\bar{D}^{0}}\left(\right.$ or $\left.m_{D^{+}}+m_{\bar{D}^{* 0}}\right)$ threshold. The $Z_{c}(3900)$ was observed by Belle in isr data at about the same time [50].

A subsequent BESIII study of the $\left(D \bar{D}^{*}\right)^{ \pm}$systems produced in $\left(D \bar{D}^{*}\right)^{ \pm} \pi^{\mp}$ final states in the same data sample found very strong near-threshold peaks in both the $D^{0} D^{*-}$ and $D^{+} \bar{D}^{* 0}$ invariant mass distributions [69], as shown in Figs. 11(b) and (c), respectively. The curves in the two figures show the results of fits to the data with threshold-modified BW line shapes to represent the peaks. The average values of the mass and widths from these fits are used to determine the resonance pole position $\left(M_{\text {pole }}+\mathrm{i} \Gamma_{\text {pole }}\right)$ with real and imaginary values of
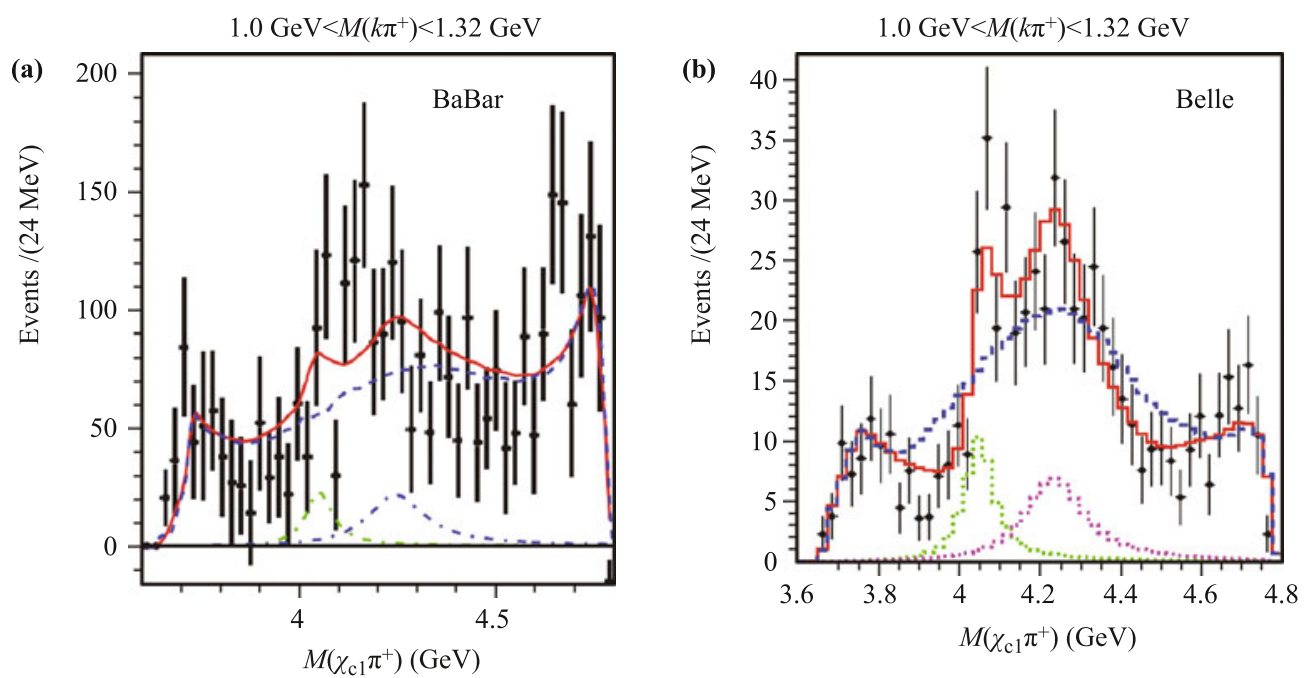

Fig. 10 (a) The data points show the background-subtracted $M^{2}\left(\pi^{+} \chi_{c 1}\right)$ distribution for $B \rightarrow K \pi^{+} \chi_{c 1}$ events with $1.0 \mathrm{GeV}<M\left(K \pi^{+}\right)<1.32 \mathrm{GeV}$ from BaBar. The dashed (blue) curve shows results from BaBar's fit with no $Z^{+}$ resonances; the red curve shows the results from a fit that includes two incoherent $Z^{+} \pi^{+} \chi_{c 1}$ resonances with Belle's fitted mass and width values. (b) The corresponding $M\left(\pi^{+} \chi_{c 1}\right)$ distribution from Belle. The solid red histogram is a projection Belle's fit with two coherent resonances in the $Z^{+} \rightarrow \pi^{+} \chi_{c 1}$ channel. The dashed (blue) curve indicates the best fit with no $Z^{+}$. 

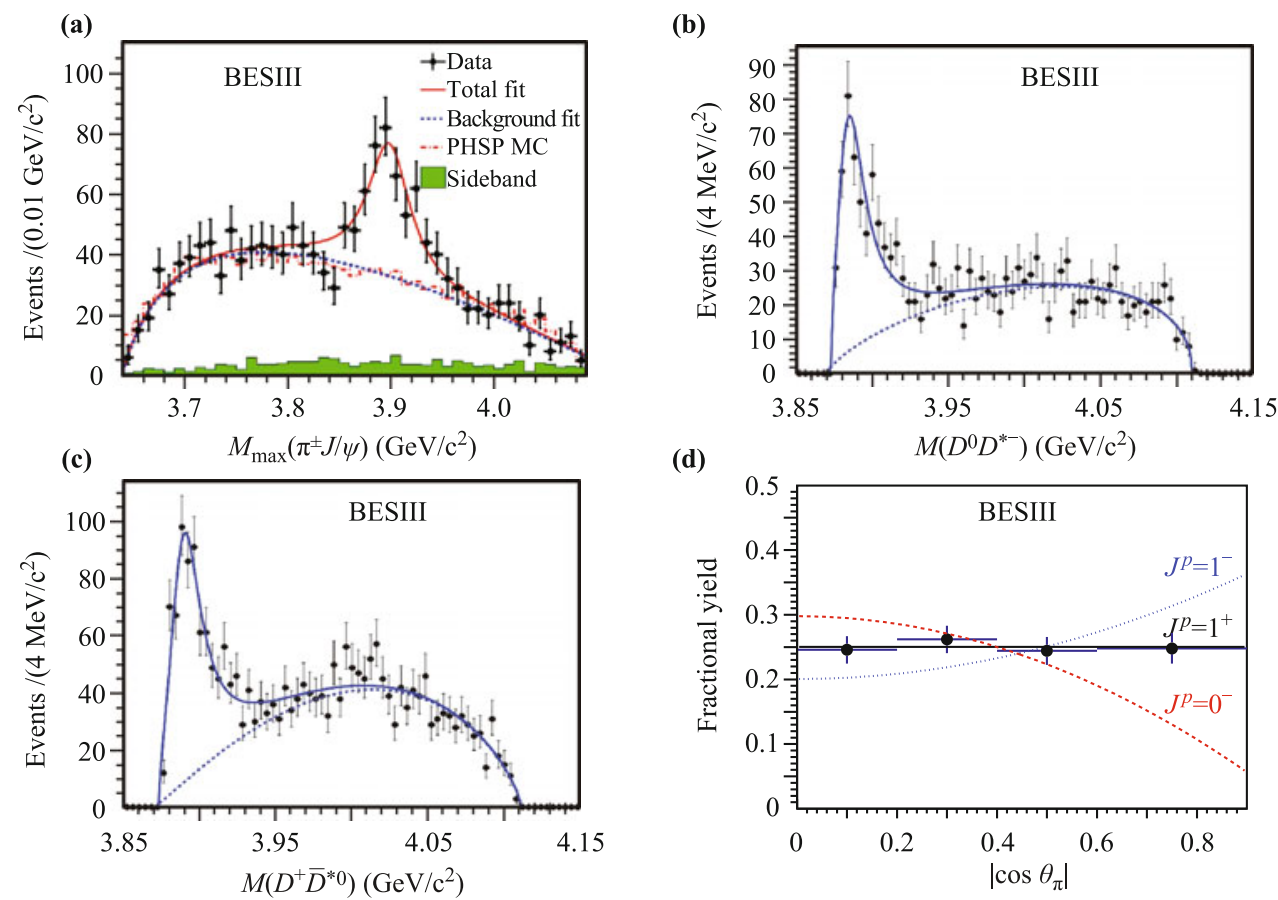

Fig. 11 (a) Invariant mass distributions for $\pi^{+} J / \psi$ from $e^{+} e^{-} \rightarrow \pi^{+} \pi^{-} J / \psi$ events from Ref. [49]; (b) $M\left(D^{0} D^{*-}\right)$ and (c) $M\left(D^{+} \bar{D}^{* 0}\right)$ for $e^{+} e^{-} \rightarrow\left(D \bar{D}^{*}\right)^{+} \pi^{-}$events from Ref. [69]; (d) The efficiency corrected production angle distribution compared with predictions for $J^{P}=0^{-}$(dashed-red), $J^{P}=1^{-}$(dotted blue) and $J^{P}=1^{+}$(solid black) quantum number assignments.

$M_{\text {pole }}=3883.9 \pm 4.5 \mathrm{MeV}$ and $\Gamma_{\text {pole }}=24.8 \pm 12 \mathrm{MeV}$.

Since the pole mass position is $\simeq 2 \sigma$ lower than the $Z_{c}(3900)$ mass reported in Ref. [49], BESIII cautiously named this $D \bar{D}^{*}$ state the $Z_{c}(3885)$. In the mass determinations of both the $Z_{c}(3885)$ and $Z_{c}(3900)$, effects of possible interference with a coherent component of the background are ignored, which can bias the measurements by amounts comparable to the resonance widths, and this might account for the different mass values. In any case, we consider it highly likely that the $Z_{c}(3885)$ is the $Z_{c}(3900)$ in a different decay channel. If this is the case, the partial width for $Z_{c}(3900) \rightarrow D \bar{D}^{*}$ decays is $6.2 \pm 2.9$ times larger than that for $J / \psi \pi^{+}$, which is small compared to open-charm vs. hidden-charm decaywidth ratios for established charmonium states above the open-charm threshold, such as the $\psi(3770)$ and $\psi(4040)$, where corresponding ratios are measured to be more than an order-of-magnitude larger [13].

Since the $Z(3885) \rightarrow D \bar{D}^{*}$ signals are so strong, the $J^{P}$ quantum numbers can be determined from the dependence of its production on $\theta_{\pi}$, the bachelor pion production angle relative to the beam direction in the $e^{+} e^{-} \mathrm{cm}$ system. For $J^{P}=0^{-}, \mathrm{d} N / \mathrm{d}\left|\cos \theta_{\pi}\right|$ should go as $\sin ^{2} \theta_{\pi}$; for $1^{-}$it should follow $1+\cos ^{2} \theta_{\pi}$ and for $1^{+}$it should be flat $\left(0^{+}\right.$is forbidden by Parity). Figure $11 \mathrm{~d}$ shows the efficiency-corrected $Z_{c}(3885)$ signal yield as a function of $\left|\cos \theta_{\pi}\right|$, together with expectations for $J^{P}=0^{+}$(dashed red), $1^{-}$(dotted blue) and $J^{P}=1^{+}$. The $J^{P}=1^{+}$as- signment is clearly preferred and the $0^{-}$and $1^{-}$assignments are ruled out with high confidence.

The $Z_{c}(4020)$ : With data accumulated at the peaks of the $Y(4260), Y(4360)$ and nearby energies, BESIII made a study of $\pi^{+} \pi^{-} h_{c}(1 P)$ final states. Exclusive $h_{c}(1 P)$ decays were detected via the $h_{c} \rightarrow \gamma \eta_{c}$ transition, where the $\eta_{c}$ was reconstructed in 16 exclusive hadronic decay modes. With these data, BESIII observed a distinct peak near $4020 \mathrm{MeV}$ in the $M_{\max }\left(\pi^{ \pm} h_{c}\right)$ distribution that is shown in Fig. 12(a). A fit to this peak, which the BESIII group called the $Z_{c}(4020)^{+}$, with a signal BW function (assuming $J^{P}=1^{+}$) plus a smooth background, returns a $\sim 9 \sigma$ significance signal with a fitted mass of $M_{Z_{c}(4020)}=4022.9 \pm 2.8 \mathrm{MeV}$, about $5 \mathrm{MeV}$ above $m_{D^{*+}}+m_{\bar{D}^{* 0}}$, and a width of $\Gamma_{Z_{c}(4020)}=7.9 \pm 3.7 \mathrm{MeV}[51]$. The product $\sigma\left(e^{+} e^{-} \rightarrow\right.$ $\left.\pi^{-} Z_{c}(4020)^{+}\right) \times \mathcal{B}\left(Z_{c}(4020)^{+} \rightarrow \pi^{+} h_{c}\right)$ is measured to be $7.4 \pm 2.7 \pm 1.2 \mathrm{pb}$ at $\sqrt{s}=4260 \mathrm{MeV}$, where the second error reflects the uncertainty of $\mathcal{B}\left(h_{c} \rightarrow \gamma \eta_{c}\right)$.

The inset in Fig. 12(a) shows the result of including a $Z_{c}(3900)^{+} \rightarrow \pi^{+} h_{c}$ term in the fit. In this case, a marginal $\sim 2 \sigma$ signal for $Z_{c}(3900)^{+} \rightarrow \pi^{+} h_{c}$ is seen to the left of the $Z_{c}(4020)$ peak. This translates into an upper limit on the product $\sigma\left(e^{+} e^{-} \rightarrow \pi^{-} Z_{c}(3900)^{+}\right) \times$ $\mathcal{B}\left(Z_{c}(3900)^{+} \rightarrow \pi^{+} h_{c}\right)$ of $11 \mathrm{pb}$. Since the product $\sigma\left(e^{+} e^{-} \rightarrow \pi^{-} Z_{c}(3900)^{+}\right) \times \mathcal{B}\left(Z_{c}(3900)^{+} \rightarrow \pi^{+} J / \psi\right)$ is measured to be $62.9 \pm 4.2 \mathrm{pb}$ [49], this limit implies that the $Z_{c}(3900)^{+} \rightarrow \pi^{+} h_{c}$ decay channel is suppressed 
(a)

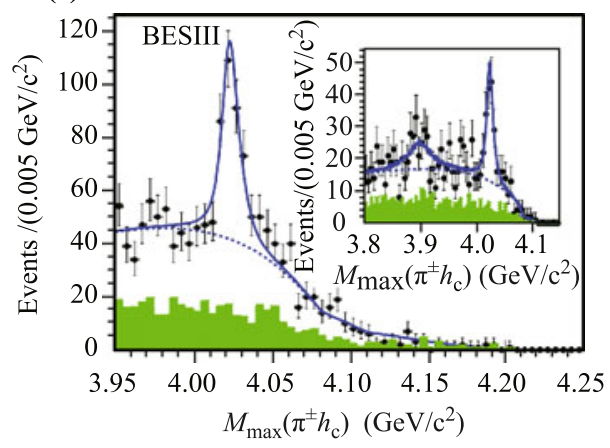

(c)

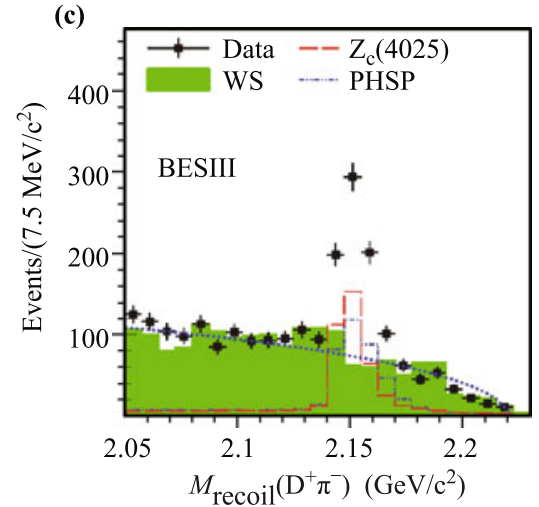

(b)

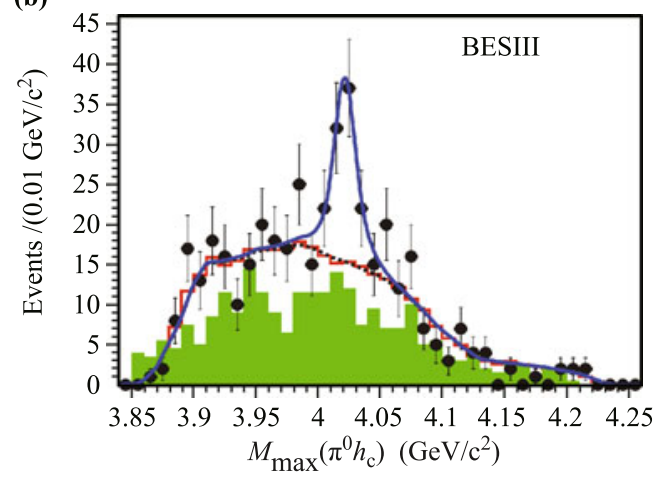

(d)

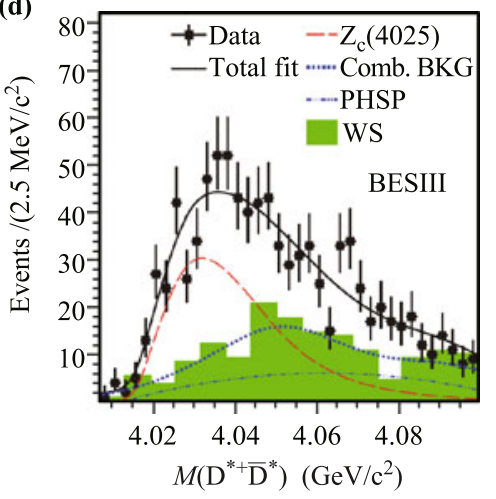

Fig. 12 (a) The $M_{\max }\left(\pi^{+} h_{c}\right)$ distribution for $e^{+} e^{-} \rightarrow \pi^{+} \pi^{-} h_{c}$ events from BESIII. The shaded histogram is background estimated from the $h_{c}$ mass sidebands. The curves are results of fits described in the text. (b) The corresponding $M_{\max }\left(\pi^{0} h_{c}\right)$ distribution for $e^{+} e^{-} \rightarrow \pi^{0} \pi^{0} h_{c}$ events from BESIII. (c) The distribution of masses recoiling from a detected $D^{+}$and $\pi^{-}$for $e^{+} e^{-} \rightarrow D^{+} \pi^{-} \pi^{0} X$ events at $\sqrt{s}=4260 \mathrm{MeV}$. The peak near $2.15 \mathrm{MeV}$ corresponds to $e^{+} e^{-} \rightarrow \pi^{-} D^{*+} \bar{D}^{* 0}$ events. The red dashed histogram shows the expected recoil mass distribution for $e^{+} e^{-} \rightarrow \pi^{-} Z_{c}$, with $M_{Z_{c}}=4025 \mathrm{GeV}$; the open, dash-dot histogram shows results for $\mathrm{MC} \pi^{-} D^{*+} \bar{D}^{* 0}$ three-body phase-space events. The shaded histogram is combinatoric background from wrong-sign combinations in the data. (d) $M\left(D^{*} \bar{D}^{*}\right)$ for $e^{+} e^{-} \rightarrow\left(D^{*} \bar{D}^{*}\right)^{+} \pi^{-}$events, i.e., events in the $2.25 \mathrm{MeV}$ peak in panel (c). The curves are described in the text.

relative to that for $\pi^{+} J / \psi$ by at least a factor of five.

BESIII recently reported observation of the neutral member of the $Z_{c}(4020)$ isospin triplet [70]. The $M_{\max }\left(\pi^{0} h_{c}\right)$ distribution for $e^{+} e^{-} \rightarrow \pi^{0} \pi^{0} h_{c}$ events in the same data set used for the $Z_{c}(4020)^{ \pm}$, shown in Fig. 12 (b), looks qualitatively like the $M_{\max }\left(\pi^{+} h_{c}\right)$ distribution with a distinct peak near $4020 \mathrm{MeV}$. A fit to the data that includes a BW term with a width fixed at the value measured for the $Z_{c}(4020)^{+}$and floating mass returns a mass of $4023.9 \pm 4.4 \mathrm{MeV}$; this and the signal yield are in good agreement with expectations based on isospin symmetry.

A study of $e^{+} e^{-} \rightarrow D^{*+} \bar{D}^{* 0} \pi^{-}$events in the $\sqrt{s}=$ $4.26 \mathrm{GeV}$ data sample using a partial reconstruction technique that only required the detection of the bachelor $\pi^{-}$, the $D^{+}$from the $D^{*+} \rightarrow \pi^{0} D^{+}$decay and one $\pi^{0}$, either from the $D^{*+}$ or the $\bar{D}^{* 0}$ decay, to isolate the process and measure the $D^{*+} \bar{D}^{* 0}$ invariant mass [52]. The signal for real $D^{*+} \bar{D}^{* 0} \pi^{-}$final states is the distinct peak near $2.15 \mathrm{MeV}$ in the $D^{+} \pi^{-}$recoil mass spectrum shown on Fig. 12(c). The measured $D^{*} \bar{D}^{*}$ invariant mass distribution for events in the $2.15 \mathrm{MeV}$ peak, shown as data points in Fig. 12(d), shows a strong nearthreshold peaking behavior with a shape that cannot be described by a phase-space-like distribution, shown as a dash-dot blue curve, or by combinatoric background, which is determined from wrong-sign (WS) events in the data (i.e., events where the bachelor pion and charged $D$ meson have the same sign) that are shown as the shaded histogram. The solid black curve shows the results of a fit to the data points that includes an efficiency weighted $S$-wave BW function, the WS background shape scaled to measured non- $D^{*+} \bar{D}^{* 0} \pi^{-}$background level under the signal peak in Fig. 12(d), and a phase-space term. The fit returns a $13 \sigma$ signal with mass and width $M=4026.3 \pm 4.5 \mathrm{MeV}$ and $\Gamma=24.8 \pm 9.5$ $\mathrm{MeV}$, values that are close to those measured for the $Z_{c}(4020)^{+} \rightarrow \pi^{+} h_{c}$ channel. Although BESIII cautiously calls this $\left(D^{*} \bar{D}^{*}\right)^{+}$signal the $Z_{c}(4025)$, in the following we assume that this is another decay mode of the $Z_{c}(4020)$.

From the numbers provided in Ref. [52], we determine $\sigma\left(e^{+} e^{-} \rightarrow \pi^{-} Z_{c}(4020)\right) \times \mathcal{B}\left(Z_{c}(4020) \rightarrow D^{*} \bar{D}^{*}\right)=$ $89 \pm 19 \mathrm{pb}$. This implies that the partial width for 
$Z_{C}(4020) \rightarrow D^{*} \bar{D}^{*}$ is larger than that for $Z_{c}(4020) \rightarrow$ $\pi h_{c}$, but only by a factor of $12 \pm 5$, not by the large factors that are characteristic of open charm decays of conventional charmonium.

There is no sign of $Z_{c}(4020) \rightarrow D \bar{D}^{*}$ in either Figs. 11(b) or (c), where $\sim 500$ and $\sim 700$ event $Z_{c}(3885)$ signals in the $D^{0} D^{*-}$ and $D^{+} \bar{D}^{* 0}$ distributions, respectively, correspond to the product $\sigma\left(e^{+} e^{-} \rightarrow\right.$ $\left.\pi^{-} Z_{c}(3900)\right) \times \mathcal{B}\left(Z_{c}(3900) \rightarrow D \bar{D}^{*}\right)=84 \pm 23 \mathrm{pb}$, about the same as the corresponding product for the $Z_{c}(4020) D^{*} \bar{D}^{*}$ signal mentioned in the previous paragraph. This absence of any evident signals for $Z_{c}(4020) \rightarrow D \bar{D}^{*}$ in Figs. 11(b) and (c) suggests that the $Z_{c}(4020) \rightarrow D \bar{D}^{*}$ partial width is considerably smaller than that for $Z_{c}(4020) \rightarrow D^{*} \bar{D}^{*}$, which may have some relation to the $Z_{c}(4020)$ 's proximity to the $2 m_{D^{*}}$ threshold. However, until BESIII provides a limit on a $Z_{c}(4020) \rightarrow D \bar{D}^{*}$ signal at $\sqrt{s}=4260 \mathrm{MeV}$, this effect cannot be quantified.

$\boldsymbol{X} \mathbf{( 3 9 1 5 )}$ The $X(3915)$ is an $\omega J / \psi$ mass peak with $M=3918 \pm 2 \mathrm{MeV}$ with $\Gamma=20 \pm 5 \mathrm{MeV}$ [13] seen in $B \rightarrow K \omega J / \psi$ decays by both Belle [71] and BaBar [72, 73] [see Figs. 13(a) and (b)] and in the two-photon process $\gamma \gamma \rightarrow \omega J / \psi$ also by Belle [74] and BaBar [75] [Fig. $13(\mathrm{c})]^{8)}$. BaBar measured its $J^{P C}$ quantum numbers to be $0^{++}$. The PDG currently assigns this as the $\chi_{c 0}(2 P)$ charmonium level (i.e., the $\chi_{c 0}^{\prime}$ ), an assignment that has a number of problems [77]:

i) The $J=2$ member of the $\chi_{c 0,1,2}^{\prime}$ multiplet, the $\chi_{c 2}(2 P)\left(\chi_{c 2}^{\prime}\right)$, was seen by Belle [78] and confirmed by
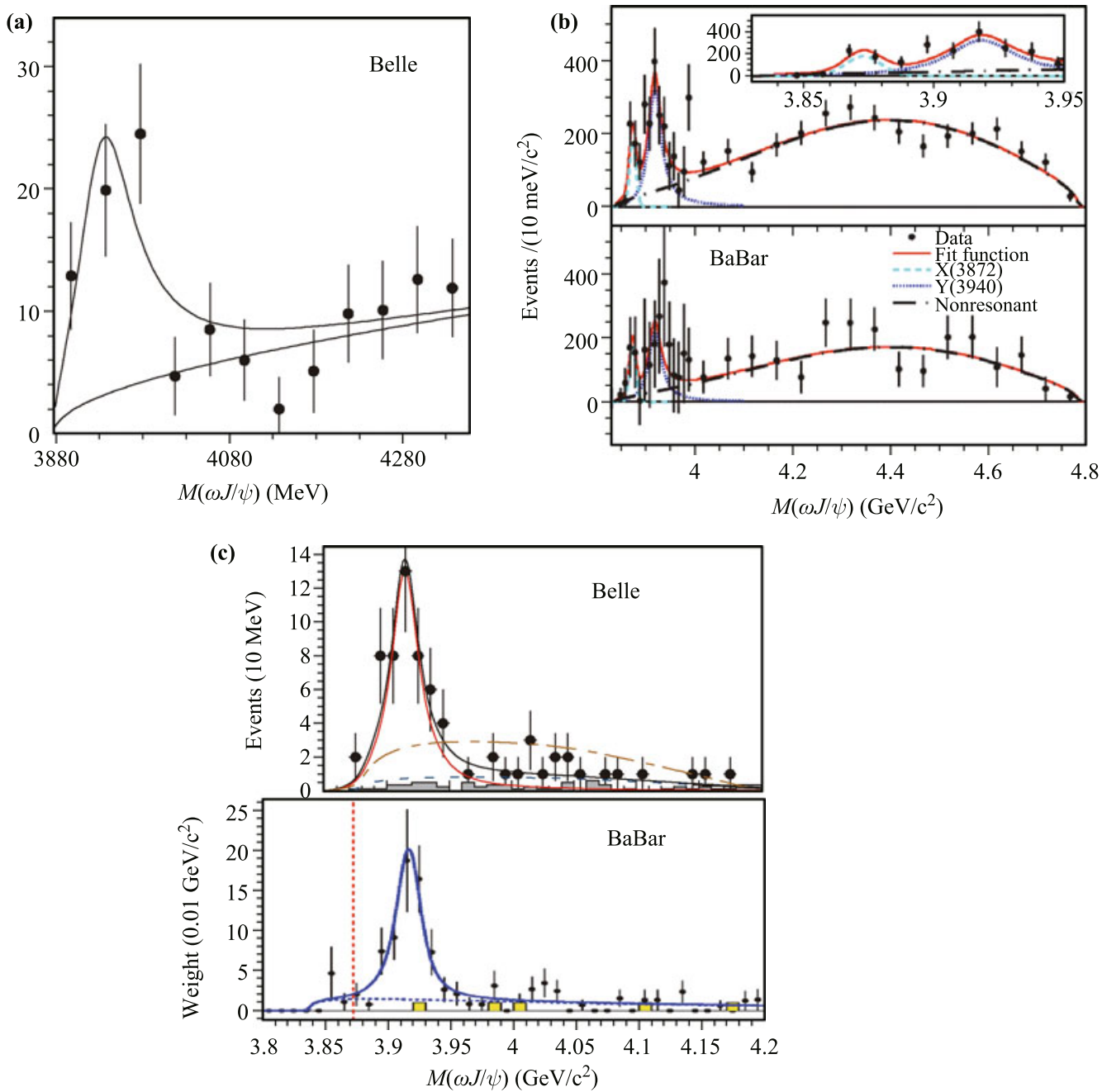

Fig. 13 (a) The $X(3915) \rightarrow \omega J / \psi$ signals in $B \rightarrow K \omega J / \psi$ decays from Belle. (b) The corresponding distributions from BaBar, where the upper panel shows results for $B^{+} \rightarrow K^{+} \omega J / \psi$ and the lower panel shows those for $B^{0} \rightarrow K_{S} \omega J / \psi$. The inset in the upper panel shows an expanded view of the low end of the $\omega J / \psi$ mass scale, where the smaller, low-mass peak is due the $X(3872) \rightarrow \omega J / \psi$ and the larger, higher mass peak is the $X(3915) \rightarrow \omega J / \psi$ signal. (c) The $X(3915) \rightarrow \omega J / \psi$ signals in $\gamma \gamma \rightarrow \omega J / \psi$ fusion reactions from Belle (upper) and BaBar (lower).

\footnotetext{
8) Some of the literature refers to this state as the $Y(3940)$.
} 
BaBar [79] as a distinct peak in the $D \bar{D}$ invariant mass distribution near $3930 \mathrm{MeV}$ in $\gamma \gamma \rightarrow D \bar{D}$ events. The mass, production rate and production angle dependence agree quite well with expectations for the $\chi_{c 2}^{\prime}$ [see Figs. 14(a) and (b)], and there are no known reasons to doubt this assignment. The weighted averages of the Belle and BaBar mass and width measurements are $M=3927 \pm 3$ $\mathrm{MeV}$ and $\Gamma=24 \pm 6 \mathrm{MeV}$ [13]. With the $\chi_{c 2}^{\prime}$ well established with a reliably known mass, the $X(3915)=\chi_{c 0}^{\prime}$ assignment implies a $2^{3} P_{2}-2^{3} P_{0}$ fine splitting of only $9 \pm 4 \mathrm{MeV}$, which is very small in comparison with the corresponding $n=1$ splitting of $141.4 \pm 0.3 \mathrm{MeV}$ [13] and theoretical predictions for $n=2$ that range from 69 $\mathrm{MeV}$ [36] to $80 \mathrm{MeV}$ [76].

ii) There is no sign of $X(3915) \rightarrow D \bar{D}$ decay, which is expected to be a favored decay mode. Belle [80] and BaBar [81] have studied the process $B \rightarrow K D \bar{D}$ and both groups see prominent signals for $\psi(3770) \rightarrow D \bar{D}$, but no hint of a $D \bar{D}$ mass peak near $3915 \mathrm{MeV}$ [see Fig. 14(c)]. Since neither group reported a $X(3915) \rightarrow D \bar{D}$ limit, I formed my own conservative estimate of an upper bound by scaling the total number of Belle events in the two mass bins surrounding $3915 \mathrm{MeV}$ in the upper panel of Fig. 14(c) to the $\psi(3770)$ signal while assuming constant acceptance. This gives $\mathcal{B}(X(3915) \rightarrow$ $\omega J / \psi)>\mathcal{B}\left(X(3915) \rightarrow D^{0} \bar{D}^{0}\right)$, which strongly contradicts the theoretical expectation that the $\chi_{c 0}^{\prime} \rightarrow D \bar{D}$ decay channel should dominant [76].

iii) Since branching fractions cannot exceed unity, the measured quantity $\Gamma_{\gamma \gamma}^{\chi_{c 2}^{\prime}} \times \mathcal{B}\left(\chi_{c 2}^{\prime} \rightarrow D \bar{D}\right)=$ $0.21 \pm 0.04 \mathrm{keV}[78,79]$, translates to a $\sim 90 \% \mathrm{CL}$ lower limit $\Gamma_{\gamma \gamma}^{\chi_{c 2}^{\prime}}>0.16 \mathrm{keV}$. Using the assumption $\Gamma_{\gamma \gamma}^{\chi_{c 0}^{\prime}} / \Gamma_{\gamma \gamma}^{\chi_{c 2}^{\prime}}=\Gamma_{\gamma \gamma}^{\chi_{c 0}} / \Gamma_{\gamma \gamma}^{\chi_{c 2}}=4.5 \pm 0.6$ [13], which is valid for pure charmonium states, I infer a lower limit $\Gamma_{\gamma \gamma}^{\chi_{c 0}^{\prime}}>0.80 \mathrm{keV}$ that, when taken with the measured value $\Gamma_{\gamma \gamma}^{X(3915)} \mathcal{B}(X(3915) \rightarrow \omega J / \psi)=0.054 \pm 0.009$ $\mathrm{keV}$ [82], gives an upper limit $\mathcal{B}\left(\chi_{c 0}^{\prime} \rightarrow \omega J / \psi\right)<5.6 \%$ if

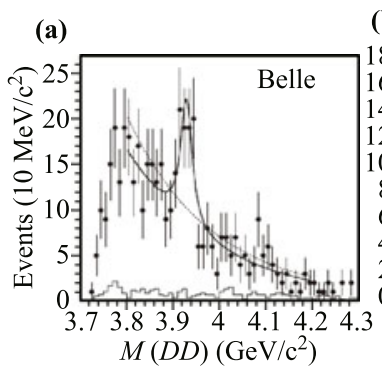

(b)

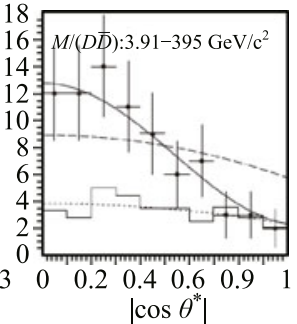

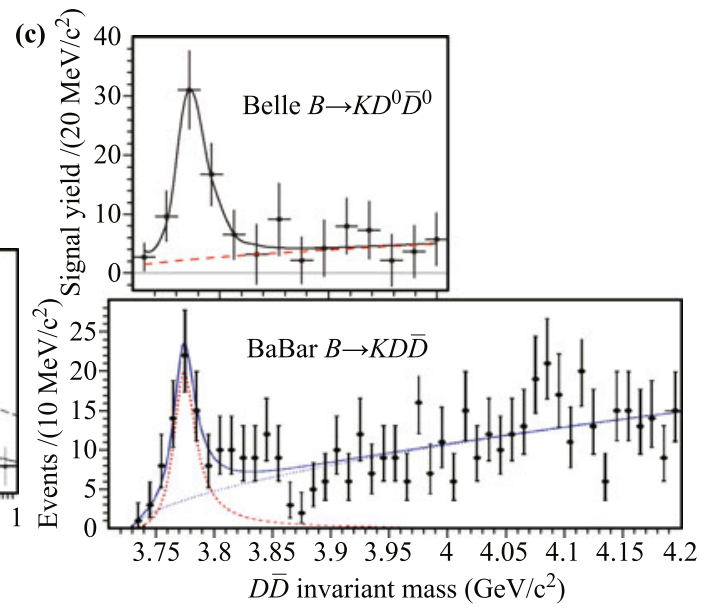

(d)

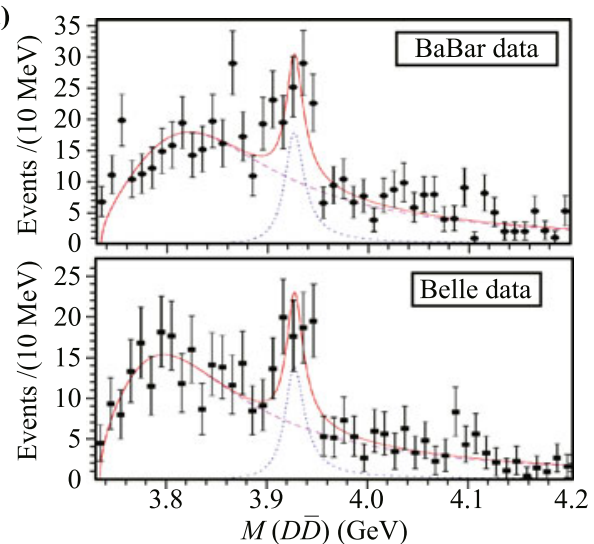

Fig. 14 (a) The $D \bar{D}$ invariant mass distribution for $\gamma \gamma \rightarrow D \bar{D}$ events in Belle. The narrow peak is attributed to $\chi_{c 2}^{\prime} \rightarrow D \bar{D}$. (b) The $\left|\cos \theta^{*}\right|$ distribution for events in the region of the $\chi_{c 2}^{\prime}$ peak. The solid line show expectations for spin=2, helicity=2; the dashed line shows the spin zero expectation. (c) The $M\left(D^{0} \bar{D}^{0}\right)$ distribution from $B \rightarrow K D^{0} \bar{D}^{0}$ decays from Belle [80] (upper); the $M(D \bar{D})$ distribution from $B \rightarrow K D \bar{D}$ decays from BaBar [81] (lower). The near-threshold peak in both plots is due to the $\psi(3770)$. (d) The $M(D \bar{D})$ distribution from $\gamma \gamma \rightarrow D \bar{D}$ two-photon annihilation from BaBar [79] (upper); the same distribution from Belle [78] (lower). The narrow peak is due to the $\gamma \gamma \rightarrow \chi_{c 2}^{\prime} \rightarrow D \bar{D}$ and the curves show results of fits described in Ref. [77]. 
$X(3915)=\chi_{c 0}^{\prime}$. The PDG value for the $X(3915)$ total width of $20 \pm 5 \mathrm{MeV}$ and the above-mentioned limit $\mathcal{B}(X(3915) \rightarrow D \bar{D})<\mathcal{B}(X(3915) \rightarrow \omega J / \psi)$ imply an upper limit $\Gamma\left(\chi_{c 0}^{\prime} \rightarrow D \bar{D}\right)<1.5 \mathrm{MeV}$ for the $X(3915)=$ $\chi_{c 0}^{\prime}$ assignment. This upper limit is below the range of theoretical predictions that range from $7 \mathrm{MeV}$ [83] to 187 $\mathrm{MeV}$ [84].

iv) The average of the Belle and BaBar measurements for $X(3915)$ production in $B$ meson decay is $\mathcal{B}\left(B \rightarrow K X_{3915}\right) \times \mathcal{B}\left(X_{3915} \rightarrow \omega J / \psi\right)=\left(3.0_{-0.7}^{+0.9}\right) \times 10^{-5}$. If I make the (reasonable?) assumption that $\mathcal{B}(B \rightarrow$ $\left.K \chi_{c 0}^{\prime}\right)<\mathcal{B}\left(B \rightarrow K \chi_{c 0}\right)=(1.3 \pm 0.2) \times 10^{-4}[13], \mathrm{I}$ find the lower limit $\mathcal{B}\left(\chi_{c 0}^{\prime} \rightarrow \omega J / \psi\right)>14 \%$, in strong contradiction to the $5.6 \%$ upper limit established in the previous paragraph.

Some authors have identified another candidate for the $\chi_{c 0}^{\prime}$. Guo and Meissner [77] propose that the broad background under the $\gamma \gamma \rightarrow \chi_{c 2}^{\prime} \rightarrow D \bar{D}$ peak in the $M(D \bar{D})$ distribution in $\gamma \gamma \rightarrow D \bar{D}$ events as seen in Fig. 14(a) is due to $\gamma \gamma \rightarrow \chi_{c 0}^{\prime} \rightarrow D \bar{D}$. Their fits to BaBar and Belle data, shown as dashed curves Fig. $14(\mathrm{~d})$, yield a " $\chi_{c 0}^{\prime}$ " mass and width of $M=3838 \pm 12 \mathrm{MeV}$ and $\Gamma=221 \pm 21$ $\mathrm{MeV}$, which is in general agreement with charmonium model expectations. This fit is almost certainly biased because the authors do not consider contributions to the $D \bar{D}$ signal from $\chi_{c 2}^{\prime} \rightarrow D \bar{D}^{*}$ decays where the $\pi$ or $\gamma$ from $\bar{D}^{*} \rightarrow \bar{D} \pi(\gamma)$ decay is missed. Nevertheless, their suggestion that a broad and light $\chi_{c 0}^{\prime}$ might be responsible for at least part of the measured $D \bar{D}$ "background" should be carefully considered. With enough data, contributions from $\chi_{c 0}^{\prime} \rightarrow D \bar{D}$ and $\chi_{c 2} \rightarrow D \bar{D}^{*}$ can be sorted out from the relative rates for $D^{0} \bar{D}^{0}, D^{*+} D^{*-}$ and $D^{0} D^{*-}$ final states and the shapes of their associated $p_{T}$ distributions. This is a good candidate for a "day one" measurement for the BelleII experiment [85].

Chao [33] suggested that $\chi_{c 0}^{\prime} \rightarrow D \bar{D}$ may be the source of the broad peak in the $M(D \bar{D})$ spectrum seen by Belle in $e^{+} e^{-} \rightarrow J / \psi D \bar{D}$ events [32], as discussed above and shown in the upper panel of Fig. 5(b). The Belle group's fit to this distribution yielded a broad signal with a peak mass at $3780 \pm 48 \mathrm{MeV}$ and a significance of $\simeq 3.8 \sigma$. This mass is closer to expectations for the $\chi_{c 0}^{\prime}$ than that of the $X(3915)$. Belle is now investigating this reaction with about twice as much data than was used in the Ref. [32] analysis.

Although these suggestions are not definitive, they, plus the arguments from the previous paragraph, show that the PDG's assignment of the $X(3915)$ to the $\chi_{c 0}^{\prime}$ charmonium level is premature and possibly misleading.

If the $X(3915)$ is not an ordinary $c \bar{c}$ charmonium state, what is it? Molina and Oset proposed a molecule-like picture with states that are dynamically generated from vector-vector interactions. They find a $J^{P C}=0^{++}$state near $3940 \mathrm{MeV}$ with a $17 \mathrm{MeV}$ width [86] that is dominantly $D^{*} \bar{D}^{*}$ and $D_{s}^{*} D_{s}^{*}$. The $X(3915)$ mass is below the threshold for decays to these final states and the authors find a strong decay to $\omega J / \psi$ plus a strong suppression of decays to $D \bar{D}$, consistent with what is seen experimentally. This paper also predicts other states, including some with isospin=1, but none of these can be as easily associated with any of the $X Y Z$ peaks that are seen in experiment.

$\boldsymbol{Y}(\mathbf{4 1 4 0 )}$ In 2009, the CDF group reported $3.8 \sigma \mathrm{ev-}$ idence for a near-threshold peak in the $\phi J / \psi$ invariant mass distribution from $B^{+} \rightarrow K^{+} \phi J / \psi$ decays based on an analysis of a $2.7 \mathrm{fb}^{-1}$ data sample [87]. CDF subsequently reproduced this signal with a larger, 6.0 $\mathrm{fb}^{-1}$ data sample and with a significance that increased to more than $5 \sigma$ [88] [see Fig. 15(a)]. The mass and width were reported as $M=4143.4 \pm 3.0 \mathrm{MeV}$ and $\Gamma=15.3_{-6.6}^{+10.7} \mathrm{MeV}$. The CDF group's fit, shown as a red curve in Fig. 15(a), includes a second structure with mass and width $M=4274.4_{-7.0}^{+8.6} \mathrm{MeV}$ and $\Gamma=32.3_{-17.1}^{+23.2}$ $\mathrm{MeV}$, with a significance estimated to be $3.1 \sigma$.

The existence of the $Y(4140)$ has been somewhat controversial because it was not seen in $B \rightarrow K \phi J / \psi$ decays by LHCb [89], which set an upper limit on the $Y(4140)$ production rate in $B$ decays that is about a factor of two below the CDF group's central value for the same quantity. Figure 15(b) shows the LHCb group's $M(\phi J / \psi)$ distribution as a histogram with expectations for the two peaks reported by CDF superimposed as blue dashed curves. While the agreement between the LHCb data and the CDF-inspired curves is pretty poor, there does seem to be excesses in the $\mathrm{LHCb}$ distribution in the vicinity of both of the CDF peaks. The excess near threshold is pretty broad and suggests that if there is a state decaying to $\phi J / \psi$ in that mass region, it may have a broader width than the CDF group's measured $\Gamma$ value.

This year, the CMS group published the $M(\phi J / \psi)$ distribution in $B^{+} \rightarrow K^{+} \phi J / \psi$ shown in Fig. $15(\mathrm{c})$, where there are distinct signals near the positions of both of the CDF peaks [90]. Their fit to the data, shown as red curves in the figure, give a $Y(4140)$ mass an width of $M=4148.0 \pm 6.7 \mathrm{MeV}$ and $\Gamma=28_{-22}^{+24} \mathrm{MeV}$, which agree within errors with the CDF measurements. The estimated significance of the $Y(4140)$ peak is greater than $5 \sigma$. Note that the central value of the CMS width measurement, while consistent with the CDF value, is higher by nearly a factor of two, and has a large positive-side error.

The fitted mass of the second peak, $M=4314 \pm 9$ $\mathrm{MeV}$, is about $3 \sigma$ higher than the CDF value, while the fitted width, $\Gamma=38_{-22}^{+34} \mathrm{MeV}$, is in good agreement, 


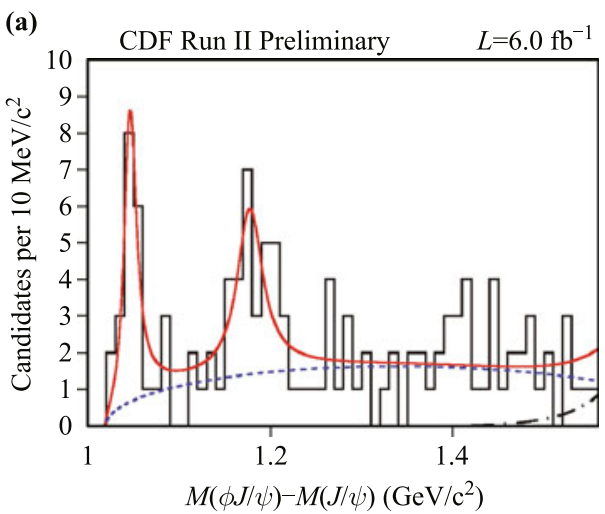

(b)
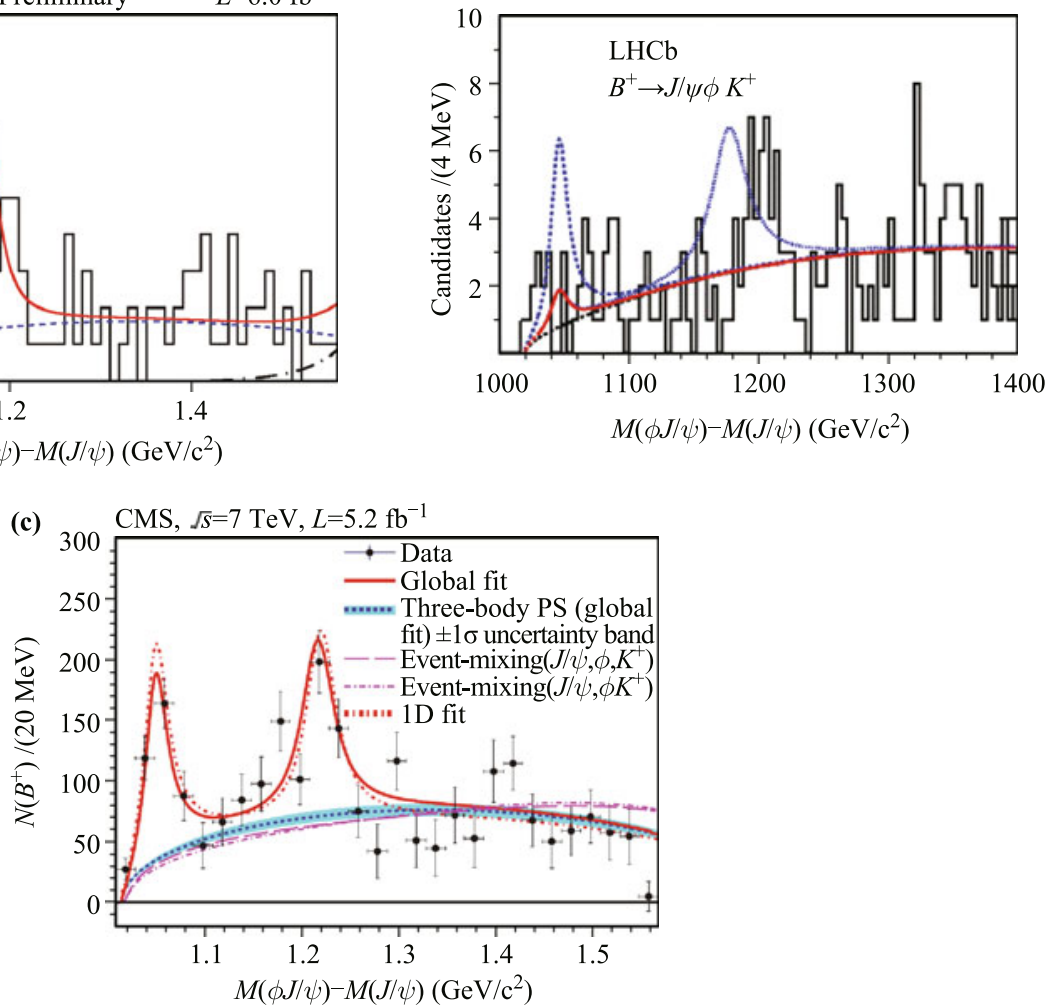

Fig. 15 (a) The CDF $6.0 \mathrm{fb}^{-1} M(\phi J / \psi)-M(J / \psi)$ distribution for $B^{+} \rightarrow K^{+} \phi J / \psi$ decays is shown as a histogram. The red curve shows the results of a fit that includes the $Y(4140)$, a possible second resonance near $4275 \mathrm{MeV}$ and a phase-space non-resonant term (shown as a dashed line). (b) The same distribution from LHCb, with expectations based on the CDF results shown as a dashed blue curve. The LHCb upper limit is based on the results of the fit to their data shown as a solid red curve. (c) The same distribution from the CMS group with the results of their fits superimposed.

albeit with large errors. The CMS paper also provides a plot that compares the $K^{+} K^{-} K^{+}$invariant mass distribution for $B \rightarrow K^{+} K^{-} K^{+} J / \psi$ decays with phase-space expectations [see Fig. 16(a)], where there is some indication of structure around $1.7 \sim 1.8 \mathrm{GeV}$. The CMS group warns that if such a structure actually exists, it could create a reflection peak in the $M\left(K^{+} K^{-} J / \psi\right)$ distribution near $4.3 \mathrm{GeV}$ that could distort the measured parameters of the second $\phi J / \psi$ mass peak. As was the case for the $Z(4430)$ parameter extraction from $B \rightarrow K \pi^{+} \psi^{\prime}$ decays, a better understanding of this second peak probably needs an experiment with a data sample that is large enough to support an amplitude analysis.

The BaBar group recently reported on a search for the $Y(4140)$ in $B \rightarrow K \phi J / \psi$ decays [91]. Figure 16(b) shows their efficiency-corrected $M(\phi J / \psi)$ distribution together with results of a fit that includes two resonances with the CDF group's mass and width values. Although there are signs of signals both for the $Y(4140)$ and the $Y(4275)$, the statistical significance in each case is less that $2 \sigma$ after systematic errors are considered.

The $Y(4140)$ shows up just above the $m_{\phi}+m_{J / \psi}$ mass threshold and e.g., the $X(3915)$ mass is just above the $m_{\omega}+m_{J / \psi}$ mass threshold. This led to some speculation 101401-18 that the two states may be related: e.g., the $X(3915)$ may be a $D^{*} \bar{D}^{*}$ molecule and the $Y(4140)$ is its hidden charm counterpart $D_{s}^{*+} D_{s}^{*-}$ molecule $[92,93]$. In this case the $Y(4140)$ would have the same $J^{P C}$ quantum numbers as the $X(3915)$, namely $0^{++}$, and this implies that the $Y(4140)$ could be accessible in $\gamma \gamma$ fusion reactions. The $M(\phi J / \psi)$ distribution from a Belle study of $\gamma \gamma \rightarrow \phi J / \psi$ near the $m_{\phi}+m_{J / \psi}$ threshold is shown in Fig. 16(c), where there is no sign of the $Y(4140)$ but $\sim 3 \sigma$ evidence for a peak at $M=4350.6_{-5.1}^{+4.6}$ $\mathrm{MeV}$ with width $\Gamma=13_{-10}^{+18} \mathrm{MeV}$ [94]. The mass value is $\sim 4 \sigma$ above that of the second peak seen by CDF and CMS in $B \rightarrow K \phi J / \psi$ decays and, so, if both observations are from real meson states, they are not likely to be from the same source. The absence of any events in the $Y(4140)$ region translates into the upper limit $\Gamma_{\gamma \gamma}^{Y(4140)} \times \mathcal{B}(Y(4140) \rightarrow \phi J / \psi)<41 \mathrm{eV}$ for $J^{P C}=0^{++}$, which is smaller, but not much smaller, than the measured value $(54 \pm 9 \mathrm{eV})$ of the corresponding quantity for the $\gamma \gamma \rightarrow X(3915) \rightarrow \omega J / \psi$ signal discussed above. However, it is much lower than theoretical expectations for a $D_{s}^{*+} D_{s}^{*-}$ molecule: $176_{-93}^{+137} \mathrm{eV}$ for $J^{P C}=0^{++}$or $189_{-100}^{+147^{s}} \mathrm{eV}$ for $J^{P C}=2^{++}[94]$.

Stephen Lars Olsen, Front. Phys. 10, 101401 (2015) 

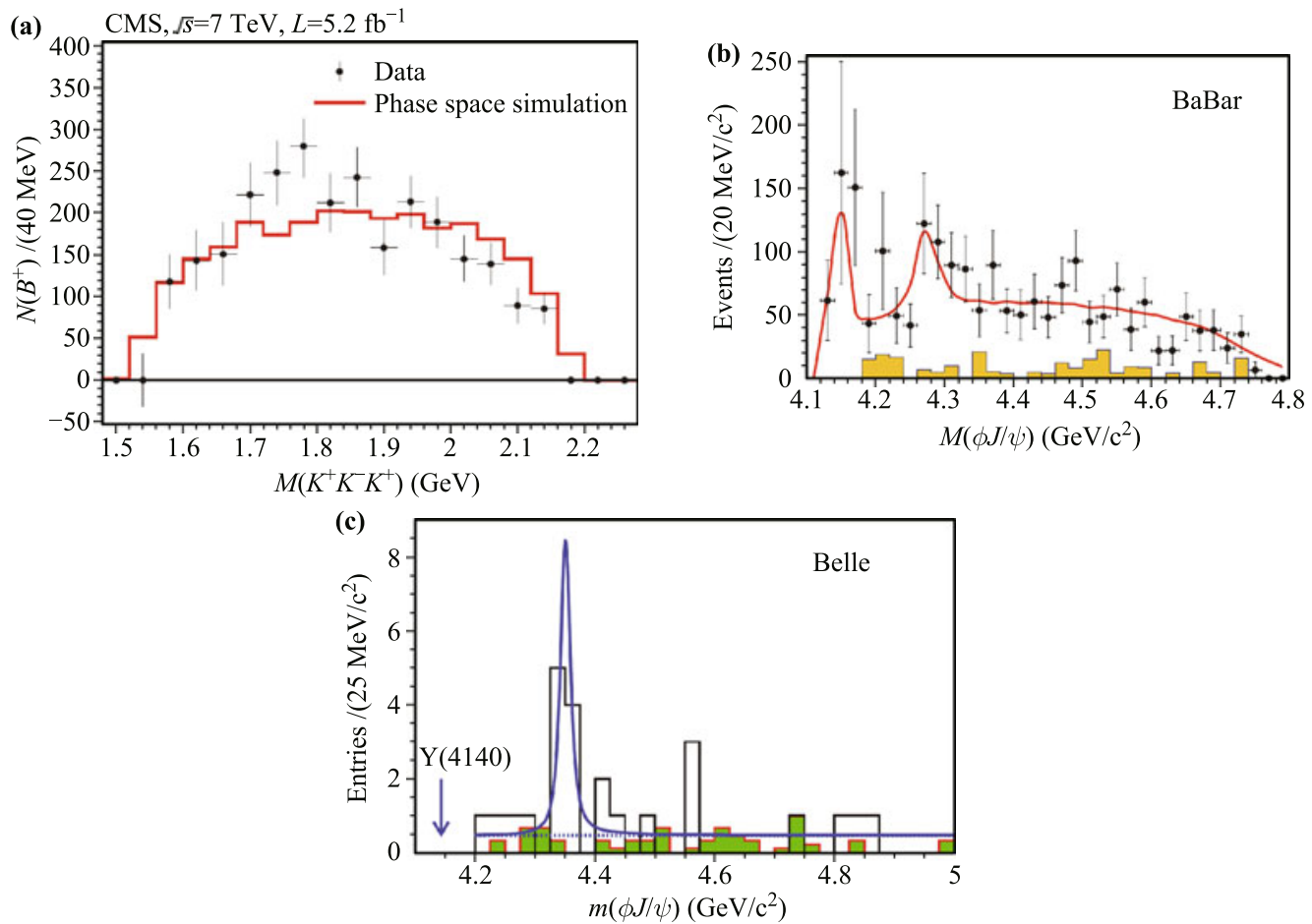

Fig. 16 (a) The data points show the CMS experiment's $M\left(K^{+} K^{-} K^{+}\right)$distribution from $B^{+} \rightarrow K^{+} K^{-} K^{+} J / \psi$ decays; the histogram shows the expectations for a four-body phase-space distribution. (b) The efficiency corrected $M(\phi J / \psi)$ distribution for $B \rightarrow K \phi J / \psi$ decays from BaBar. The red curve shows the results of a fit that includes two resonances with mass and width fixed at the CDF values. (c) The open histogram shows the $M(\phi J / \psi)$ distribution for $\gamma \gamma \rightarrow \phi J / \psi$ events from Belle; the shaded histogram shows the background estimated from the $\phi$ and $J / \psi$ mass sidebands. The solid curve shows the results of the fit described in the text.

The $\mathrm{LHCb}$ results discussed here were based on an analysis of a $0.17 \mathrm{fb}^{-1}$ data sample, which is only about one tenth of their current total. Hopefully new results based on all of the available data will soon be available.

$\boldsymbol{X} \mathbf{( 3 8 7 2 )}$ The $X(3872)$ was first seen in 2003 as a peak in the $\pi^{+} \pi^{-} J / \psi$ invariant mass distribution in $B \rightarrow K \pi^{+} \pi^{-} J / \psi$ decays [95] as shown in Fig. 17(a). It is well established and has been seen and studied by a number of experiments [96-100]. The PDG average mass value, $3871.68 \pm 0.17 \mathrm{MeV}$, is indistinguishable from that for the $m_{D^{0}}+m_{D^{* 0}}=3871.84 \pm 0.27 \mathrm{MeV}$ threshold [13]; a recent result from $\mathrm{LHCb}$ is $M_{X(3872)}-\left(m_{D^{0}}+m_{D^{* 0}}\right)=$ $-0.09 \pm 0.28 \mathrm{MeV}$ [101]. It is also narrow, Belle has reported a $95 \%$ C.L. upper limit on its total width of $\Gamma<1.2 \mathrm{MeV}[102]$.

While $\mathrm{LHCb}$ and CDF have conclusively established the $J^{P C}$ quantum numbers as $1^{++}[103,104]$, its isospin is less well understood. Both CDF [105] and Belle [102]
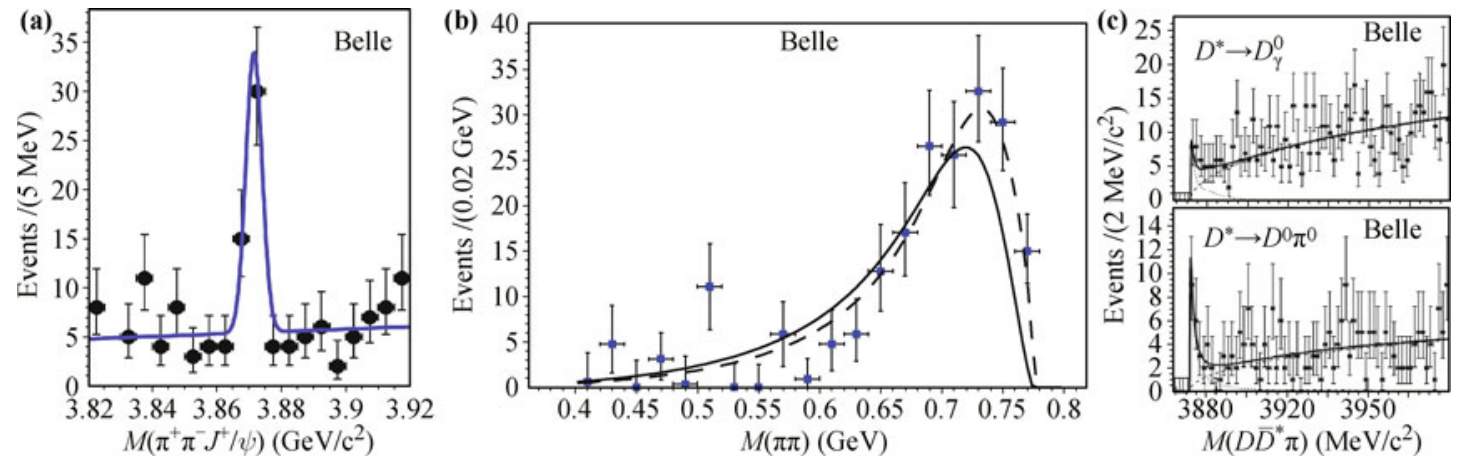

Fig. 17 (a) The $\pi^{+} \pi^{-} J / \psi$ invariant mass distribution for $B \rightarrow K \pi^{+} \pi^{-} J / \psi$ events from Belle's original $X(3872)$ paper. (b) The $\pi^{+} \pi^{-}$invariant mass distribution for $X(3872) \rightarrow \pi^{+} \pi^{-} J / \psi$ events in Belle. The curves shows results of fits to a $\rho \rightarrow \pi^{+} \pi^{-}$line shape including $\rho$ - $\omega$ interference. The dashed (solid) curve is for even (odd) $X(3872)$ parity. (c) $M\left(D^{0} \bar{D}^{* 0}\right)$ distributions from $B \rightarrow K D^{0} \bar{D}^{* 0}$ decays. The upper plot is for $\bar{D}^{* 0} \rightarrow \bar{D}^{0} \gamma$ decays, the lower plot is for $\bar{D}^{* 0} \rightarrow \bar{D}^{0} \pi^{0}$ decays. The peaks near threshold are attributed to $X(3872) \rightarrow D^{0} \bar{D}^{* 0}$ decays. 
have shown that the $\pi^{+} \pi^{-}$system in $X \rightarrow \pi^{+} \pi^{-} J / \psi$ decays originates from $\rho^{0} \rightarrow \pi^{+} \pi^{-}$decay, which is consistent with the $J^{P C}=1^{++}$assignment reported by LHCb. The decay $X \rightarrow \rho J / \psi$ implies that either the $X(3872)$ has isospin $I=1$ or 0 : if it has isospin zero, its decay to $\rho J / \psi$ is isospin violating; if it has $I=1$, it should have charged partners. Searches for narrow, charged partner states decaying to $\rho^{ \pm} J / \psi$ by BaBar [106] and Belle [102] set branching ratio limits well below expectations for isospin conservation. However, these limits should probably be viewed with some caution because if charged partners of the neutral $X(3872)$ exist and have masses that are even just a few $\mathrm{MeV}$ higher, their decays to $D \bar{D}^{*}$ final states would probably be significantly stronger than those for their neutral partner; these higher rates could suppress the $\rho^{ \pm} J / \psi$ decay branching fraction below isospin expectations and produce a significantly broader width. However, other evidence against $I=1$ comes from observations by Belle [107] and BaBar [72, 73] of $X(3872) \rightarrow \omega J / \psi$ decays with a branching fraction that is nearly equal to that for $\rho J / \psi$ decays; the PDG average is $\mathcal{B}(X(3872) \rightarrow$ $\omega J / \psi) / \mathcal{B}\left(X(3872) \rightarrow \pi^{+} \pi^{-} J / \psi\right)=0.8 \pm 0.3$ [13]. Since $M_{X(3872)}-m_{J / \psi} \simeq 775 \mathrm{MeV}$, and $\sim 7 \mathrm{MeV}$ below $m_{\omega}$, the decay $X(3872) \rightarrow \omega J / \psi$ can only proceed via the low energy tail of the $\omega$ peak and is, therefore, kinematically suppressed. I estimate a suppression factor of $\sim 1 / 120^{9)}$. The fact that this strongly suppressed $I=0$ final state is accessed at nearly the same rate as the $I=1, \rho J / \psi$ final state - which is also kinematically suppressed, but only by a factor that I estimate to be $\sim 1 / 5$ - suggests that the $X(3872)$ is (mostly) an isospin singlet and that the $\rho J / \psi$ decay mode violates isospin symmetry.

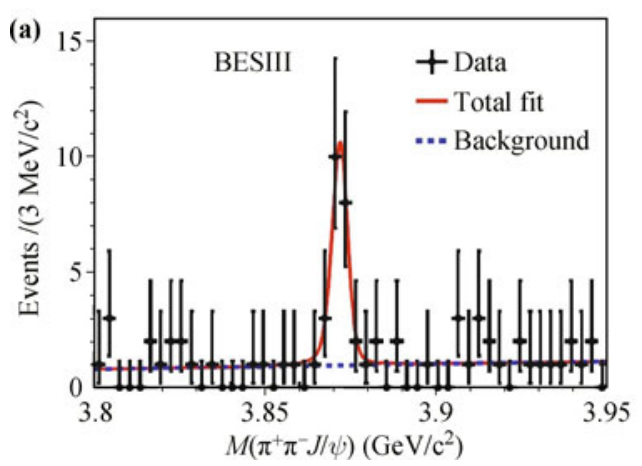

The $X(3872)$ has also been seen to decay to $D^{0} \bar{D}^{* 0}$ [108-110]. Since its $J^{P C}=1^{++}$, the $X(3872)$ couples to a $D \bar{D}^{*}$ pair in an $S$ - and/or $D$-wave. The $D^{0} \bar{D}^{* 0}$ system is right at threshold and, so, the $S$-wave should be dominant, in which case some very general universal theorems apply [111-113]. One consequence of these theorems is that, independently of its dynamical origin, the $X(3872)$ should exist for some fraction of the time as a $D \bar{D}^{*}$ molecule-like state (either bound or virtual) with size determined by its scattering length $a_{00}$, which in turn depends upon the mass difference $\delta m_{00}=m_{X(3872)}-m_{D^{0}}-m_{D^{* 0}}(=-0.09 \pm 0.28 \mathrm{MeV})$ as $a_{00}=\hbar / \sqrt{\mu \delta m_{00}}$, where $\mu$ is the $D \bar{D}^{*}$ reduced mass. This, taken with the experimentally allowed range of $\delta m_{00}$ values, suggests that for the $D^{0} \bar{D}^{0 *}$ configuration, the mean $D^{0}-\bar{D}^{* 0}$ separation is huge, of order $a_{00} \sim 10$ $\mathrm{fm}$, and much larger than the extent of the $D^{+} D^{*-}$ configuration, for which $\delta m_{+-}=m_{X(3872)}-m_{D^{+}}-m_{D^{*-}} \simeq$ $8 \mathrm{MeV}$ and $a_{+-} \simeq 2 \mathrm{fm}$. The very different properties of the $D^{0} \bar{D}^{* 0}$ and $D^{+} D^{*-}$ configurations imply that the $X(3872)$ isospin is not very well defined [114].

The BESIII experiment recently reported the observation of the process $e^{+} e^{-} \rightarrow \gamma X(3872)$ at cms energies in the region of the $Y(4260)$ peak [115]. The $X(3872)$ was detected via its $\pi^{+} \pi^{-} J / \psi$ decay channel; a $\pi^{+} \pi^{-} J / \psi$ invariant mass plot summed over the data at all of the energy points is shown in Fig. 18(a), where a $6.3 \sigma$ peak at the mass of the $X(3872)$ is evident. Figure 18(b) shows the energy dependence of the $X(3872)$ production rate where there is some indication that the observed signal is associated with the $Y(4260)$. Assuming that $Y(4260) \rightarrow$ $\gamma X(3872)$ decays are the source of this signal, and using the PDG lower limit $\mathcal{B}\left(X(3872) \rightarrow \pi^{+} \pi^{-} J / \psi\right)>0.026$

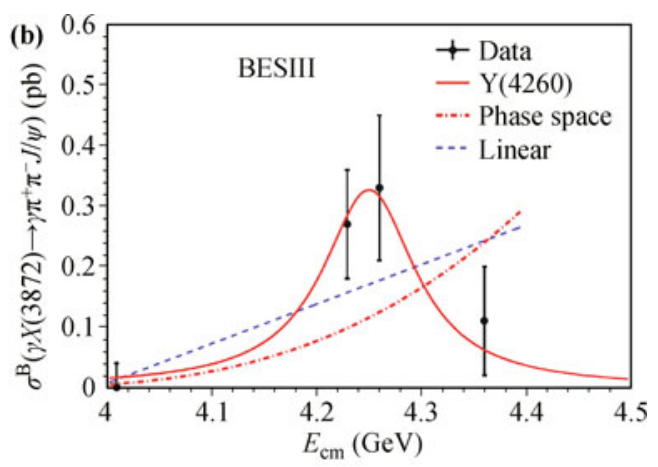

Fig. 18 (a) The data points show the BESIII experiment's $M\left(\pi^{+} \pi^{-} J / \psi\right)$ distribution for $e^{+} e^{-} \rightarrow \gamma \pi^{+} \pi^{-} J / \psi$ events at energies near the $Y(4260)$ resonance. The fitted peak has a mass and width of $M=3871.9 \pm 0.7 \mathrm{MeV}$ and $\Gamma=0.0_{-0.0}^{+1.7}$ $\mathrm{MeV}(<2.4 \mathrm{MeV})$, which are in good agreement with the PDG world average values for the $X(3872)$. (b) The energy dependence of the BESIII $\sigma^{B}\left(e^{+} e^{-} \rightarrow \gamma X(3872)\right) \times \mathcal{B}\left(X(3872) \rightarrow \pi^{+} \pi^{-} J / \psi\right)$, measurement where $\sigma^{B}$ denotes the Born cross section. The solid curve is the $Y(6260)$ line shape fitted to the data; the dashed curves show phase-space and linear production model expectations.

\footnotetext{
9) This estimate neglects the possible effects of $\rho-\omega$ interference.
} 
[13], we determine

$$
\frac{\mathcal{B}(Y(4260) \rightarrow \gamma X(3872))}{\mathcal{B}\left(Y(4260) \rightarrow \pi^{+} \pi^{-} J / \psi\right)}>0.05,
$$

which is substantial and suggestive of some commonality in the nature of the $Y(4260), X(3872)$ and $Z_{c}(3900)$.

Various interpretations for the $X(3872)$ have been proposed. The only viable candidate for a charmonium assignment is the $2^{3} P_{1}$ state, i.e., the $\chi_{c 1}^{\prime}$, which is the only charmonium level with the right quantum numbers and an expected mass that is anywhere near $3872 \mathrm{MeV}$. The measured mass is below the range of the theoretically expected values discussed in Ref. [76], where a computation using a non-relativistic potential model (NR) gives 3925 $\mathrm{MeV}$ while that based on a relativized potential model (GI) [36] gives $3953 \mathrm{MeV}$. Potential model calculations of masses of charmonium states that are above the opencharm thresholds at $2 m_{D}$ and $m_{D}+m_{D^{*}}$ do not explicitly take into account effects of virtual, but on-mass-shell $D \bar{D}$ and $D \bar{D}^{*}$ states and, so, it is not too surprising that both models miss the mass of the well measured multiplet partner $\chi_{c 2}^{\prime}$ by a substantial amount: NR gives 3972 $\mathrm{MeV}$ and GI gives $3979 \mathrm{MeV}$, while the PDG average of measured values is $\sim 50 \mathrm{MeV}$ lower at $3927.2 \pm 2.7 \mathrm{MeV}$. In the absence of any computation that incorporates the measured $\chi_{c 2}^{\prime}$ as input, I estimate a range of expected $\chi_{c 1}^{\prime}$ mass values pegged to the $m_{\chi_{c 2}^{\prime}}$ measurement to be $3880 \mathrm{MeV} \leqslant M\left(\chi_{c 1}^{\prime}\right) \leqslant 3901 \mathrm{MeV}$ by subtracting the NR and GI calculated $\delta m_{2-1}=m\left(\chi_{c 2}^{\prime}\right)-m\left(\chi_{c 1}^{\prime}\right)$ splittings, namely $\delta m_{2-1}(\mathrm{NR})=47 \mathrm{MeV}$ and $\delta m_{2-1}(\mathrm{GI})=26$ $\mathrm{MeV}$, from the $\chi_{c 2}^{\prime}$ measurement. The $X(3872)$ mass is below this range, but not by much. The biggest problem with the $\chi_{c 1}^{\prime}$ charmonium assignment for the $X(3872)$ is the strength of the $\rho J / \psi$ discovery decay channel, which, for charmonium, is isospin- and OZI-violating and expected to be strongly suppressed.

The close match between the $X(3872)$ mass and $m_{D^{0}}+$ $m_{D^{* 0}}$ - they are equal to within 1 part in $10^{4}$ - suggests that there is some close relation between the $X(3872)$ and the $D^{0} \bar{D}^{* 0}$ system. In 1994, Törnqvist proposed the existence of deuteron-like $D \bar{D}^{*}$ isoscalar states bound by pion exchange with $J^{P C}=0^{-+}$and $1^{++}$and mass near $3870 \mathrm{MeV}$ that he called "deusons" [116]. In August 2003, shortly after the $X(3872)$ discovery was announced by Belle at the Fermilab Lepton-Photon 2003 meeting, he identified it with the $1^{++} D \bar{D}^{*}$ deuson predicted in his 1994 paper. Because of its proximity to the $D^{0} \bar{D}^{* 0}$ threshold, the deuson wave function is predominantly $D^{0} \bar{D}^{* 0}$, and not a very pure isospin eigenstate as discussed above [114], and this can account for the strong isospin violation. There has been a considerable number of subsequent reports that describe the
$X(3872)$ as a molecule [118-129], an idea that predates even Törnqvist's 1994 paper [130-133]. However, the CDF group reported that only $(16.1 \pm 5.3) \%$ of their $X(3872)$ signal are produced via $B$ meson decays; most of their observed signal is produced promptly in high energy $p \bar{p}$ interactions [134]. In addition, the D0 experiment showed that the many of the characteristics of $X(3872)$ production in these high energy $p \bar{p}$ collisions, such as the rapidity- and $p_{T}$-dependence of the inclusive production cross section, are very similar to those for the $\psi^{\prime}$. The LHCb [99] and CMS [135] experiments report similar characteristics for inclusive $X(3872)$ production in $\sqrt{s}=7 \mathrm{TeV}$ proton-proton collisions. If the $X(3872)$ is a large and fragile molecule with a miniscule binding energy, why would its production characteristics in high energy $p p$ and $p \bar{p}$ collisions match those of the nearly point-like and tightly bound $\psi^{\prime} c \bar{c}$ state? This issue is discussed in detail in Ref. [136], where the authors use the powerful software tools that successfully model particle production in high energy hadron colliders to show that the prompt production cross section for a loosely bound $D^{0} \bar{D}^{* 0}$ molecule should be about two-orders-ofmagnitude smaller than the production rates measured by CDF.

Both Belle [138] and BaBar [137] reported similar strengths for $X(3872)$ decay to $\gamma J / \psi$ final states; the average of their measured branching fractions correspond to a $X(3872) \rightarrow \gamma J / \psi$ partial width that is less than that for $X(3872) \rightarrow \pi^{+} \pi^{-} J / \psi$ by a factor of $0.24 \pm 0.05$. This is in good agreement with a calculation based on a molecular picture by Aceti, Molina and Oset [139], where they find this ratio to be 0.18 . In addition to $D^{0} \bar{D}^{* 0}$, this calculation includes $D^{+} D^{*-}$ and $D_{s}^{+} D_{s}^{*-}$ components in the $X(3872)$ wave function. Interestingly, the authors find that the $\Gamma(\gamma J / \psi) / \Gamma\left(\pi^{+} \pi^{-} \psi\right)$ partial width ratio is very sensitive to these extra terms: if only the $D^{0} \bar{D}^{* 0}$ term is considered, the ratio becomes very much smaller, of order $\sim 10^{-4}$. Since many calculations based on a molecule picture ignore the $D^{+} D^{*-}$ and (especially) the $D_{s}^{+} D_{s}^{*-}$ terms, their reliability may be questionable.

Swanson pointed out that the relative partial widths for the $X(3872) \rightarrow \gamma \psi^{\prime}$ and $X(3872) \rightarrow \gamma J / \psi$ decay channels would be a powerful diagnostic for the $X(3872)$ [140]. For a pure $\chi_{c 1}^{\prime}$ charmonium state, he estimates that this ratio would be in the range $0.7-6.8$, while for pure $D \bar{D}^{*}$ molecular state the expectations are distinctly smaller, at $\sim 3 \times 10^{-3}$. Until recently, the situation with $X(3872) \rightarrow \gamma \psi^{\prime}$ was not very clear. BaBar reported $3.5 \sigma$ evidence for this mode at a rate that is $3.4 \pm 1.4$ times that for $\gamma J / \psi[137]$; Belle reported no signal and a 90\% CL upper limit on this ratio of 2.1 [138]. This year LHCb study of this mode measured a 
$4.4 \sigma$ signal for $X(3872) \rightarrow \gamma \psi^{\prime}$, and a ratio relative to an $X(38972) \rightarrow \gamma J / \psi$ signal in the same data of $2.5 \pm 0.7$ [141], much higher than expectations for a pure $D \bar{D}^{*}$ molecular state.

Maiani and collaborators advocate a QCD-type tetraquark structure (i.e., a $c q$ diquark and a $\bar{c} \bar{q}$ diantiquark, where $q=d$ or $u$ ) for the $X(3872)$ [142]. In their first paper on the subject, they predicted that the $X(3872)$ would be one of two neutral states that differ in mass by $8 \pm 3 \mathrm{MeV}$, a value that is related to the $u$ - and $d$-quark mass difference; one of the states was predicted to be produced in $B^{+} \rightarrow K^{+} X_{1}(3872)$ and the other in $B^{0} \rightarrow K^{0} X_{2}(3872)$ decays. They also predicted the existence of charged partner states, $X^{ \pm}$, nearby in mass. BaBar [106] and Belle [102] studied these possibilities and found no evidence for different $X(3872)$ mass differences in $B^{+}$and $B^{0}$ decays and put limits on $\mathcal{B}\left(B \rightarrow K X^{ \pm}\right) \times \mathcal{B}\left(X^{ \pm} \rightarrow \pi^{ \pm} \pi^{0} J / \psi\right.$ that are well below isospin-based predictions. However, the recent discovery of the $Z_{c}(3900)^{ \pm, 0}$ triplet has supplied the "missing" particles, albeit with different $G$-parity and at somewhat higher masses. This revived interest in QCD-tetraquark ideas [143]. Among other things, this model has a natural explanation for the $Z(4430)$ [144] and, since QCDtetraquarks are tightly bound, compact objects, their prompt production in high hadron collisons with properties can be expected to be similar to that for the $\psi^{\prime}$. On the other hand, Ref. [143] proposed a second $Z_{c}$ state at about $100 \mathrm{MeV}$ below the $Z_{c}(3900)$ mass and contrasted this prediction with a molecule expectation for a second $Z_{c}$ existence of another $\pi^{+} J / \psi$ near the $D^{*} \bar{D}^{*}$ threshold. The subsequent observation by BESIII [51] of the higher mass $Z_{c}(4020)$ near the $2 m_{D^{*}}$ threshold clearly favors the molecule scheme.

QCD-hybrid mesons are color-octet quark-antiquark combinations with an excited gluon degree of freedom [145]. While there is considerable literature that identifies the $Y(4260)$ as a charmonium hybrid, i.e., a $c \bar{c}$-gluon structure [46, 47], there have been no suggestions that this idea may apply to the $X(3872)$ as well. This may be partly due to the fact that LQCD calculations find the lowest $1^{++}$charmonium hybrid mass to be near 4400 $\mathrm{MeV}$ and far above that of the $X(3872)$ [146].

Hadrocharmonium is a model in which a $Q \bar{Q}$ pair forms a tightly bound system embedded in a light mesonic cloud that it interacts with via QCD analogs of Van der Waals forces [130, 147]. The $Q \bar{Q}$ core states have wave functions that are closely related to conventional Quarkonium states. This would explain, for example, the dominance of $Z(4430) \rightarrow \pi \psi^{\prime}$ over $\pi J / \psi$ de- cays. The proponents of this model have not made any $X(3872)$-specific predictions.

The defects in all of the above-mentioned pictures for the $X(3872)$ have inspired models that incorporate combinations of the different ideas. For example, a charmonium-molecular hybrid ${ }^{10)}$ model by Takizawa and Takeuchi finds a specific $X(3872)$ wave function [148]:

$$
\begin{aligned}
|X(3872)\rangle= & 0.237|c \bar{c}\rangle-0.944\left|D^{0} \bar{D}^{* 0}\right\rangle \\
& -0.228\left|D^{+} D^{*-}\right\rangle,
\end{aligned}
$$

which translates into about $6 \% c \bar{c}, 69 \%$ of isoscalar $D \bar{D}^{*}$ and $26 \%$ isovector $D \bar{D}^{*}$. Hadronic production is hypothesized to proceed via the $c \bar{c}$ core component and this could explain why $X(3872)$ production properties are similar to those of the $\psi^{\prime}$. This calculation uses a high $\chi_{c 1}^{\prime}$ input mass, namely $3950 \mathrm{MeV}$, which may result in an underestimate of the $c \bar{c}$ component, but does not negate the basic idea. Note that similar numerical results are found in Ref. [113].

There are two related states that are orthogonal to the one given in Eq. (2). Reference [148] discusses one that is mostly $c \bar{c}$ and probably should be considered to be the $\chi_{c 1}^{\prime}$ charmonium state. This is pushed up in mass to well above both $D \bar{D}^{*}$ thresholds and becomes wide and not easily identifiable. Presumably the other state would be dominantly isovector $D \bar{D}^{*}$, and this might be the $Z_{c}(3900)^{0}$, the neutral $I_{z}=0$ member of the $Z_{c}(3900)^{ \pm, 0}$ isospin triplet. If so, the residual $c \bar{c}$ core and isoscalar $D \bar{D}^{*}$ components may result in a measureable isospin violating differences between $Z_{c}(3900)^{0} \rightarrow D^{0} \bar{D}^{* 0}$ and $Z_{c}(3900)^{0} \rightarrow D^{+} D^{*-}$ decays.

The $X(3872)$ has a long interesting story that we cannot do justice to in this brief report and, instead, refer the readers to a recent review [149].

\subsubsection{Bottomoniumlike mesons}

Figure 19 indicates the recent status of the $b \bar{b}$ bottomonium and bottomoniumlike mesons. Here the beige boxes indicate the well established bottomonium mesons, the green boxes show those that were recently established, and the red and purple boxes indicate anomalous states that are discussed below.

The large $Y(4260) \rightarrow \pi^{+} \pi^{-} J / \psi$ signal discovered in the charmonium mass region by BaBar motivated a Belle search for similar behavior in the bottomonium system [150]. This uncovered anomalously large $\pi^{+} \pi^{-} \Upsilon(n S)$ $(n=1,2,3)$ production rates that peak around $\sqrt{s}=$ $10.89 \mathrm{GeV}$ as shown in the upper panel of Fig. 20(a)

10) This "hybrid" is not the same as the previously discussed QCD hybrid. 
[151]. This peak energy is about $2 \sigma$ higher than that of the peak in the $e^{+} e^{-} \rightarrow$ hadron cross section at $\sqrt{s} \simeq 10.87 \mathrm{GeV}$ that is usually associated with the conventional $\Upsilon(5 S)$ bottomonium meson (and shown in the lower panel of Fig. 20(b). If the peak in the $\pi^{+} \pi^{-} \Upsilon(n S)$ cross section is attributed to $\Upsilon(5 S)$ decays, it implies $\Upsilon(5 S) \rightarrow \pi^{+} \pi^{-} \Upsilon(n S)(n=1,2,3)$ partial widths that are hundreds of times larger than theoretical predictions [152], and the corresponding measured rates for the $\Upsilon(4 S)[13]$. This suggests the presence of a new, non- $b \bar{b} b$ quark-sector equivalent of the $Y(4260)$ with mass around $10.89 \mathrm{GeV}$ [153] and referred to in the following as the $\Upsilon(10890)$.

The large cross sections for $e^{+} e^{-} \rightarrow \pi^{+} \pi^{-} \Upsilon(n S)$ $(n=1,2,3)$ near the $\Upsilon(5 S)$ motivated Belle to take a large sample of data at this energy in order to inves- tigate the source of this anomaly. By the end of Belle operations, a $121.4 \mathrm{fb}^{-1}$ data sample had been accumulated near the peak of the $\Upsilon(5 S)(\sqrt{s}=10.87 \mathrm{GeV})$ and data taken in energy scans around the peak accounted for an additional sample of $12 \mathrm{fb}^{-1}$. One of the first studies done with this $\Upsilon(5 S)$ data sample was an investigation of the inclusive missing-mass spectrum recoiling from $\pi^{+} \pi^{-}$ pairs.

The upper right panel of Fig. 20 shows the distribution of masses recoiling against all of the $\pi^{+} \pi^{-}$pairs in events collected near the peak of the $\Upsilon(5 S)$ resonance [154]. The combinatoric background is huge - there are typically $10^{6}$ entries in each $1 \mathrm{MeV}$ bin - and the statistical errors are tiny $(\sim 0.1 \%)$. The data were fit piece-wise with sixth-order polynomials, and the residuals from the fits are shown in the lower panel of Fig. 20(b), where, in

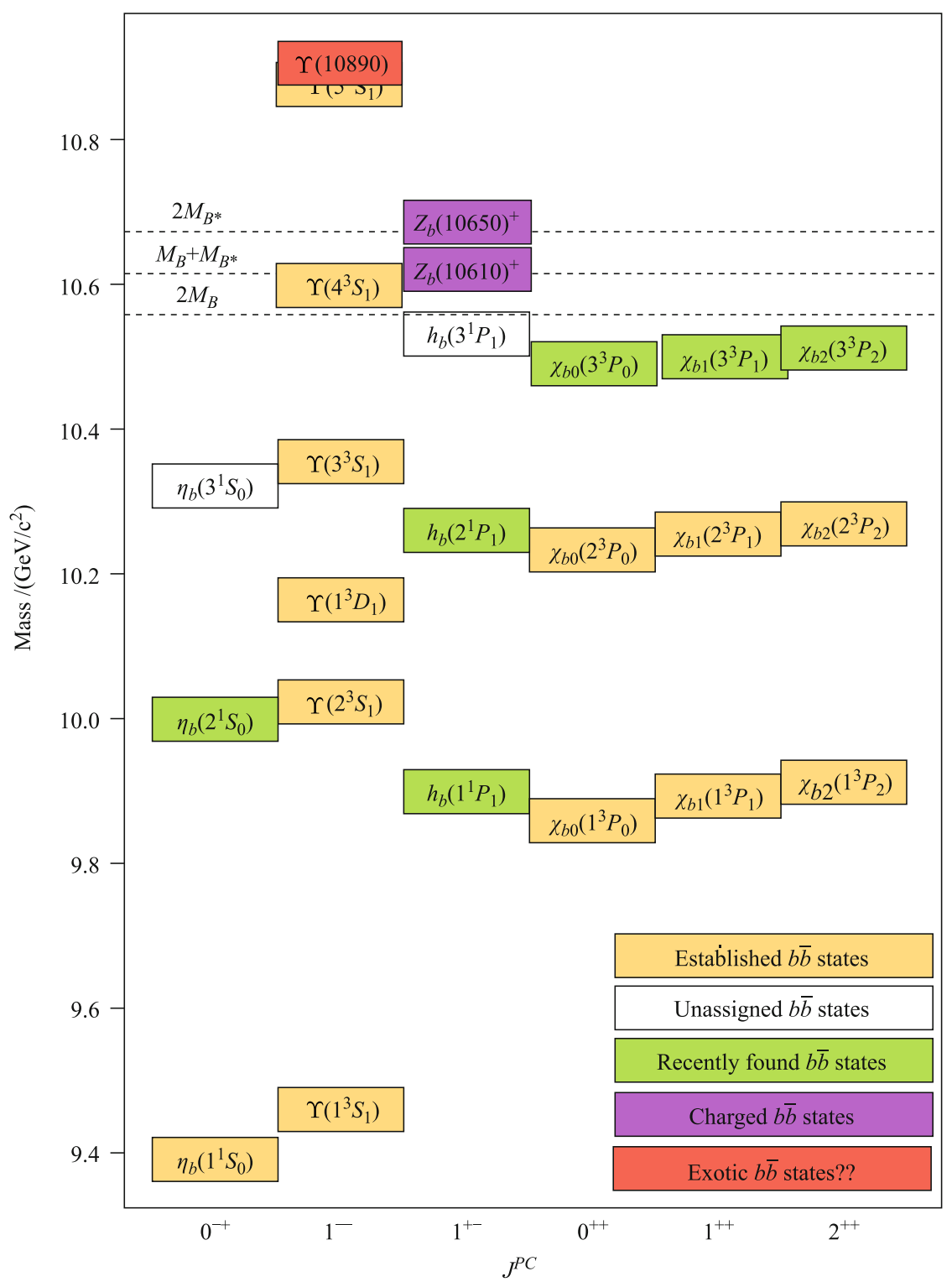

Fig. 19 The spectrum of bottomonium and bottomoniumlike mesons. 

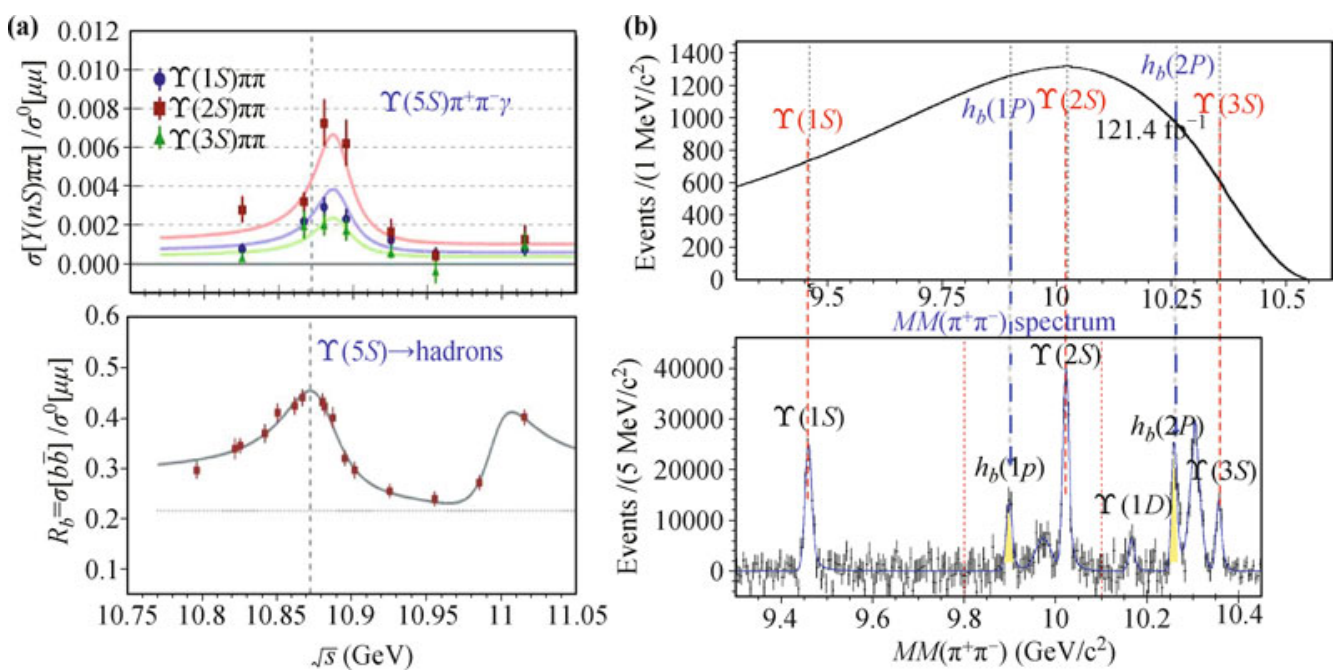

Fig. 20 (a) Cross sections for $e^{+} e^{-} \rightarrow \pi^{+} \pi^{-} \Upsilon(n S)(n=1,2,3)$ (upper) and $e^{+} e^{-} \rightarrow$ hadrons (lower) in the vicinity of the $\Upsilon(5 S)$ resonance (from Ref. [151]). (b) Distribution of masses recoiling against $\pi^{+} \pi^{-}$pairs at cms energies near 10.87 $\mathrm{GeV}$ (upper) and residuals from piecewise fits to the data with smooth polynomials (lower) (from Ref. [154]). The $h_{b}(1 P)$ and $h_{b}(2 P)$ peaks, shaded in yellow, are first observations.

addition to peaks at the $\Upsilon(1 S), \Upsilon(2 S), \Upsilon(3 S)$ masses and some expected reflections, there are unambiguous signals for the $h_{b}(1 P)$ and $h_{b}(2 P)$, the $1^{1} P_{1}$ and $2^{1} P_{1}$ bottomonium states. This was the first observation of these two elusive levels [154]. One puzzle is that the $\pi^{+} \pi^{-} h_{b}(m S),(m=1,2)$ final states are produced at rates that are nearly the same as those for $\pi^{+} \pi^{-} \Upsilon(n S)$ $(n=1,2,3)$, even though the $\pi^{+} \pi^{-} h_{b}$ transition requires a heavy-quark spin flip that should result in a strong suppression. This motivated a more detailed investigation of these channels.

Figure 21(a) shows the $\pi^{+} \pi^{-} h_{b}$ yields versus the maximum $h_{b} \pi^{ \pm}$invariant mass for $h_{b}=h_{b}(1 P)$ (upper) and $h_{b}=h_{b}(2 P)$ (lower), where it can be seen that all of the $\pi^{+} \pi^{-} h_{b}$ events are concentrated in two $M_{\max }\left(h_{b} \pi\right)$ peaks near $10610 \mathrm{MeV}$ and and $10650 \mathrm{MeV}$ [155]. Studies of fully reconstructed $\pi^{+} \pi^{-} \Upsilon(n S),(n=1,2,3)$ $\Upsilon(n S) \rightarrow \ell^{+} \ell^{-}$events in the same data sample found peaks at the same masses in the $M_{\max }(\Upsilon(n S) \pi)$ distributions for all three $\Upsilon(n S)$ states; these are shown in the three center panels of Fig. 21. Here the fractions of $\pi^{+} \pi^{-} \Upsilon(n S)$ events in the two peaks are substantial $\sim 6 \%$ for the $\Upsilon(1 S), \sim 22 \%$ for the $\Upsilon(2 S)$ and $\sim 43 \%$ for the $\Upsilon(3 S)$ - but, unlike the case for the $\pi^{+} \pi^{-} h_{b}(m P)$ channels, they do not account for all of the $\pi^{+} \pi^{-} \Upsilon(n S)$ event yield [156]. Fitted values of the peak masses and widths in all five channels are consistent with each other; the weighted average mass and width values are

$$
\begin{aligned}
Z_{1}(10610): & M_{1}=10607.2 \pm 2.0 \mathrm{MeV} \\
& \Gamma_{1}=18.4 \pm 2.4 \mathrm{MeV} \\
Z_{2}(10650): & M_{2}=10552.2 \pm 1.5 \mathrm{MeV} \\
& \Gamma_{2}=11.5 \pm 2.2 \mathrm{MeV} .
\end{aligned}
$$

A study of $\pi^{0} \pi^{0} \Upsilon(n S),(n=1,2,3)$ found a $6.5 \sigma$ signal for the neutral $Z_{b}(10610)^{0}$ isospin partner state with a mass $M_{Z_{b}(10610)^{0}}=10609 \pm 6 \mathrm{MeV}$ and a production rate that is consistent with isospin-based expectations [157].

The lower mass state is just $2.6 \pm 2.2 \mathrm{MeV}$ above the $m_{B}+m_{B^{*}}$ mass threshold and the higher mass state is only $2.0 \pm 1.6 \mathrm{MeV}$ above $2 m_{B^{*}}$. Dalitz-plot analyses of the $\pi^{+} \pi^{-} \Upsilon(n S)$ final states establish $J^{P}=1^{+}$quantum number assignments for both states $[158,159]$. The close proximity of the $B \bar{B}^{*}$ and $B^{*} \bar{B}^{*}$ thresholds and the $J^{P}=1^{+}$quantum number assignment is suggestive of virtual $S$-wave molecule-like states.

The $B^{(*)} \bar{B}^{*}$ molecule picture is supported by a Belle study of $e^{+} e^{-} \rightarrow B^{(*)} \bar{B}^{*} \pi$ final states in the same data sample [160], where $B \bar{B}^{*}$ and $B^{*} \bar{B}^{*}$ invariant mass peaks are seen at the $Z_{b}(10610)$ and $Z_{b}(10650)$ mass values, respectively, as shown in the right panels of Fig. 21. From these data, preliminary values of the branching fractions $\mathcal{B}\left(Z_{b}(10610) \rightarrow B^{+} \bar{B}^{* 0}+\bar{B}^{0} B^{*+}+\right.$ c.c. $)=(86.0 \pm 3.6) \%$ and $\mathcal{B}\left(Z_{b}(10610) \rightarrow B^{*+} \bar{B}^{* 0}+\right.$ c.c. $)=(73.4 \pm 7.0) \%$ are inferred. The $B^{(*)} \bar{B}^{*}$ "fall apart" modes are stronger than the sum total of the $\pi^{+} \Upsilon(n S)$ and $\pi^{+} h(m P)$ modes, but only by factors of $\sim 6$ for the $Z_{b}(10610)$ and $\sim 3$ for the $Z_{b}(10650)$. The measured branching fraction for $Z_{b}(10610) \rightarrow B^{*} \bar{B}^{*}$ is consistent with zero. This pattern, where $B \bar{B}^{*}$ decays dominate for the $Z_{b}(10610)$ and $B^{*} \bar{B}^{*}$ decays are dominant for the $Z_{b}(10650)$ are consistent with expectations for molecule-like structures.

\subsection{Comments}

Table 1 provides a tabulation of recently discovered 


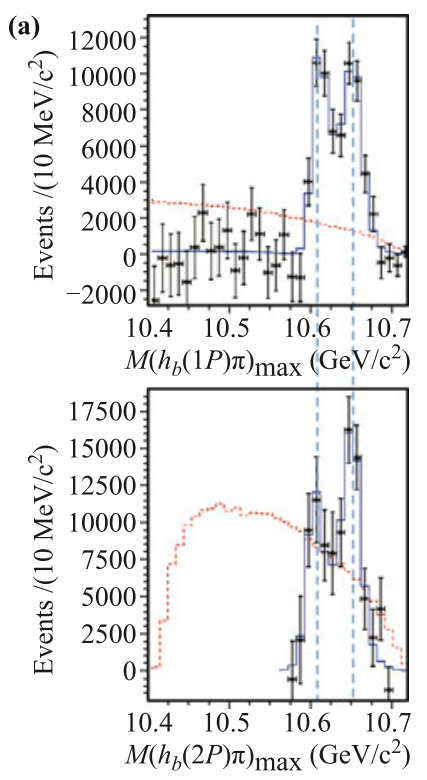

(b)
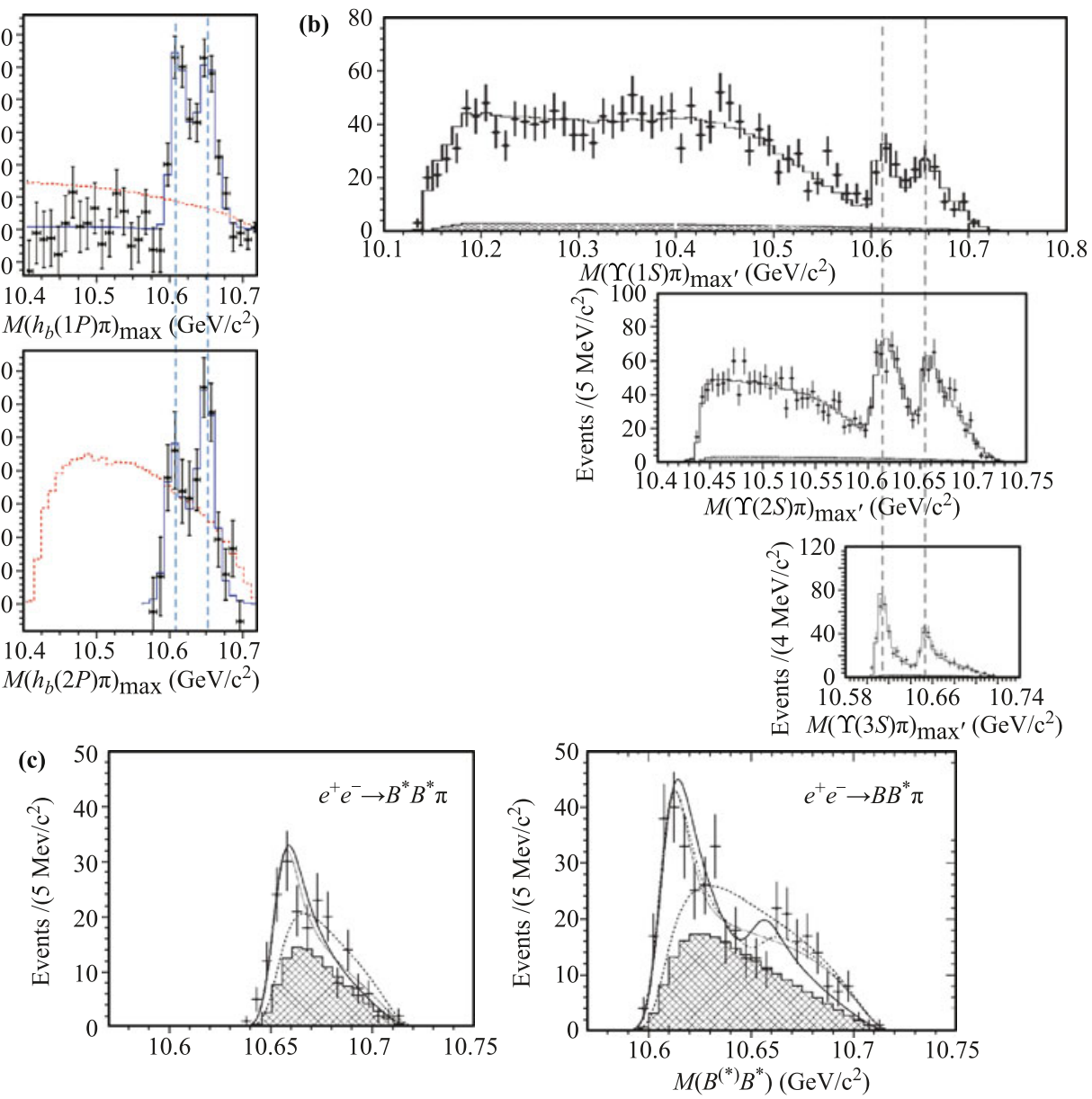

Fig. 21 (a) Invariant mass distributions for $h_{b}(1 P) \pi^{+}$(upper) and $h_{b}(2 P) \pi^{+}$(lower) from $e^{+} e^{-} \rightarrow \pi^{+} \pi^{-} h_{b}(n P)$ events. (b) Invariant mass distributions for $\Upsilon(1 S) \pi^{+}$(upper), $\Upsilon(2 S) \pi^{+}$(center) and $\Upsilon(3 S) \pi^{+}$(lower) in $e^{+} e^{-} \rightarrow \pi^{+} \pi^{-} \Upsilon(n S)$ events. The figures are from Ref. [155], and scaled to make the make the horizontal scales (almost) match. (c) The $B^{*} \bar{B}^{*}$ (upper) and $B \bar{B}^{*}$ (lower) invariant mass distributions for $e^{+} e^{-} \rightarrow B^{(*)} \bar{B}^{*} \pi$ events near $\sqrt{s}=10.86 \mathrm{GeV}$ (from Ref. [160]).

mesons (and candidate mesons), together with observed production \& decay channels, averages of mass and width measurements and the $J^{P C}$ values when they are known. For simplicity, I assume the $Z_{c}(3900) \rightarrow \pi^{+} J / \psi$ and $Z_{c}(3885) \rightarrow\left(D \bar{D}^{*}\right)^{+}$are the same state and average the measured mass and width values, the $Z_{c}(4020) \rightarrow \pi^{+} h_{c}$ and $Z_{c}(4025) \rightarrow\left(D^{*} \bar{D}^{*}\right)^{+}$measurements are treated in the same way.

\subsubsection{Molecules?}

The recent BESIII findings, taken together with previous experimental results, establishes a concentration of charmoniumlike states crowding the $D \bar{D}^{*}$ and $D^{*} \bar{D}^{*}$ mass threshold regions and bottomoniumlike isospin triplets near the $B \bar{B}^{*}$ and $B^{*} \bar{B}^{*}$ thresholds, which are suggestive of molecule-like structures [118-129].

If we assume that the $Z_{c}(3900)$ and $Z_{c}(3885)$ are the same state, it corresponds to an isospin triplet with a pole mass that is about $\sim 15 \mathrm{MeV}$ above the $D^{0} D^{*-}$ threshold with $J^{P}=1^{+}$and a significant coupling to $D \bar{D}^{*}$. This is consistent with a virtual $S$-wave $D \bar{D}^{*}$ molecule of the form $\left(D \bar{D}^{*}-D^{*} \bar{D}\right) / \sqrt{2}$. The $X(3872)$ also has $J^{P}=1^{+}$and couples to $D \bar{D}^{*}$ with mass right at the $D^{0} \bar{D}^{* 0}$ threshold and seems to be an isospin singlet [102]. This suggests an $S$-wave $D \bar{D}^{*}$ molecule of the form $\left(D \bar{D}^{*}+D^{*} \bar{D}\right) / \sqrt{2}$. (Note that although they both have $J^{P}=1^{+}$, the $X(3872)$ has even $C$ parity, while the $I_{z}=0$ member of the $Z_{c}(3900)$ triplet must have odd $C$ parity.)

The $Z_{c}(4020) / Z_{c}(4025)$ observations (assuming they are the same state) establish the existence of another isospin triplet just above the $D^{*} \bar{D}^{*}$ threshold. If this is the charmed-sector equivalent of the $Z_{b}(10650)$, it also has $J^{P}=1^{+}$and could be considered as a virtual $D^{*} \bar{D}^{*}$ molecule for which the $I_{z}=0$ component must have odd $C$ parity. This suggests that there might be an $X(3872)$ like, even $C$ parity isospin singlet $D^{*} \bar{D}^{*}$ nearby, and labeled as $X_{c 2}$ in the lower part of the level diagram shown in the left panel of Fig. 22. 
Table 1 New $c \bar{c}$ and $b \bar{b}$ mesons above open-flavor threshold. The masses $M$ and widths $\Gamma$ are weighted averages of measurements with uncertainties added in quadrature. For the $X(3872)$, only $\pi^{+} \pi^{-} J / \psi$ decays are used in the mass average. Ellipses (...) indicate an inclusive reaction. In the $J^{P C}$ column, question marks indicate my educated guess or no information. For charged states, $C$ is that of the neutral isospin partner. " $\Upsilon(5 S)^{\prime \prime}$ is in quotes to reflect the suspicion that the anomalous $\pi^{+} \pi^{-} \Upsilon(n S)$ events originate not from the $\Upsilon(5 S)$, but from another $1^{--}$meson with a nearby mass, i.e., the $\Upsilon(10890)$. This Table is a modification of one from Ref. [161] via Ref. [29, 30].

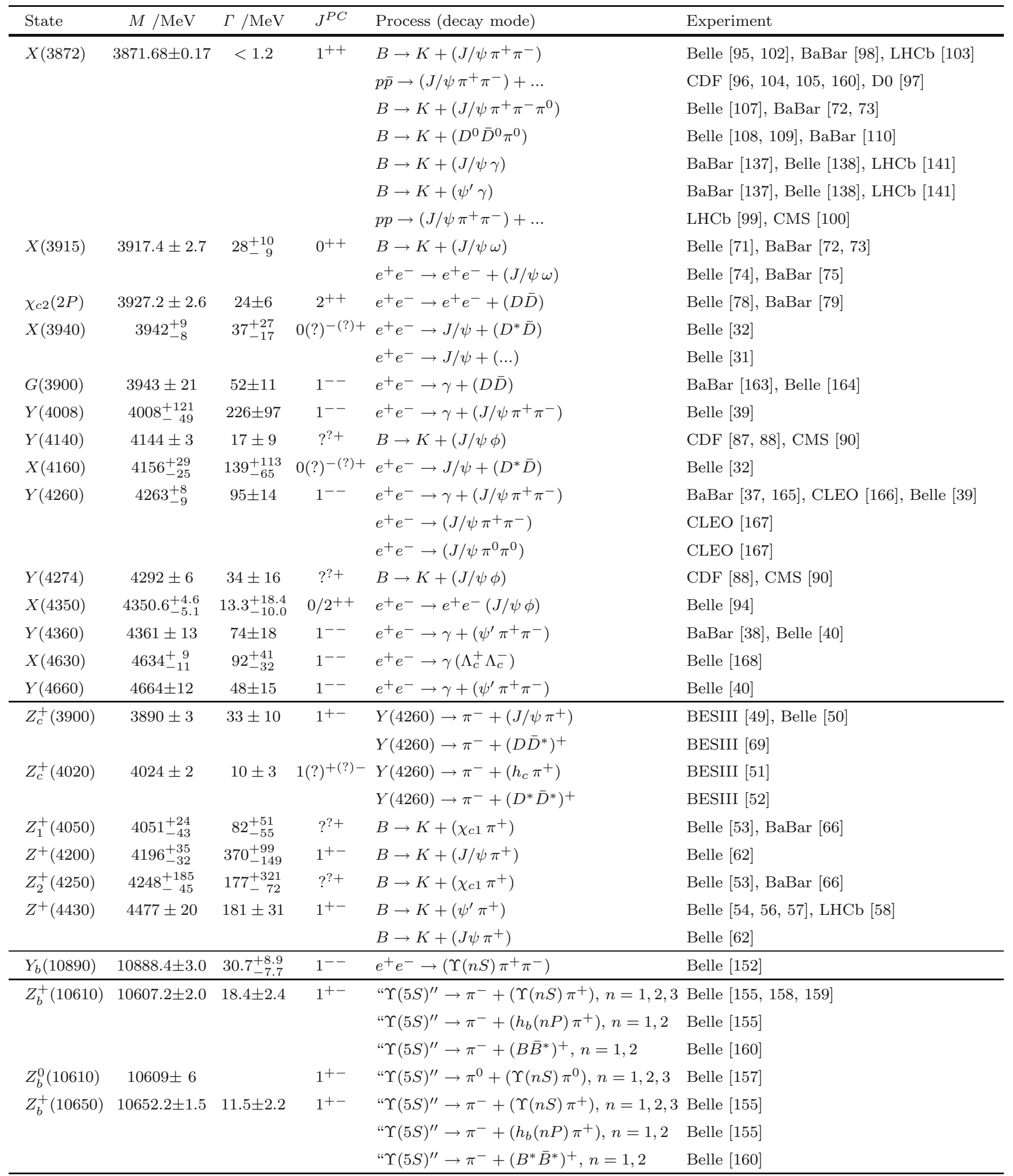


(a)

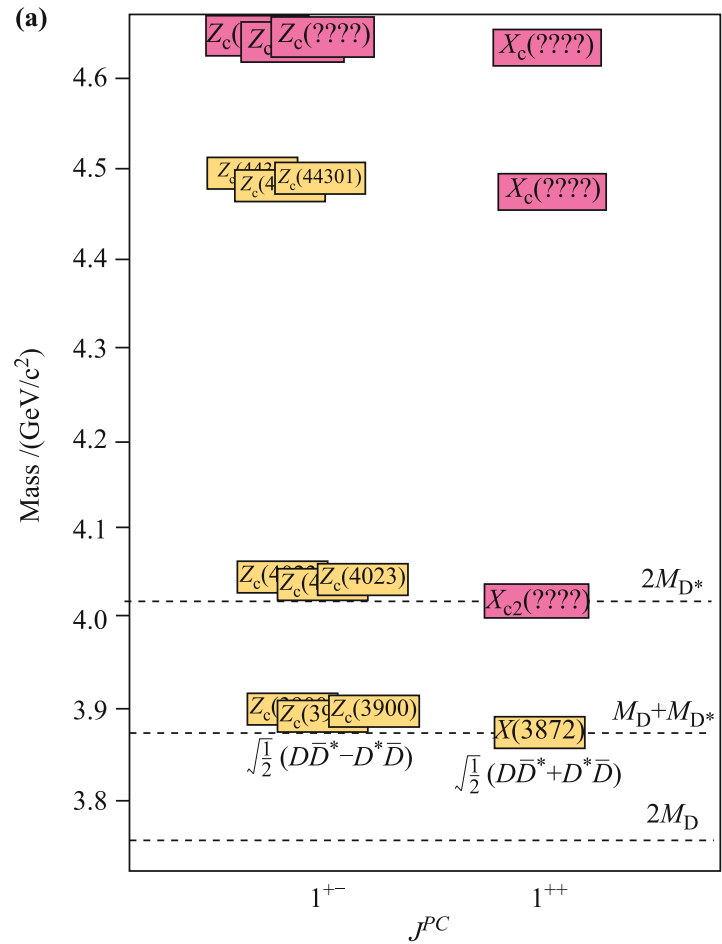

(b)

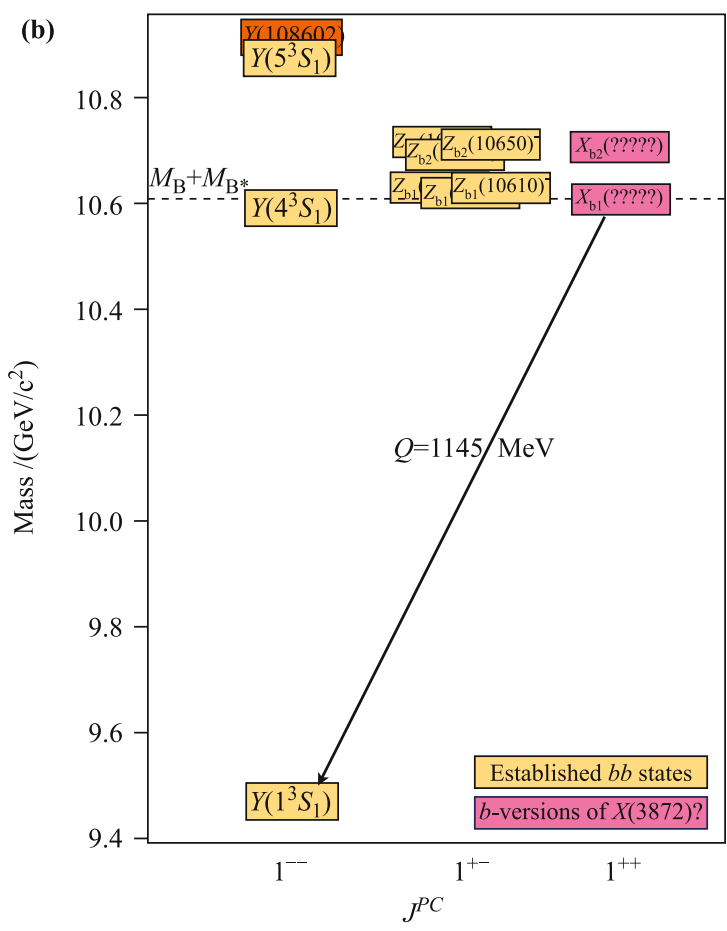

Fig. 22 (a) Level diagram for the $X(3872)$, the recently discovered $Z_{c}(3900) \& Z_{c}(4020)$ isotopic triplets, and the $Z(4430)$ isospin triplet. The salmon-colored boxes indicate other states that are suggested by the molecule picture. (b) Level diagram for the recently discovered $Z_{b}$ states, a conjectured b-sector equivalent of the $X(3872)$ at the $m_{B}+m_{B^{*}}$ threshold and an additional isoscalar partner of the $Z_{b}(10650)$ at the $2 m_{B^{*}}$ theshold. The transition between a $m_{B}+m_{B^{*}}$ threshold state to the $\Upsilon(1 S)$ would have a $Q$-value of $\simeq 1145 \mathrm{MeV}$, well above the mass of the $\rho$ and $\omega$ mesons.

If the $X_{c 2}$ exists with a mass that is below the $D^{*} \bar{D}^{*}$ threshold and a relatively narrow width, it might have a significant branching fraction to $\omega J / \psi$ final states. If its mass is above $2 m_{D^{*}}$ and it is relatively narrow, it might be accessible in $B^{-} \rightarrow K^{-} D^{*} \bar{D}^{*}$ decays. BaBar has reported large branching fractions for $B^{-} \rightarrow K^{-} D^{0 *} \bar{D}^{* 0}$ $(1.1 \pm 0.1 \%)$ and $K^{-} D^{*+} D^{*-}(0.13 \pm 0.02 \%)$ but did not publish any invariant mass distributions [169].

The $Z_{c}$ mesons have a minimal four-quark structure of $c \bar{c} q_{i} \bar{q}_{j}\left(i=1,2, j=1,2 \& q_{1}=u, q_{2}=d\right)$. The locations of the $Z_{c}(3900)$ and the $Z_{c}(4020)$ near the $D \bar{D}^{*}$ and $D^{*} \bar{D}^{*}$ thresholds and the similarity of the $M_{Z_{c}(4020)}-M_{Z_{c}(3900)}=133.9 \pm 4.0 \mathrm{MeV}$ mass splitting with the $\left\langle m_{D^{*}}-m_{D}\right\rangle \simeq 141 \mathrm{MeV}$ mass difference indicates that configurations where $c \bar{q}_{j}$ form a $D^{(*)}$ and $\bar{c} q_{j}$ form a $D^{*}$ are important, as is the case for molecular pictures. The BaBar group has recently identified the $n_{r}=2$ excitations of the $D$ and $D^{*}$ with masses $M_{D(2 S)}=2540 \pm 8 \mathrm{MeV}$ and $M_{D^{*}(2 S)}=2609 \pm 4 \mathrm{MeV}$ [1708]. An $S$-wave $D \bar{D}^{*}(2 S)$ combination would have have $J^{P}=1^{+}$and a "threshold" mass around $4480 \mathrm{MeV}$, very close to the Belle-LHCb average $M_{Z(4430)}=4477 \pm$ $19 \mathrm{MeV}$.

The charged $Z_{1}(4050)^{+}$and $Z_{2}(4250)^{+}$states that decay to $\pi^{+} \chi_{c 1}$ are less easy to associate with a thresh- old. We do not know their $J^{P}$ - we do not even know if the two states have the same $J^{P}$ values - but if the $\pi^{+}-\chi_{c 1}$ system is in an $S$-wave, $J^{P}=1^{-}$. We do know that their neutral $I_{3}=0$ partner, which has not yet been seen, has to have even $C$ parity. If we assume $J^{P}=1^{-}$, a $D \bar{D}_{1}(2420)$ would give the right quantum numbers and a nearby mass threshold for the $Z_{2}(4250)$, where the $D_{1}$ is a $J^{P}=1^{+}, D^{*} \pi$ resonance with mass $M=2421.3 \pm 0.6 \mathrm{MeV}$ and width $\Gamma=27.1 \pm 2.7 \mathrm{MeV}$. One could imagine a situation where the $Y(4260)$ is the $C$ odd isosinglet and the $Z_{2}(4250)$ is the $C$ even isotriplet $D \bar{D}_{1}$ molecular states $1 / \sqrt{2}\left(D \bar{D}_{1} \pm D_{1} \bar{D}\right)$. However, the scenario where the $Y(4260)$ is a $D \bar{D}_{1}$ molecule [48] was examined by BESIII as part of their study of $Y(4260) \rightarrow$ $\pi D \bar{D}^{*}[69]$. Since the $Y(4260)$ mass is $\sim 30 \mathrm{MeV}$ below $m_{D}+m_{D_{1}} \simeq 4290 \mathrm{MeV}$, a prominant $D \bar{D}_{1}$ component of its wave function should show up as a distinct clustering of $\pi \bar{D}^{*}$ invariant masses near their upper kinematic limit. BESIII reports no evidence for such clustering and, thus, no indication of a $D \bar{D}_{1}$ component of the $Y(4260)$. On the other hand, there are no interesting nearby $D^{(*)} \bar{D}^{(*)}$ mass thresholds for the $Z_{1}(4020)$, especially for $J^{P}=1^{-}$.

In the $b$-quark-sector, the $Z_{b}$ states are isospin triplets with $J^{P}=1^{+}$near the $B B^{*}$ and $B^{*} \bar{B}^{*}$ threshold and suggestive of virtual $S$-wave $B \bar{B}^{*}$ and $B^{*} \bar{B}^{*}$ molecules. 
Here we might expect $C$-even equivalents of the $X(3872)$ and $X_{c 2}$ (labeled as $X_{b 1}$ and $X_{b 2}$ in the right panel of Fig. $22)$, but finding them may be difficult with existing data. The CMS group searched for a $b$-quark version of the $X(3872)$ in the inclusive $\pi^{+} \pi^{-} \Upsilon(1 S)$ invariant mass distribution produced in proton-proton collisions at $\sqrt{s}=8$ $\mathrm{TeV}$, but found no evidence for peaks other than those due to $\Upsilon(2 S)$ and $\Upsilon(3 S)$ to $\pi^{+} \pi^{-} \Upsilon(1 S)$ transitions [171]. However, if, as expected, the $b$-quark-sector equivalent of the $X(3872)$ has $J^{P C}=1^{++}$, zero isospin, and is near the $B \bar{B}^{*}$ mass threshold, the $\pi^{+} \pi^{-} \Upsilon(1 S)$ decay mode, for which the $\pi^{+} \pi^{-}$would have to originate from $\rho \rightarrow$ $\pi^{+} \pi^{-}$, would violate isospin and be suppressed relative to decays to the isospin-conserving $\omega \Upsilon(1 S)$ final state. This is not the case for the $X(3872)$ where the isospinallowed $\omega J / \psi$ decay mode is kinematically suppressed: i.e., $Q_{c} \simeq m_{D^{0}}+m_{D^{* 0}}-m_{J / \psi}=776 \mathrm{MeV}$, which is about one $\omega$ natural width below its peak mass $m_{\omega}=783 \mathrm{MeV}$. In the $b$-quark-sector, $m_{B}+m_{B^{*}}-m_{\Upsilon(1 S)}=1145 \mathrm{MeV}$, which is well above $m_{\omega}$. Thus, $\omega \Upsilon(1 S)$ final states are probably more relevant than $\pi^{+} \pi^{-} \Upsilon(1 S)$ for searches for $X(3872)$ counterparts of the $Z_{b}(10610)$ and $Z_{b}(10650)$. This would require studies of decay final states that contain a $\pi^{0}$, which may be difficult to do with existing LHC experiments, but could be done at BelleII [85].

\subsubsection{Tetraquarks?}

Maiani and collaborators note that the $M_{Z(4430)}-$ $M_{Z_{c}(3900)}=589 \pm 30 \mathrm{MeV}$ mass difference is the same as $m_{\psi^{\prime}}-m_{J / \psi}=589 \mathrm{MeV}$, with not such a large error. This, together with the preference for the $Z(4430)^{+}$ to decay to $\pi^{+} \psi^{\prime}$ rather than $\pi^{+} J / \psi$, suggests that in the $Z(4430)$, configurations where the $c$ and $\bar{c}$ form a $\psi^{\prime}$ are important. This is in line with QCD tetraquark expectations as discussed in Ref. [144].

In Ref. [143], which appeared shortly after the $Z_{c}(3900)$ discovery was announced, Maiani and collaborators proposed a test between the tetraquark and molecular model: their model predicted a second charged $Z_{c}$ state with a mass that is about $100 \mathrm{MeV}$ lower, while the molecular picture predicted a second state at higher mass, close to the $2 m_{D^{*}}$ threshold. The BESIII group's subsequent discovery of the $Z(4020)$ confirmed the molecular picture. However, the Ref. [143] prediction of a lower mass partner state was based on a particular model for diquark-diantiquark spin-spin interactions. Perhaps modifications that incorporate measured properties of the $Z_{c}(4020)$ can fix this. The same paper also predicted that the $D \bar{D}^{*}$ decay width of the $Z_{c}(3900)^{+}$ would be $4 \mathrm{MeV}$, and small compared to that for $\pi^{+} J / \psi$, which they estimated to be $\simeq 29 \mathrm{MeV}$. Subsequently the $D \bar{D}^{*}$ decays have been seen, with a decay with that is $6.2 \pm 2.9$ times larger than that for $\pi^{+} J / \psi[69]$, not six times smaller ${ }^{11)}$.

One attractive feature of the tetraquark feature is that it can explain the large partial width for transitions such as $Y(4260) \rightarrow \pi^{+} \pi^{-} J / \psi, X(3915) \rightarrow \omega J / \psi$, $Z(4430) \rightarrow \pi^{+} \psi^{\prime}$, etc., in a natural way. The corresponding transitions in the charmonium system are OZIsuppressed, and the partial widths are relatively small $\leqslant 100 \mathrm{keV}$. In molecular models, although OZIsuppression can be evaded, the $Q$ and $\bar{Q}$ reside in different constituent mesons with small spatial overlap. In addition they have uncorrelated colors and spins. In contrast, tetraquarks are compact. The the $Q$ and $\bar{Q}$ necessarily have a large overlap and tightly correlated color and spin and the presence of the additional light quarks eliminates OZI suppression. A tetraquark interpretation of the charged bottomoniumlike $Z_{b}(10610)$ and $Z_{b}(10650)$ states is advocated by Ali and collaborators $[172,173]$.

\subsubsection{QCD-hybrids?}

Since $Q \bar{Q}$-gluon hybrid mesons cannot account for any of the charged $Z$ states, their role in explaining the $X Y Z$ meson puzzles described here has to be limited. As mentioned above, there has been a number of papers that identify the $1^{--} Y$ states as charmonium hybrids. In $1^{--}$hybrids, the $Q$ and $\bar{Q}$ are in a relative $P$-wave, which suppresses their $\Gamma_{e^{+} e^{-}}$partial widths. The experimental $90 \%$ CL upper limit $\Gamma_{e^{+} e^{-}}(Y(4260))<580 \mathrm{eV}[45]$ is smaller than corresponding width for the nearby $3^{3} S_{1}$ state: $\Gamma_{e^{+} e^{-}}(\psi(4040))=860 \pm 7 \mathrm{eV}$ and about the same as that for the $4^{3} S_{1}$ state: $\Gamma_{e^{+} e^{-}}(\psi(4415))=580 \pm 70$ $\mathrm{eV}$ [13]. For similar reasons, decays to $S$-wave plus $P$ wave open charmed mesons are supposed to be dominant. As mentioned above in the discussion related to the study of $Z_{c}(3885) \rightarrow D \bar{D}^{*}$ in $Y(4260) \rightarrow \pi D \bar{D}^{*}$ decays, there is no strong evidence for subthreshold $Y(4260) \rightarrow D \bar{D}_{1}(2420)$ decays in these final states [69]. Thus, although there is a lot of theoretical enthusiasm for identifying the $1^{--} Y$ states as $c \bar{c}$-gluon hybrids, there is not much experimental evidence to support this assignment. Perhaps theoretical analyses of the recent BESIII observations of strong decay widths for $Y(4260) \rightarrow \pi^{+} Z_{c}(3900)^{-} \& \pi^{+} Z_{c}(4020)^{-}[49,52]$ and the radiative transition $Y(4260) \rightarrow \gamma X(3872)$ [115] will clarify the situation.

11) Here I assume that the $Z_{c}(3885)$ and $Z_{c}(3900)$ are the same state. 


\subsubsection{Hadrocharmonium?}

Voloshin points out that the $\simeq 23 \mathrm{MeV}$ mass difference between the $Z_{c}(3900)$ (measured in the $\pi^{+} J / \psi$ channel) and the $D^{+} \bar{D}^{* 0}$ (or $D^{*+} \bar{D}^{0}$ ) threshold is not that small [147]. The momenta of the "constituent" $D^{(*)}$ mesons in the $Z_{c}$ restframe would be substantial, $\sim 200 \mathrm{MeV} / \mathrm{c}^{12)}$. Since $J^{P}=1^{+}$, the mesons would be in an $S$-wave with no centrifugal barrier to hold them together, in which case, the total width $29 \pm 10 \mathrm{MeV}^{13}$ ) seems too small. Also, in a $D \bar{D}^{*}$ bound state, the spins of the $c$ and the $\bar{c}$ quarks would be uncorrelated and, thus, the $c \bar{c}$ system should consist of equal amounts of $S=0$ and $S=1$. This suggests that the decays $Z_{c}(3900) \rightarrow \pi J / \psi$ and $\pi h_{c}$ should occur at a similar rate, contrary to the observation reported in Ref. [51]. As discussed above, BESIII measurements show that the $Z_{c}(3900) \rightarrow \pi h_{c}$ rate is suppressed relative to that for the $\pi J / \psi$ mode by at least a factor of five. While the hadrocharmonium model fixes the above problems, it, like the tetraquark model, predicts a lower mass partner state while BESIII found the $Z_{c}(4020)$ at higher mass. Also, the hadrocharmonium prediction for the $D \bar{D}^{*}$ decay width is similar to the 4 $\mathrm{MeV}$ value predicted by the tetraquark model, and is in strong contradiction with experimental observatiions.

\subsubsection{A unified model?}

None of the models discussed above gives a compelling picture of all the observed data. Thus, in our current situation, we are forced to a attribute some of the observed states to one picture and others to a different scenario. In some cases, like the $X(3872)$, mixtures of different models have been invoked. In all cases, these pictures accept the standard potential-model based quark model ideas and apply molecule or tetraquark, etc., ideas to those states where the conventional approach fails. Thus, molecular models work for near-threshold "exotic" states, tetraquarks for scalar mesons, $c \bar{c}$-gluon hybrids for the $1^{--} Y$ states, etc. Since all these approaches have problems, the situation is not very satisfying.

An exception to these piecemeal approaches is an ambitious project by Friedmann that eschews potential model and non-relativistic quantum mechanics ideas altogether and, instead, attempts to build the spectrum of hadrons directly from QCD principles alone, using quark and diquark constituents on an equal footing [174]. With this approach, Friedmann is able to categorize all of the established hadrons as designated by the PDG at the time of her publication, including many of the "exotic" ones listed above, in an elegant concise way. A striking feature of this scheme is that all of the states can be incorporated in her scheme without invoking any radial excitations. In conventional models, radial excitations are essential features but, with them, the model runs into lots of problems, especially with the categorization of the baryon spectrum. For example, the $J^{P}=1 / 2^{+}, N(1440)$ "Roper" resonance, which defies any compelling classification in the quark model, is naturally accommodated in Freidmann's scheme as a $(q q)_{A}(q q)_{A} \bar{q}$ state, where the two asymmetric, spin-zero diquarks $\left((q q)_{A}\right)$ are in a relative $P$-wave. The $\psi^{\prime}$, which is the quintessential radially excited meson in the quark model, is identified as a $(q q)_{A}(\bar{q} \bar{q})_{A}$ pair in a $P$-wave. Of course this is just a clasification scheme and it remains to be seen if a tetraquark picture of the $\psi^{\prime}$ can reproduce the success of the charmonium model description of the $\psi^{\prime}$ properties such as, for example, the ratio of $\Gamma_{e^{+} e^{-}}\left(\psi^{\prime}\right) / \Gamma_{e^{+} e^{-}}(J / \psi)$. Nevertheless, no one could accuse Friedmann of being a "prisoner of conventional thinking." ${ }^{14)}$

\section{Summary}

The QCD exotic states that are much preferred by theorists, such as pentaquarks, the $H$-dibaryon, and meson hybrids with exotic $J^{P C}$ values continue to elude confirmation even in experiments with increasingly high levels of sensitivity. On the other hand, a candidate $p \bar{p}$ bound state and a rich spectroscopy of quarkoniumlike states that do not fit into the remaining unassigned levels for $c \bar{c}$ charmonium and $b \bar{b}$ bottomonium states has emerged. No compelling theoretical picture has yet been found that provides a comprehensive description of what is seen, but, since at least some of these states are near $D^{(*)} \bar{D}^{*}$ or $B^{(*)} \bar{B}^{*}$ thresholds and couple to $S$-wave combinations of these states, molecule-like configurations necessarily have to be important components of their wave functions [112]. This has inspired a new field of "flavor chemistry" that is attracting considerable attention both by the experimental and theoretical hadron physics communities $[29,30]$. With the increased emphasis of the BESIII and LHC experiments on this subject and the imminent operation of BelleII [85], the high luminosity reincarnation

\footnotetext{
12) With my average of all $Z_{c}(3900)$ mass measurements, $\left\langle M_{Z_{C}(3900)}\right\rangle=3890 \pm 3 \mathrm{MeV}$, and the difference between the $Z_{c}(3900)$ mass and the $D^{*}$ mass threshold is lower, at $\simeq 14 \mathrm{MeV}$, and the constituent $D^{(*)}$ momentum in the $Z_{c}$ restframe decreases to $\simeq 170$ $\mathrm{MeV}$.

13) This is my average of the measurements reported in Refs. [49] and [69].

14) http://scitation.aip.org/content/aip/magazine/physicstoday/news/10.1063/PT.5.3012
} 
of Belle, and the future operation of the PANDA experiment at FAIR [175], we can expect a continuous flow of interesting experimental results including, probably, many new surprises and, hopefully, new, groundbreaking insights into the long-distance behavior of QCD.

Acknowledgements While preparing this report I benefited from communications from Eric Braaten, Sookyung Choi, Tamar Friedmann, Pyungwon Ko, Tomasz Skwarnicki, Sachiko Takeuchi, Kai Yi, Qiang Zhao and colleagues on the Belle \& BESIII experiments. This work was supported in part by the Korean National Research Foundation Grant No. 2011-0029457 and the Institute for Basic Science (Korea) Project Code IBS-R016-D1.

Open Access This article is distributed under the terms of the Creative Commons Attribution License which permits any use, distribution, and reproduction in any medium, provided the original author(s) and the source are credited.

\section{References and notes}

1. R. L. Jaffe, $Q^{2} \bar{Q}^{2}$ resonances in the baryon-antibaryon system, Phys. Rev. D 17, 1444 (1978)

2. T. Nakano, et al. (LEPS Collaboration), Evidence for a narrow $S=+1$ baryon resonance in photoproduction from the neutron, Phys. Rev. Lett. 91, 012002 (2003)

3. D. Diakonov, V. Petrov, and M. Polyakov, Exotic antidecuplet of baryons: Prediction from chiral solitons, Z. Phys. A 359, 305 (1997)

4. A review of the events during this period together with references to the experimental work is provided in: R. A. Schumacher, The rise and fall of pentaquarks in experiments, arXiv: nucl-ex/0512042 (2005)

5. See, for example, B. McKinnon, et al. (CLAS Collaboration), Search for the $\Theta^{+}$pentaquark in the reaction $\gamma \mathrm{d} \rightarrow \mathrm{pK}^{-} \mathrm{K}^{+} \mathrm{n}$, Phys. Rev. Lett. 96, 212001 (2006)

6. K. Shirotori, T. N. Takahashi, S. Adachi, M. Agnello, S. Ajimura, et al., Search for the $\Theta^{+}$pentaquark via the $\pi^{-} \mathrm{p} \rightarrow \mathrm{K}^{-} \mathrm{X}$ reaction at $1.92 \mathrm{GeV} / \mathrm{c}$, Phys. Rev. Lett. 109(13), 132002 (2012)(and references cited therein)

7. W.-M. Yao, et al. (Particle Data Group), Review of particle physics, J. Phys. G 33(1), 1 (2006). see, in particular, the "Pentaquark Update" by G. Trilling on page 1019.

8. R. L. Jaffe, Perhaps a stable dihyperon, Phys. Rev. Lett. 38, 195 (1977)

9. H. Takahashi, J. K. Ahn, H. Akikawa, S. Aoki, K. Arai, et al., Observation of a ${ }_{\Lambda}^{6} \mathrm{He}$ double hypernucleus, Phys. Rev. Lett. 87(21), 212502 (2001)

10. S. R. Beane, et al. (NPLQCD Collaboration), Evidence for a bound H dibaryon from lattice QCD, Phys. Rev. Lett. 106, 162001 (2011)

11. T. Inoue, et al. (HALQCD Collaboration), Bound $\mathrm{H}$ dibaryon in flavor $S U(3)$ limit of lattice QCD, Phys. Rev. Lett. 106, 162002 (2011)
12. B. H. Kim, et al. (Belle Collaboration), Search for an Hdibaryon with a mass near $2 m_{\Lambda}$ in $\Upsilon(1 \mathrm{~S})$ and $\Upsilon(2 \mathrm{~S})$ decays, Phys. Rev. Lett. 110, 222002 (2013)

13. J. Beringer, et al. (Particle Data Group), Review of particle physics, Phys. Rev. D 86(1), 010001 (2012)

14. Reported by T. E. Barnes, HADRON05 summary: Heavy quark hadrons and theory, arXiv: hep-ph/0510365 (2005)

15. R. L. Jaffe, Exotica, arXiv: hep-ph/0409065v2 (2004)

16. J. Z. Bai, et al. (BES Collaboration), Observation of a nearthreshold enhancement in the $\bar{p} \overline{\mathrm{p}}$ mass spectrum from radiative $\mathrm{J} / \psi \rightarrow \gamma \mathrm{p} \overline{\mathrm{p}}$ decays, Phys. Rev. Lett. 91, 022001 (2003)

17. E. Fermi and C. N. Yang, Are mesons elementary particles? Phys. Rev. 76(12), 1739 (1949)

18. S. Godfrey and S. L. Olsen, The exotic $X Y Z$ charmoniumlike mesons, Annu. Rev. Nucl. Part. Sci. 58(1), 51 (2008)

19. B. S. Zou and H. C. Chiang, One-pion-exchange final-state interaction and the p $\overline{\mathrm{p}}$ near threshold enhancement in $\mathrm{J} / \psi \rightarrow$

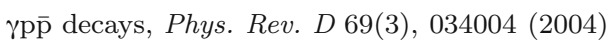

20. A. Sibirtsev, J. Haidenbauer, S. Krewald, U. G. Meißner, and A. Thomas, Near threshold enhancement of the p $\bar{p}$ mass spectrum in J/ $\psi$ decay, Phys. Rev. D 71(5), 054010 (2005)

21. G. J. Ding and M. L. Yan, Proton-antiproton annihilation in baryonium, Phys. Rev. C 72(1), 034014 (2005)

22. M. Ablikim, et al. (BES Collaboration), Observation of a resonance $\mathrm{X}(1835)$ in $\mathrm{J} / \psi \rightarrow \gamma \pi^{+} \pi^{-} \eta^{\prime}$, Phys. Rev. Lett. 95, 262001 (2005)

23. M. Ablikim, et al. (BES Collaboration), Spin-parity analysis of $\bar{p} \overline{\mathrm{p}}$ mass threshold structure in $\mathrm{J} / \psi$ and $\psi(3686)$ radiative decays, Phys. Rev. Lett. 108, 112003 (2012)

24. M. Ablikim, et al. (BES Collaboration), Confirmation of the $\mathrm{X}(1835)$ and observation of the resonances $\mathrm{X}(2120)$ and $\mathrm{X}(2370)$ in $\mathrm{J} / \psi \rightarrow \gamma \pi^{+} \pi^{-} \eta^{\prime}$, Phys. Rev. Lett. 106, 072002 (2011)

25. M. Ablikim, et al. (BES Collaboration), Study of $\mathrm{J} / \psi$ decaying into $\omega \mathrm{p} \overline{\mathrm{p}}$, Eur. Phys. J. C 53, 15 (2008), arXiv: 0710.5369 [hep-ex]

26. S. B. Athar, et al. (CLEO Collaboration), Radiative decays of the $\Upsilon(1 \mathrm{~S})$ to a pair of charged hadrons, Phys. Rev. D 73, 032001 (2006)

27. M.-Z. Wang, et al. (Belle Collaboration), Observation of $\mathrm{B}^{+} \rightarrow \mathrm{p} \overline{\mathrm{p}} \pi^{+}, \mathrm{B}^{0} \rightarrow \mathrm{p} \overline{\mathrm{p}} \mathrm{K}^{0}$, and $\mathrm{B}^{+} \rightarrow \mathrm{p} \overline{\mathrm{p}} \mathrm{K}^{*+}$, Phys. Rev. Lett. 92, 131801 (2004)

28. See, for example, M. Ablikim, et al. (BESIII Collaboration), Observation of a structure at $1.84 \mathrm{GeV} / \mathrm{c}^{2}$ in the $3\left(\pi^{+} \pi^{-}\right)$ mass spectrum in $\mathrm{J} / \psi \rightarrow \gamma 3\left(\pi^{+} \pi^{-}\right)$decays, Phys. Rev. $D$ 88, 091502(R) (2013)

29. For recent reviews see N. Brambilla, S. Eidelman, B. K. Heltsley, R. Vogt, G. T. Bodwin, et al., Heavy quarkonium: Progress, puzzles, and opportunities, Eur. Phys. J. C 71(2), 1534 (2011)

30. G. T. Bodwin, E. Braaten, E. Eichten, S. L. Olsen, T. K. Pedlar, and J. Russ, Quarkonium at the frontiers of high energy physics: A snowmass white paper, arXiv: 1307.7425 [hep-ph] (2013) 
31. K. Abe, et al. (Belle Collaboration), Observation of a charmoniumlike state produced in association with a $\mathrm{J} / \psi$ in $\mathrm{e}^{+} \mathrm{e}^{-}$annihilation at $\sqrt{s} \approx 10.6 \mathrm{GeV}$, Phys. Rev. Lett. 98, 082001 (2007)

32. P. Pakhlov, et al. (Belle Collaboration), Production of new charmoniumlike states in $\mathrm{e}^{+} \mathrm{e}^{-} \rightarrow \mathrm{J} / \psi \mathrm{D}^{*} \overline{\mathrm{D}}^{*}$ at $\sqrt{s} \approx 10.6$ GeV, Phys. Rev. Lett. 100, 202001 (2008)

33. K.-T. Chao, Interpretations for the observed in the double charm production at B factories, Phys. Lett. B 661(5), 348 (2008)

34. J.-Z. Bai, et al. (BES Collaboration), Measurements of the cross section for $\mathrm{e}^{+} \mathrm{e}^{-} \rightarrow$ hadrons at center-of-mass energies from 2 to $5 \mathrm{GeV}$, Phys. Rev. Lett. 88, 101802 (2002)

35. M. Ablikim, et al. (BES Collaboration), Determination of the $\psi(3770), \psi(4040), \psi(4160)$ and $\psi(4415)$ resonance parameters, Phys. Lett. B 660(4), 315 (2008)

36. S. Godfrey and N. Isgur, Mesons in a relativized quark model with chromodynamics, Phys. Rev. D 32(1), 189 (1985)

37. B. Aubert, et al. (BaBar Collaboration), Observation of a broad structure in the $\pi^{+} \pi^{-} \mathrm{J} / \psi$ mass spectrum around 4.26 $\mathrm{GeV} / \mathrm{c}^{2}$, Phys. Rev. Lett. 95, 142001 (2005)

38. B. Aubert, et al. (BaBar Collaboration), Evidence of a broad structure at an invariant mass of $4.32 \mathrm{GeV} / \mathrm{c}^{2}$ in the reaction $\mathrm{e}^{+} \mathrm{e}^{-} \rightarrow \pi^{+} \pi^{-} \psi(2 \mathrm{~S})$ measured at BABAR, Phys. Rev. Lett. 98, 212001 (2007)

39. C. Z. Yuan, et al. (Belle Collaboration), Measurement of the $\mathrm{e}^{+} \mathrm{e}^{-} \rightarrow \pi^{+} \pi^{-} \mathrm{J} / \psi$ cross section via initial-state radiation at Belle, Phys. Rev. Lett. 99, 182004 (2007)

40. X. L. Wang, et al. (Belle Collaboration), Observation of two resonant structures in $\mathrm{e}^{+} \mathrm{e}^{-} \rightarrow \pi^{+} \pi^{-} \psi(2 \mathrm{~S})$ via initial-state radiation at Belle, Phys. Rev. Lett. 99, 142002 (2007)

41. G. Pakhlova, et al. (Belle Collaboration), Measurement of the near-threshold $\mathrm{e}^{+} \mathrm{e}^{-} \rightarrow \mathrm{D}^{(*) \pm} \mathrm{D}^{(*)} \mp$ cross section using initial-state radiation, Phys. Rev. Lett. 98, 092001 (2007)

42. G. Pakhlova, et al. (Belle Collaboration), Observation of the $\psi(4415) \rightarrow \mathrm{D} \bar{D}_{2}^{*}(2460)$ decay using initial-state radiation, Phys. Rev. Lett. 100, 062001 (2008)

43. G. Pakhlova, et al. (Belle Collaboration), Observation of a near-threshold enhancement in the $\mathrm{e}^{+} \mathrm{e}^{-} \rightarrow \Lambda_{c}^{+} \Lambda_{c}^{-}$cross section using initial-state radiation, Phys. Rev. Lett. 101, 172001 (2008)

44. G. Pakhlova, et al. (Belle Collaboration), Measurement of the $\mathrm{e}^{+} \mathrm{e}^{-} \rightarrow \mathrm{D}^{0} \mathrm{D}^{*-} \pi^{+}$cross section using initial-state radiation, Phys. Rev. D 80, 091101(R) (2009)

45. X. H. Mo, G. Li, C. Z. Yuan, K. L. He, H. M. Hu, J. H. Hu, P. Wang, and Z. Y. Wang, Determining the upper limit of $\Gamma_{e e}$ for the Y(4260), Phys. Lett. B 640(4), 182 (2006)

46. See, for example, S.-L. Zhu, The possible interpretations of Y(4260), Phys. Lett. B 625(3-4), 212 (2005)

47. E. Kou and O. Pene, Suppressed decay into open charm for the being a hybrid, Phys. Lett. B 631(4), 164 (2005)

48. Q. Wang, C. Hanhart, and Q. Zhao, Decoding the riddle of $\mathrm{Y}(4260)$ and $\mathrm{Z}_{c}(3900)$, Phys. Rev. Lett. 111(13), 132003 (2013)
49. M. Ablikim, et al. (BESIII Collaboration), Observation of a charged charmoniumlike structure in $\mathrm{e}^{+} \mathrm{e}^{-} \rightarrow \pi^{+} \pi^{-} \mathrm{J} / \psi$ at $\sqrt{s}=4.26 \mathrm{GeV}$, Phys. Rev. Lett. 110, 252001 (2013)

50. Z. Q. Liu, et al. (Belle Collaboration), Study of $\mathrm{e}^{+} \mathrm{e}^{-} \rightarrow$ $\pi^{+} \pi^{-} \mathrm{J} / \psi$ and observation of a charged charmoniumlike state at Belle, Phys. Rev. Lett. 110, 252002 (2013)

51. M. Ablikim, et al. (BESIII Collaboration), Observation of a charged charmoniumlike structure $\mathrm{Z}_{c}(4020)$ and search for the $\mathrm{Z}_{c}(3900)$ in $\mathrm{e}^{+} \mathrm{e}^{-} \rightarrow \pi^{+} \pi^{-}$hc, Phys. Rev. Lett. 111, 242001 (2013)

52. M. Ablikim, et al. (BESIII Collaboration), Observation of a charged charmoniumlike structure in $\mathrm{e}^{+} \mathrm{e}^{-} \rightarrow\left(\mathrm{D}^{*} \overline{\mathrm{D}}^{*}\right)^{ \pm} \pi^{\mp}$ at $\sqrt{s}=4.26 \mathrm{GeV}$, Phys. Rev. Lett. 112, 132001 (2014), arXiv: 1308.2760 [hep-ex]

53. R. Mizuk, et al. (Belle Collaboration), Observation of two resonance-like structures in the $\pi^{+} \chi_{c 1}$ mass distribution in exclusive $\overline{\mathrm{B}}^{0} \rightarrow \mathrm{K}^{-} \pi^{+} \chi_{c 1}$ decays, Phys. Rev. D 78, 072004 (2008)

54. S.-K. Choi, et al. (Belle Collaboration), Observation of a resonancelike structure in the $\pi^{ \pm} \psi^{\prime}$ mass distribution in exclusive $\mathrm{B} \rightarrow \mathrm{K} \pi \pi^{ \pm} \psi^{\prime}$ decays, Phys. Rev. Lett. 100, 142001 (2008)

55. B. Aubert, et al. (BaBar Collaboration), Search for the $\mathrm{Z}(4430)^{-}$at BABAR, Phys. Rev. D 79, 112001 (2009)

56. R. Mizuk, et al. (Belle Collaboration), Dalitz analysis of $\mathrm{B} \rightarrow \mathrm{K} \pi^{+} \psi^{\prime}$ decays and the $\mathrm{Z}(4430)^{+}$, Phys. Rev. D 80, 031104(R) (2009)

57. K. Chilikin, et al. (Belle Collaboration), Experimental constraints on the spin and parity of the $\mathrm{Z}(4430)^{+}$, Phys. Rev. D 88, 074026 (2013)

58. R. Aaij, et al. (LHCb Collaboration), Observation of the resonant character of the $\mathrm{Z}(4430)^{-}$state, Phys. Rev. Lett. 112, 222002 (2014)

59. See, for example, P. Pakhlov, Charged charmonium-like states as rescattering effects in decays, Phys. Lett. B 702(23), 139 (2011)

60. P. Pakhlov and T. Uglov, Charged charmonium-like $\mathrm{Z}^{+}$(4430) from rescattering in conventional B decays, arXiv: 1408.5295 [hep-ph] (2014)

61. B. Aubert, et al. (BaBar Collaboration), Measurements of the absolute branching fractions of $\mathrm{B}^{ \pm} \rightarrow \mathrm{K}^{ \pm} \mathrm{X}_{\mathrm{c} \overline{\mathrm{c}}}$, Phys. Rev. Lett. 96, 052002 (2006)

62. K. Chilikin, et al. (Belle Collaboration), Observation of a new charged charmonium-like state in $\mathrm{B} \rightarrow \mathrm{J} / \psi \mathrm{K} \pi$ decays, arXiv: 1408.6457 (2014) (submitted for publication in Phys. Rev. D)

63. For discussions of factorization in heavy quark decays see, for example, M. Beneke, G. Buchelle, M. Neubert, and C. Sachrajda, QCD factorization for $\mathrm{B} \rightarrow \pi \pi$ decays: Strong phases and CP violation in the heavy quark limit, Phys. Rev. Lett. 83(10), 1914 (1999)

64. M. Beneke, G. Buchelle, M. Neubert, and C. Sachrajda, QCD factorization for exclusive non-leptonic -meson decays: General arguments and the case of heavy-light final states, Nucl. Phys. B 591(1-2), 313 (2000) 
65. M. Beneke, G. Buchelle, M. Neubert, and C. Sachrajda, QCD factorization in $\mathrm{B} \rightarrow \pi \mathrm{K}, \pi \pi$ decays and extraction of Wolfenstein parameters, arXiv: hep-ph/0104110 (2001)

66. J. P. Lees, et al. (BaBar Collaboration), Search for the $\mathrm{Z}_{1}(4050)^{+}$and $\mathrm{Z}_{2}(4250)^{+}$states in $\overline{\mathrm{B}}^{0} \rightarrow \chi_{c 1} \mathrm{~K}^{-} \pi^{+}$and $\mathrm{B}^{+} \rightarrow \chi_{c 1} \mathrm{~K}_{\mathrm{S}}^{0} \pi^{+}$, Phys. Rev. D 85, 052003 (2012)

67. C. Zhang, Studies on BEPC upgrade from pretzel to doublering, Sci. China G 53(11), 2084 (2010)

68. M. Ablikim, et al. (BESIII Collaboration), Design and construction of the BESIII detector, Nucl. Instrum. Methods A 614, 345 (2010), arXiv: 0911.4960 [physics.ins-det]

69. M. Ablikim, et al. (BESIII Collaboration), Observation of a charged $\left(\mathrm{DD}^{*}\right)^{ \pm}$mass peak in $\mathrm{e}^{+} \mathrm{e}^{-} \rightarrow \pi \mathrm{DD} \overline{\mathrm{D}}^{*}$ at $\sqrt{s}=4.26$ GeV, Phys. Rev. Lett. 112, 022001 (2014)

70. M. Ablikim, et al. (BESIII Collaboration), Observation of $\mathrm{e}^{+} \mathrm{e}^{-} \rightarrow \pi^{0} \pi^{0} h_{c}$ and a neutral charmoniumlike structure $\mathrm{Z}_{c}(4020)^{0}$, (in preparation) (2014)

71. S. K. Choi, et al. (Belle Collaboration), Observation of a near-threshold $\omega \mathrm{J} / \psi$ mass enhancement in exclusive $\mathrm{B} \rightarrow \mathrm{K} \omega \mathrm{J} / \psi$ decays, Phys. Rev. Lett. 94, 182002 (2005)

72. P. del Amo Sanchez, et al. (BaBar Collaboration), Evidence for the decay $\mathrm{X}(3872) \rightarrow \mathrm{J} / \psi \omega$, Phys. Rev. D 82, 011101(R) (2010)

73. B. Aubert, et al. (BaBar Collaboration), Observation of $\mathrm{Y}(3940) \rightarrow \mathrm{J} / \psi \omega$ in $\mathrm{B} \rightarrow \mathrm{J} / \psi \omega \mathrm{K}$ at $B A B A R$, Phys. Rev. Lett. 101, 082001 (2008)

74. S. Uehara, et al. (Belle Collaboration), Observation of a charmoniumlike enhancement in the $\gamma \gamma \rightarrow \omega \mathrm{J} / \psi$ process, Phys. Rev. Lett. 104, 092001 (2010)

75. J. P. Lees, et al. (BaBar Collaboration), Study of $\mathrm{X}(3915) \rightarrow \mathrm{J} / \psi \omega$ in two-photon collisions, Phys. Rev. D 86, $072002(2012)$

76. T. Barnes, S. Godfrey, and E. S. Swanson, Higher charmonia, Phys. Rev. D 72(5), 054026 (2005)

77. F. K. Guo, and U. G. Meissner, Where is the $\chi_{c 0}(2 \mathrm{P})$ ? Phys. Rev. D 86(9), 091501 (2012)

78. S. Uehara, et al. (Belle Collaboration), Observation of a $\chi_{c 2}^{\prime}$ candidate in $\gamma \gamma \rightarrow \mathrm{D} \overline{\mathrm{D}}$ production at Belle, Phys. Rev. Lett. 96, 082003 (2006)

79. B. Aubert, et al. (BaBar Collaboration), Observation of the $\chi_{c 2}(2 \mathrm{P})$ meson in the reaction $\gamma \gamma \rightarrow \mathrm{D} \overline{\mathrm{D}}$ at $B A B A R$, Phys. Rev. D 81, 092003 (2010)

80. J. Brodzicka, et al. (Belle Collaboration), Observation of a new $\mathrm{D}_{\mathrm{sJ}}$ meson in $\mathrm{B}^{+} \rightarrow \overline{\mathrm{D}}^{0} \mathrm{D}^{0} \mathrm{~K}^{+}$decays, Phys. Rev. Lett. 100, 092001 (2008)

81. B. Aubert, et al. (BaBar Collaboration), Study of resonances in exclusive $\mathrm{B}$ decays to $\mathrm{D}^{-}\left({ }^{*}\right) \mathrm{D}\left({ }^{*}\right) \mathrm{K}$, Phys. Rev. D 77, 011102(R) (2008)

82. This is the weighted average of results reported in Refs. [74] and [75].

83. Y. Jiang, G. L. Wang, T. H. Wang, and W. L. Ju, Why $\mathrm{X}(3915)$ is so narrow as a $\chi_{c 0}(2 \mathrm{P})$ state, arXiv: 1310.2317 [hep-ph] (2013)
84. Y. C. Yang, Z. Xia, and J. Ping, Are the X(4160) and X(3915) charmonium states? Phys. Rev. D 81(9), 094003 (2010)

85. T. Abe, et al. (BelleII Collaboration), Belle II technical design report, arXiv: 1011.0352 [hep-ex] (2010)

86. R. Molina and E. Oset, Y(3940), Z(3930), and the X(4160) as dynamically generated resonances from the vector-vector interaction, Phys. Rev. D 80(11), 114013 (2009)

87. T. Aaltonen, et al. (CDF Collaboration), Evidence for a narrow near-threshold structure in the $J / \psi \phi$ mass spectrum in $\mathrm{B}^{+} \rightarrow \mathrm{J} / \psi \phi \mathrm{K}^{+}$decays, Phys. Rev. Lett. 102, 242002 (2009)

88. T. Aaltonen, et al. (CDF Collaboration), Observation of the $Y(4140)$ structure in the $J / \psi \phi$ mass spectrum in $\mathrm{B}^{ \pm} \rightarrow$ $\mathrm{J} / \psi \phi \mathrm{K}^{ \pm}$decays, arXiv: 1101.6058 (2011)

89. R. Aaij, et al. (LHCb Collaboration), Search for the $X(4140)$ state in $\mathrm{B}^{+} \rightarrow \mathrm{J} / \psi \phi \mathrm{K}^{+}$decays, Phys. Rev. D 85, 091103(R) (2012)

90. S. Chatrchyan, et al. (CMS Collaboration), Observation of a peaking structure in the $\mathrm{J} / \psi \phi$ mass spectrum from $\mathrm{B}^{ \pm} \rightarrow$ $\mathrm{J} / \psi \mathrm{K}^{ \pm}$decays, Phys. Lett. B 734, 261 (2014)

91. J. P. Lees, et al. (BaBar Collaboration), Study of $\mathrm{B}^{ \pm, 0} \rightarrow$ $\mathrm{J} / \psi \mathrm{K}^{+} \mathrm{K}^{-} \mathrm{K}^{ \pm, 0}$ and search for $\mathrm{B}^{0} \rightarrow \mathrm{J} / \psi \phi$ at BABAR, arXiv: 1407.7244, 2014 (submitted for publication in Phys. Rev. D)

92. X. Liu, Z. G. Luo, and S. L. Zhu, Novel charmoniumlike structures in the invariant mass spectra, Phys. Lett. B 699(5), 341 (2011)

93. K. Yi, Experimental review of structures in the $\mathrm{J} / \psi \phi$ mass spectrum, Int. J. Mod. Phys. A 28(18), 1330020 (2013)

94. C. P. Shen, et al. (Belle Collaboration), Evidence for a new resonance and search for the $Y(4140)$ in the $\gamma \gamma \rightarrow \phi \mathrm{J} / \psi$ process, Phys. Rev. Lett. 104, 112004 (2010)

95. S. K. Choi, et al. (Belle Collaboration), Observation of a narrow charmoniumlike state in exclusive $\mathrm{B}^{ \pm} \rightarrow \mathrm{K}^{ \pm} \pi^{+} \pi^{-} \mathrm{J} / \psi$ Decays, Phys. Rev. Lett. 91, 262001 (2003)

96. D. Acosta, et al. (CDF II Collaboration), Observation of the narrow state $\mathrm{X}(3872) \rightarrow \mathrm{J} / \psi \pi^{+} \pi^{-}$in $\mathrm{p} \overline{\mathrm{p}}$ collisions at $\sqrt{s}=1.96 \mathrm{TeV}$, Phys. Rev. Lett. 93, 072001 (2004)

97. V. M. Abazov, et al. (D0 Collaboration), Observation and

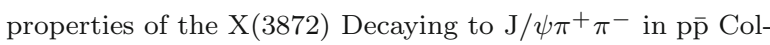
lisions at $\sqrt{s}=1.96 \mathrm{TeV}$, Phys. Rev. Lett. 93, 162002 (2004)

98. B. Aubert, et al. (BaBar Collaboration), Study of the $\mathrm{B}^{-} \rightarrow \mathrm{J} / \psi \mathrm{K}^{-} \pi^{+} \pi^{-}$decay and measurement of the $\mathrm{B}^{-} \rightarrow \mathrm{X}(3872) \mathrm{K}^{-}$branching fraction, Phys. Rev. D 71, 071103 (2005)

99. R. Aaij, et al. (LHCb Collaboration), Observation of $X(3872)$ production in $p p$ collisions at $\sqrt{s}=7 \mathrm{TeV}$, Eur. Phys. J. C 72, 1972 (2012)

100. S. Chatrchyan, et al. (CMS Collaboration), Measurement of the $X(3872)$ production cross section via decays to $\mathrm{J} / \psi \pi \pi$ in pp collisions at $\sqrt{s}=7 \mathrm{TeV}$, J. High Energy Phys. 154, 1304 (2013); see also arXiv: 1302.3968 [hep-ex]

101. S. Eidelman, Talk at the $\phi$ to $\psi$ symposium, Rome, Sep. 9-12, 2013 
102. S.-K. Choi, et al. (Belle Collaboration), Bounds on the width, mass difference and other properties of $\mathrm{X}(3872) \rightarrow$ $\pi^{+} \pi^{-} \mathrm{J} / \psi$ decays, Phys. Rev. D 84, 052004 (2011)

103. R. Aaij, et al. (LHCb Collaboration), Determination of the $\mathrm{X}(3872)$ meson quantum numbers, Phys. Rev. Lett. 110, 222001 (2013)

104. A. Abulencia, et al. (CDF Collaboration), Analysis of the quantum numbers $\mathrm{J}^{\mathrm{PC}}$ of the $X(3872)$ particle, Phys. Rev. Lett. 98, 132002 (2007)

105. D. Acosta, et al. (CDF Collaboration), Measurement of the dipion mass spectrum in $\mathrm{X}(3872) \rightarrow \mathrm{J} / \psi \pi^{+} \pi^{-}$decays, Phys. Rev. Lett. 96, 102002 (2006)

106. B. Aubert, et al. (BaBar Collaboration), Search for a charged partner of the $\mathrm{X}(3872)$ in the $\mathrm{B}$ meson decay $\mathrm{B} \rightarrow \mathrm{X}^{-} \mathrm{K}, \mathrm{X}^{-} \rightarrow \mathrm{J} / \psi \pi^{-} \pi^{0}$, Phys. Rev. D 71, 031501 (2005)

107. K. Abe, et al. (Belle Collaboration), Evidence for $\mathrm{X}(3872$ ) $\rightarrow \gamma \mathrm{J} / \psi$ and the sub-threshold decay $\mathrm{X}(3872) \rightarrow \omega \mathrm{J} / \psi$, arXiv: 0505037 [hep-ex] (2005)

108. T. Aushev, et al. (Belle Collaboration), Study of the $\mathrm{B} \rightarrow \mathrm{X}(3872)\left(\rightarrow \mathrm{D}^{* 0} \overline{\mathrm{D}}^{0}\right) \mathrm{K}$ decay, Phys. Rev. D 81, 031103(R) (2010)

109. G. Gokhroo, et al. (Belle Collaboration), Observation of a near-threshold $\mathrm{D}^{0} \overline{\mathrm{D}}^{0} \pi^{0}$ enhancement in $\mathrm{B} \rightarrow \mathrm{D}^{0} \overline{\mathrm{D}}^{0} \pi^{0} \mathrm{~K}$ decay, Phys. Rev. Lett. 97, 162002 (2006)

110. B. Aubert, et al. (BABAR Collaboration), Study of resonances in exclusive $B$ decays to $\mathrm{D}^{(*)} \overline{\mathrm{D}}^{(*)} \mathrm{K}$, Phys. Rev. $D$ 77, 011102(R) (2008)

111. E. Braaten and M. Lu, Line shapes of the X(3872), Phys. Rev. D 76(9), 094028 (2007)

112. E. Braaten and H. W. Hammmer, Universality in few-body systems with large scattering length, Phys. Rep. 428(5-6), 259 (2006)

113. S. Coito, G. Rupp, and E. van Beveren, $\mathrm{X}(3872)$ is not a true molecule, Eur. Phys. J. C 73(3), 2351 (2013)

114. N. A. Törnqvist, Isospin breaking of the narrow charmonium state of Belle at $3872 \mathrm{MeV}$ as a deuson, Phys. Lett. B 590(3-4), 209 (2004)

115. M. Ablikim, et al. (BESIII Collaboration), Observation of $\mathrm{e}^{+} \mathrm{e}^{-} \rightarrow \gamma \mathrm{X}(3872)$ at BESIII, Phys. Rev. Lett. 112, 092001 (2014)

116. N. A. Törnqvist, From the deuteron to deusons, an analysis of deuteronlike meson-meson bound states, Z. Phys. C 61(3), 525 (1994)

117. N. A. Törnqvist, Comment on the narrow charmonium state of Belle at $3871.8 \mathrm{MeV}$ as a deuson, arXiv: hep-ph/0308277 (2003)

118. See, for example, F. E. Close, and P. R. Page, The threshold resonance, Phys. Lett. B 578(1-2), 119 (2003)

119. C. Y. Wong, Molecular states of heavy quark mesons, Phys. Rev. C 69(5), 055202 (2004)

120. S. Pakvasa and M. Suzuki, On the hidden charm state at $3872 \mathrm{MeV}$, Phys. Lett. B 579(1-2), 67 (2004)
121. E. Braaten and M. Kusunoki, Production of the X(3870) at the $\Upsilon(4 \mathrm{~S})$ by the coalescence of charm mesons, Phys. Rev. D 69(11), 114012 (2004)

122. E. S. Swanson, Short range structure in the X(3872), Phys. Lett. B 588(3-4), 189 (2004)

123. M. B. Voloshin, Heavy quark spin selection rule and the properties of the X(3872), Phys. Lett. B 604(1-2), 69 (2004)

124. S. Fleming, M. Kusunoki, T. Mehan, and U. van Kolck, Pion interactions in the X(3872), Phys. Rev. D 76(3), 034006 (2007)

125. E. Braaten and M. Lu, Line shapes of the X(3872), Phys. Rev. D 76(9), 094028 (2007)

126. S. L. Zhu, New hadron states, Int. J. Mod. Phys. E 17(02), 283 (2009)

127. D. Gamermann and E. Oset, Isospin breaking effects in the X(3872) resonance, Phys. Rev. D 80(1), 014003 (2009)

128. D. Gamermann and E. Oset, Couplings in coupled channels versus wave functions: Application to the $\mathrm{X}(3872)$ resonance, Phys. Rev. D 81(1), 014029 (2010)

129. O. Zhang, C. Meng, and H. Q. Zheng, Ambiversion of X(3872), Phys. Lett. B 680(5), 453 (2009)

130. M. B. Voloshin and L. B. Okun, Hadron molecules and charmonium atom, JETP Lett. 23, 333 (1976)

131. M. Bander, G. L. Shaw, P. Thomas, and S. Meshkov, Exotic mesons and $\mathrm{e}^{+} \mathrm{e}^{-}$annihilation, Phys. Rev. Lett. 36(13), 695 (1976)

132. A. De Rujula, H. Georgi, and S. L. Glashow, Molecular charmonium: A new spectroscopy? Phys. Rev. Lett. 38(7), 317 (1977)

133. A. V. Manohar and M. B. Wise, Exotic states in QCD, Nucl. Phys. B 339(1), 17 (1993)

134. CDF note 7159, http://www-cdf.fnal.gov/physics/new/ bottom/051020.blessed-X3872/XLife/xlonglivedWWW.ps

135. S. Chatrchyan, et al. (CMS Collaboration), Measurement of the $\mathrm{X}(3872)$ production cross section via decays to $\mathrm{J} / \psi \pi \pi$ in pp collisions at $\sqrt{s}=7 \mathrm{TeV}$, J. High Energy Phys. 1304, 154 (2013)

136. C. Bignamini, B. Grinstein, F. Piccinini, A. D. Polosa, and C. Sabelli, Is the $\mathrm{X}(3872)$ production cross section at $\sqrt{s}$ $=1.96 \mathrm{TeV}$ compatible with a hadron molecule interpretation? Phys. Rev. Lett. 103(16), 162001 (2009)

137. B. Aubert, et al. (BaBar Collaboration), Evidence for $\mathrm{X}(3872) \rightarrow \psi(2 \mathrm{~S}) \gamma$ in $\mathrm{B}^{ \pm} \rightarrow \mathrm{X}(3872) \mathrm{K}^{ \pm}$decays and a study of $\mathrm{B} \rightarrow \mathrm{c} \overline{\mathrm{c}} \gamma \mathrm{K}$, Phys. Rev. Lett. 102, 132001 (2009)

138. V. Bhardwaj, et al. (Belle Collaboration), Observation of $\mathrm{X}(3872) \rightarrow \mathrm{J} / \psi \gamma$ and search for $\mathrm{X}(3872) \rightarrow \psi^{\prime} \gamma$ in B decays, Phys. Rev. Lett. 107, 091803 (2011)

139. F. Aceti, R. Molina, and E. Oset, $\mathrm{X}(3872) \rightarrow \mathrm{J} / \psi \gamma$ decay in the $\mathrm{D}^{*}$ molecular picture, Phys. Rev. D 86(11), 113007 (2012)

140. E. S. Swanson, Diagnostic decays of the X(3872), Phys. Lett. B 598(3-4), 197 (2004)

141. R. Aaij, et al. (LHCb Collaboration), Evidence for the decay $\mathrm{X}(3872) \rightarrow \psi(2 \mathrm{~S}) \gamma$, Nucl. Phys. B 886, 665 (2014) 
142. L. Maiani, F. Piccinini, A. D. Polosa, and V. Riguer, Diquark-antidiquark states with hidden or open charm and the nature of X(3872), Phys. Rev. D 71(1), 014028 (2005)

143. L. Maiani, V. Riguer, R. Faccini, F. Piccinini, A. Pilloni and A. D. Polosa, $\mathrm{J}^{\mathrm{PC}}=1^{++}$charged resonance in the $\Upsilon(4260) \rightarrow \pi^{+} \pi^{-} \mathrm{J} / \psi$ decay? Phys. Rev. D 87, 111102(R) (2013)

144. L. Maiani, F. Piccinini, A. D. Polosa, and V. Riguer, Z(4430) and a new paradigm for spin interactions in tetraquarks, Phys. Rev. D 89(11), 114010 (2014)

145. D. Horn and H. Mandula, Model of mesons with constituent gluons, Phys. Rev. D 17(3), 898 (1978)

146. L. Liu, et al. (Hadron Spectrum Collaboration), Excited and exotic charmonium spectroscopy from lattice QCD, J. High Energy Phys. 07, 126 (2012)

147. M. B. Voloshin, $\mathrm{Z}_{c}(3900)$-what is inside? arXiv: 1304.0380 (2013)

148. M. Takizawa and S. Takeuchi, X(3872) as a hybrid state of the charmonium and the hadronic molecule, Prog. Theor. Exp. Phys. 9, 093D01, (2013), arXiv: 1206.4877

149. K. K. Seth, The quintessential exotic X(3872), Prog. Part. Nucl. Phys. 67(2), 390 (2012)

150. W. S. Hou, Searching for the bottom counterparts of X(3872) and Y(4260) via $\pi^{+} \pi^{-\Upsilon}$, Phys. Rev. D 74(1), 017504 (2006)

151. K.-F. Chen, et al. (Belle Collaboration), Observation of an enhancement in $\mathrm{e}^{+} \mathrm{e}^{-} \rightarrow \Upsilon(1 \mathrm{~S}) \pi^{+} \pi^{-}, \Upsilon(2 \mathrm{~S}) \pi^{+} \pi^{-}$, and $\Upsilon(3 \mathrm{~S}) \pi^{+} \pi^{-}$production near $\sqrt{s}=10.89 \mathrm{GeV}$, Phys. Rev. $D$ 82, 091106(R) (2010)

152. K.-F. Chen, et al. (Belle Collaboration), Observation of anomalous $\Upsilon(1 \mathrm{~S}) \pi^{+} \pi^{-}$and $\Upsilon(2 \mathrm{~S}) \pi^{+} \pi^{-}$production near the $\Upsilon(5 S)$ Resonance, Phys. Rev. Lett. 100, 112001 (2008)

153. A. Ali, C. Hambrock, I. Ahmed, and M. J. Aslam, A case for hidden $\mathrm{b} \overline{\mathrm{b}}$ tetraquarks based on $\mathrm{e}^{+} \mathrm{e}^{-} \rightarrow \mathrm{b} \overline{\mathrm{b}}$ cross section between $\sqrt{s}=10.54$ and $11.20 \mathrm{GeV}$, Phys. Lett. B 684(1), $28(2010)$

154. I. Adachi, et al. (Belle Collaboration), First observation of the $\mathrm{P}$-wave spin-singlet bottomonium states $\mathrm{h}_{\mathrm{b}}(1 \mathrm{P})$ and $\mathrm{h}_{\mathrm{b}}(2 \mathrm{P})$, Phys. Rev. Lett. 108, 032001 (2012)

155. A. Bondar, et al. (Belle Collaboration), Observation of Two Charged Bottomoniumlike Resonances in $\Upsilon(5 \mathrm{~S})$ Decays, Phys. Rev. Lett. 108, 122001 (2012)

156. A. Bondar, Talk at the $\phi$ to $\psi$ symposium, Rome, Sep. 9-12, 2013

157. P. Krokovny, et al. (Belle Collaboration), First observation of the $\mathrm{Z}_{\mathrm{b}}^{0}(10610)$ in a Dalitz analysis of $\Upsilon(10860) \rightarrow$ $\Upsilon(n \mathrm{~S}) \pi^{0} \pi^{0}$, Phys. Rev. D 88, 052016 (2013)

158. A. Garmash, et al. (Belle Collaboration), Amplitude analysis of $\mathrm{e}^{+} \mathrm{e}^{-} \rightarrow \Upsilon(n \mathrm{~S}) \pi^{+} \pi^{-}$at $\sqrt{s}=10.865 \mathrm{GeV}$, arXiv: 1403.0992 [hep-ex] (submitted for publication in Phys. Rev. D)

159. I. Adachi, et al. (Belle Collaboration), Observation of two charged bottomonium-like resonances, arXiv: 1105.4583 (2011)
160. I. Adachi, et al. (Belle Collaboration), Study of three-body Y(10860) decays, arXiv: 1209.6450 (2012)

161. S. Eidelman, B. K. Heltsley, J. J. Hernandez-Rey, S. Navas, and C. Patrignani, Developments in heavy quarkonium spectroscopy, arXiv: 1205.4189 [hep-ex] (2012)

162. T. Aaltonen, et al. (CDF Collaboration), Precision measurement of the $\mathrm{X}(3872)$ mass in $\mathrm{J} / \psi \pi^{+} \pi^{-}$decays, Phys. Rev. Lett. 103, 152001 (2009)

163. B. Aubert, et al. (BaBar Collaboration), Study of the exclusive initial-state-radiation production of the DD system, Phys. Rev. D 76, 111105(R) (2007)

164. G. Pakhlova, et al. (Belle Collaboration), Measurement of the near-threshold $\mathrm{e}^{+} \mathrm{e}^{-} \rightarrow \mathrm{D} \overline{\mathrm{D}}$ cross section using initialstate radiation, Phys. Rev. D 77, 011103(R) (2008)

165. B. Aubert, et al. (BaBar Collaboration), Study of the $\pi^{+} \pi^{-} \mathrm{J} / \psi$ mass spectrum via initial-state radiation at BABAR, arXiv: 0808.1543v2 [hep-ex] (2008)

166. Q. He, et al. (CLEO Collaboration), Confirmation of the $\mathrm{Y}(4260)$ resonance production in initial state radiation, Phys. Rev. D 74, 091104(R) (2006)

167. T. E. Coan, et al. (CLEO Collaboration), Charmonium decays of $\mathrm{Y}(4260), \psi(4160)$, and $\psi(4040)$, Phys. Rev. Lett. 96, 162003 (2006)

168. G. Pakhlova, et al, Belle Collaboration, Observation of a near-threshold enhancement in the $\mathrm{e}^{+} \mathrm{e}^{-} \rightarrow \Lambda_{\mathrm{c}}^{+} \Lambda_{\mathrm{c}}^{-}$cross section using initial-state radiation, Phys. Rev. Lett. 101, 172001 (2008)

169. P. del Amo Sanchez, et al. (BaBar Collaboration), Measurement of the $\mathrm{B} \rightarrow \overline{\mathrm{D}}^{(*)} \mathrm{D}^{(*)} \mathrm{K}$ branching fractions, Phys. Rev. D 83, 032004 (2011)

170. P. del Amo Sanchez, et al. (BaBar Collaboration), Observation of new resonances decaying to $\mathrm{D} \pi$ and $\mathrm{D}^{*} \pi$ in inclusive $\mathrm{e}^{+} \mathrm{e}^{-}$collisions near $\sqrt{s}=10.58 \mathrm{GeV}$, Phys. Rev. D 82, 111101(R) (2010)

171. S. Chatrchyan, et al. (CMS Collaboration), Search for a new bottomonium state decaying to $\Upsilon(1 \mathrm{~S}) \pi^{+} \pi^{-}$in pp collisions at $\sqrt{s}=8 \mathrm{TeV}$, Phys. Lett. B 727, 57 (2013)

172. A. Ali, C. Hambrock, and W. Wang, Tetraquark interpretation of the charged bottomonium-like states $Z_{b}^{ \pm}(10610)$ and $\mathrm{Z}_{b}^{ \pm}(10650)$ and implications, Phys. Rev. D 85(5), 054011 (2013)

173. A. Ali, C. Hambrock, and M. J. Aslam, Tetraquark interpretation of the BELLE data on the anomalous $\Upsilon(1 \mathrm{~S}) \pi^{+} \pi^{-}$ and $\Upsilon(2 \mathrm{~S}) \pi^{+} \pi^{-}$production near the $\Upsilon(5 \mathrm{~S})$ resonance, Phys. Rev. Lett. 104(16), 162001 (2010)

174. T. Friedmann, No radial excitations in low energy QCD (I): Diquarks and classification of mesons, Eur. Phys. J. C 73(2), 2298 (2013)

175. W. Erni, et al. (PANDA Collaboration), Physics performance report for PANDA: Strong interaction studies with antiprotons, arXiv: 0903.3905 [hep-ex] (2009) 